\title{
Integrating central extensions of Lie algebras via Lie 2-groups
}

\author{
Christoph Wockel Chenchang Zhu
}

\begin{abstract}
The purpose of this paper is to show how central extensions of (possibly infinitedimensional) Lie algebras integrate to central extensions of étale Lie 2-groups in the sense of [Get09, Hen08]. In finite dimensions, central extensions of Lie algebras integrate to central extensions of Lie groups, a fact which is due to the vanishing of $\pi_{2}$ for each finite-dimensional Lie group. This fact was used by Cartan (in a slightly other guise) to construct the simply connected Lie group associated to each finitedimensional Lie algebra.

In infinite dimensions, there is an obstruction for a central extension of Lie algebras to integrate to a central extension of Lie groups. This obstruction comes from non-trivial $\pi_{2}$ for general Lie groups. We show that this obstruction may be overcome by integrating central extensions of Lie algebras not to Lie groups but to central extensions of étale Lie 2-groups. As an application, we obtain a generalization of Lie's Third Theorem to infinite-dimensional Lie algebras.
\end{abstract}

Keywords. Infinite-dimensional Lie group, central extension, smooth group cohomology, group stack, Lie 2-group, integration of cocycles, Lie's Third Theorem, 2connected cover

\section{Contents}

1 Introduction 2

2 Differentiable hypercohomology and its geometric correspondence 6

2.1 Differentiable hypercohomology . . . . . . . . . . . . 6

2.2 From differentiable hypercohomology to Lie 2-groups . . . . . . . . . . 12

2.3 Cohomologous cocycles . . . . . . . . . . . . . 21

C. Wockel: Fachbereich Mathematik, Universität Hamburg (Germany); e-mail: christoph@ wockel.eu C. Zhu: Mathematisches Institut, Universität Göttingen (Germany); e-mail: chenchang.zhu@gmail.com

Mathematics Subject Classification (2010): Primary 22E65; Secondary 58H05, 58B25, 55N20 
3 Geometric cocycle constructions 22

3.1 Locally smooth cocycles . . . . . . . . . . . . . . . . 22

3.2 Differentiable cocycles . . . . . . . . . . . . . . . . . 24

3.3 Dependency on choices . . . . . . . . . . . . . . 30

3.4 A bundle-theoretic interpretation of the main construction . . . . . . . 32

4 Lie's Third Theorem $\quad 34$

4.1 Deriving Lie algebras from étale Lie 2-groups . . . . . . . . . . . . . . 34

4.2 Lie's Third Theorem for locally exponential Lie algebras . . . . . . . . . 38

5 An interpretation in terms of 2-connected covers 41

A Appendix: Differential calculus on locally convex spaces 43

B Appendix: Lie groupoids and the submersion Grothendieck pretopology on locally convex manifolds $\quad 47$

\section{Introduction}

Central extensions of Lie algebras and their integrability are closely related to Lie's Third Theorem. In fact, one can use the integration theory of central extensions of Lie algebras [Nee02] to decide (under some mild requirements) whether a given Lie algebra is the Lie algebra of a Lie group. In finite dimensions, this is always the case due to the vanishing of $\pi_{2}$ for each finite-dimensional Lie group. In infinite dimensions, $\pi_{2}$ does not always vanish and leads to Lie algebras which do not integrate to Lie groups [EK64, DL66]. A similar phenomenon occurs when integrating finite-dimensional Lie algebroids to Lie groupoids [Pra68, CF03]. In this case it is $\pi_{2}$ of the leaves that restricts the integrability of a Lie algebroid.

On the other hand, the theory of higher Lie group(oid)s has been much developed recently. Already in the early twentieth century, 2-groups were studied by Whitehead and his followers under various terms, such as crossed modules. They are also studied from the perspective of "gr-champ" (i.e. stacky groups) by Breen [Bre90]. More recently, various versions of 2-groups, with different strictness assumptions, have been studied as models for the string group [BCSS07, SP11] using a method of categorification, initiated by Baez' school [BL04]. However to treat all higher group(oid)s in various categories all together, the most efficient method is to apply Duskin and Glenn's idea of Kan complexes [Dus79, Gle82]. This method has the advantage that it easily gives the concept of Lie $n$-group(oid)s for all $n \in \mathbb{N}$ if we take our category to be the one of manifolds, or the concept of topological $n$-group(oid)s for all $n \in \mathbb{N}$ if we change our category to 
be the one of topological spaces (see e.g. [Hen08, Zhu09] for such a treatment and the additional requirement of the taken categories). This allows us to treat many theories uniformly without repeating proofs. Moreover, starting from Getzler's work on integration of nilpotent $L_{\infty}$-algebras [Get09], this sort of Lie $n$-group(oid)s have been widely used in many integration problems, for example the integration of general $L_{\infty}$-algebras of Henriques [Hen08], the integration of finite dimensional Lie algebroid [TZ06], the integration of Courant algebroid [LBv11, MT10, SZ11]. Thus people have a general belief that these Lie $n$-group(oid)s are the correct objects of a certain (higher) category that corresponds, via integration, to the one of various infinitesimal objects, for example, $L_{\infty}$-algebras, $L_{\infty}$ algebroids.

Using this sort of Lie 2-groups, we study in this article the integration of another sort of infinitesimal object, namely infinite-dimensional Lie algebras and their central extensions. We obtain a version of Lie's Third Theorem asserting that each locally exponential Lie algebra (see Definition A.4) with topologically split center integrates to an étale Lie 2 -group. The same question was studied in [Woc11a] by a completely different and less powerful concept of Lie 2-group, since it only admits a notion of smoothness "near the identity". Note that this concept of "locally smooth" Lie 2-group is only known to be equivalent to the one mentioned above in very special cases [WW13] that do not govern the situation we have in this paper. The value of the current article is that it extends the result of [Woc11a] to a global one. One can do this because we weaken (in a certain sense) the category of "smooth 2-spaces" from [Woc11a], which is nothing but the category of Lie groupoids with strict morphisms, to the bicategory of smooth stacks which is equivalent to the one of Lie groupoids with generalized morphisms and 2-morphisms. To obtain our result we then have enhanced the approach from [Woc11a] significantly because generalized morphisms are more involved than the strict ones. This effort is eventually rewarded by obtaining a globally smooth object integrating an infinite-dimensional Lie algebra.

We now go into some more detail. The basic idea behind the integration processes from [Nee02] (and [CF03]) is to integrate prescribed curvature 2-forms along certain triangles (respectively homotopies between paths). Suppose that $\mathfrak{g}$ is the Lie algebra of a simply connected Lie group $G$ and that $\omega: \mathfrak{g} \times \mathfrak{g} \rightarrow \mathfrak{z}$ is a continuous Lie algebra cocycle. If the period homomorphism $\pi_{2}(G) \stackrel{\text { per }_{\omega}}{\longrightarrow} \mathfrak{z}$ (see (1.1)) has discrete image, then $\omega$ has an integrating cocycle in the locally smooth Lie group cohomology $H_{\text {loc }}^{2}\left(G, \mathfrak{z} / \operatorname{per}_{\omega}\left(\pi_{2}(G)\right)\right)$, i.e., the differentiation homomorphism

$$
D: H_{\mathrm{loc}}^{2}\left(G, \mathfrak{z} / \operatorname{per}_{\omega}\left(\pi_{2}(G)\right)\right) \rightarrow H_{c}^{2}(\mathfrak{g}, \mathfrak{z})
$$

has the class of $\omega$ in its range [Nee02, Corollary 6.3]. This is shown by integrating $\omega$ along some carefully chosen triangles, an idea which dates back to van Est [Est58]. The 
procedure then reveals the obstruction against integration as a cocycle condition, which may also be viewed as an associativity constraint for enlarging a local group to a global one (cf. [Smi51a, Smi51b, Est62a, Est62b]). So one is naturally pushed to non-associative structures when searching for a general solution of the integration problem. Our Lie 2groups are such structures, which provide at the same time the next higher coherence that the problem naturally has (cf. the discussion in [Woc11a, Section 2]).

In this paper, we deal with the case when $\operatorname{per}_{\omega}\left(\pi_{2}(G)\right)$ is not discrete. In this case, $\mathfrak{z} / \operatorname{per}_{\omega}\left(\pi_{2}(G)\right)$ does not exist as a Lie group any more. One of the natural substitutes for it is the Lie 2-group $\left[\pi_{2}(G) \stackrel{\text { per }_{\omega}}{\longrightarrow} \mathfrak{z}\right]^{3}$ (see Example 2.11), which exists regardless of the discreteness of $\operatorname{per}_{\omega}\left(\pi_{2}(G)\right)$. However, if $\operatorname{per}_{\omega}\left(\pi_{2}(G)\right)$ is discrete, then $\mathfrak{z} / \operatorname{per}_{\omega}\left(\pi_{2}(G)\right)$ is equivalent to a direct factor of $\left[\pi_{2}(G) \stackrel{\text { per }_{\omega}}{\longrightarrow} \mathfrak{z}\right]$ and thus $\left[\pi_{2}(G) \stackrel{\operatorname{per}_{\omega}}{\longrightarrow} \mathfrak{z}\right]$ is the universal object taking over the rôle of $\mathfrak{z} / \operatorname{per}_{\omega}\left(\pi_{2}(G)\right)$. Moreover, extensions of $G$ by $\left[\pi_{2}(G) \stackrel{\text { per }_{\omega}}{\longrightarrow} \mathfrak{z}\right]$ live in the category of Lie 2-groups and thus permit us to incorporate the non-associativity that we mentioned above. In this sense our treatment is a natural extension of the procedure from [Nee02]. The price to pay for this freedom is that one has to work with group objects in smooth stacks (aka Lie 2-groups ${ }^{4}$ ) instead of group objects in smooth manifolds (aka Lie groups). This is technically more challenging but has similar underlying ideas.

Section 2 is concerned with setting up the theory of central extensions of Lie groups by abelian Lie 2-groups $[A \stackrel{\mu}{\rightarrow} B]$, in particular to show how such extensions can be obtained from certain Čech cohomology classes. This section builds heavily on [SP11, Section 3]. It provides the conceptual background for understanding the constructions in the next section.

Section 3 then presents the refinement of the aforementioned idea of integration along triangles. The problem that one has to overcome is that the cocycle condition that one has in $\mathfrak{z} / \operatorname{per}_{\omega}\left(\pi_{2}(G)\right)$ makes many arguments implicitly work. One example for this is the smoothness of the multiplication of the central extension of Lie groups associated to an integrating cocycle for $\omega$ as discussed in Section 3.4. All these implications now have to be built into the choices of the triangles, and this is the key point of Section 3. In a certain sense, the essence of this construction is subsumed in Figures 1 and 2.

Section 4 then provides the differentiation process which justifies naming the construction of the previous section "integration". We restrict in this treatment to étale Lie 2-groups, for which the differentiation leads to ordinary Lie algebras. The main result on this is the following

Theorem. If $G$ is a simply connected Lie group with Lie algebra $\mathfrak{g}$, $\mathfrak{z}$ is a Mackey-complete

\footnotetext{
${ }^{3}$ Another substitute would be the diffeological group $\mathfrak{z} / \operatorname{per}_{\omega}\left(\pi_{2}(G)\right)$, see [Woc11a, Remark 7.1]

${ }^{4}$ Notice that when $n=2$, Lie $n$-groups described via Kan complexes that we mentioned earlier are proven [Zhu09] to be equivalent to group objects in smooth stacks.
} 
locally convex space and $\omega: \mathfrak{g} \times \mathfrak{g} \rightarrow \mathfrak{z}$ is a continuous Lie algebra cocycle. Then the differentiation homomorphism

$$
D: \operatorname{Ext}\left(\underline{G},\left[\pi_{2}(G) \stackrel{\text { per }_{\omega}}{\longrightarrow} \mathfrak{z}\right]\right) \rightarrow \operatorname{Ext}(\mathfrak{g}, \mathfrak{z}) \cong H_{c}^{2}(\mathfrak{g}, \mathfrak{z})
$$

has $[\omega]$ in its image. Here $\underline{G}$ is the Lie group G viewed as a Lie 2-group (see Example 2.11).

This then implies readily the following generalization of Lie's Third Theorem that our construction allows for.

Theorem. If $\mathfrak{g}$ is a locally convex locally exponential Lie algebra such that $\mathfrak{z}:=\mathfrak{z}(\mathfrak{g}) \subseteq \mathfrak{g}$ is a complemented and Mackey-complete subspace, then there exists an étale Lie 2-group $G$ with $L(G) \cong \mathfrak{g}$.

In the end, we provide some background on infinite-dimensional manifolds and their derived concepts of Lie groups, Lie groupoids and smooth stacks.

\section{Conventions}

Unless stated otherwise, $G$ denotes throughout a 1-connected Lie group, modeled on a locally convex space, and $\mathfrak{g}$ denotes its Lie algebra. Moreover, $\mathfrak{z}$ stands for a Mackeycomplete locally convex vector space ${ }^{5}, \Gamma \subseteq \mathfrak{z}$ is a discrete subgroup so that $Z:=\mathfrak{z} / \Gamma$ is a Lie group with universal covering morphism $q: \mathfrak{z} \rightarrow Z$. In addition, $\omega: \mathfrak{g} \times \mathfrak{g} \rightarrow \mathfrak{z}$ will always denote a continuous Lie algebra cocycle. Associated to this data is the period homomorphism

$$
\operatorname{per}_{\omega}: \pi_{2}(G) \rightarrow \mathfrak{z}, \quad[\sigma] \mapsto \int_{\sigma} \omega^{l},
$$

where $\omega^{l}$ is the left-invariant 2-form on $G$ with $\omega^{l}(e)=\omega$ (cf. [Nee02, Section 5]). Note that under our assumptions $\pi_{2}(G) \cong H_{2}(G)$ by the Hurewicz homomorphism and we identify $\pi_{2}(G)$ with $H_{2}(G)$ throughout.

We denote by $\Delta^{(n)} \subseteq \mathbb{R}^{n}$ the standard $n$-simplex, viewed as a manifold with corners. By $C^{\infty}\left(\Delta^{(n)}, G\right)$ we mean the manifold of smooth $n$-simplices in $G$ (see also Proposition A.6) and by $C_{*}^{\infty}\left(\Delta^{(n)}, G\right)$ those smooth $n$-simplices that are base-point preserving maps, where the base-point of $\Delta^{(n)}$ is 0 and the base-point of $G$ is the identity. For a simplicial complex $\Sigma$ we will denote by $C_{p w}^{\infty}(\Sigma, G)$ the piece-wise smooth maps (cf. Remark A.7). The simplicial manifold that shall play an important rôle in this paper is the classifying simplicial space $\mathbf{B} G_{\bullet}=\left(G^{i}\right)_{i \in \mathbb{N}_{0}}$ (with the product smooth structure and the convention $G^{0}:=*$ ) and the standard simplicial maps $p_{i}^{(n)}: \mathbf{B} G^{(n)} \rightarrow \mathbf{B} G^{(n-1)}$ (cf. Example 2.1). Moreover, $\mu: A \rightarrow B$ is always a morphism between the abelian Lie groups $A$ and $B$.

\footnotetext{
${ }^{5}$ Mackey-complete locally convex spaces also run under the name convenient vector space, in particular each complete locally convex space is of this type, cf. [KM97, Theorem I.2.14]
} 


\section{Differentiable hypercohomology and its geometric cor- respondence}

\subsection{Differentiable hypercohomology}

The hypercohomology of complexes of sheaves on manifolds, action groupoids and complex stacks is explicitly studied for instance in [Bry93, Gom05] and [FHRZ08, §A.2]. Here we extend it to the category of simplicial manifolds and relate it to our construction using a suitable covering constructed in Section 3. We emphasize the Čech approach to differentiable hypercohomology and we are mostly interested in the simplicial manifold B $G_{\bullet}$ (i.e., the nerve of the Lie groupoid $G \rightrightarrows *$, see Example 2.1).

Recall that a simplicial manifold $X_{\bullet}$ is a functor $\Delta^{\mathrm{op}} \rightarrow$ Man, where $\Delta$ is the standard simplex category of finite ordinal numbers $[n]$ and non-decreasing maps $[n] \rightarrow[m]$. This has the alternative description as a collection of manifolds $X_{n}$ and structure maps

$d_{k}^{n}: X_{n} \rightarrow X_{n-1}$ (face maps) $s_{k}^{n}: X_{n} \rightarrow X_{n+1}$ (degeneracy maps), $k \in\{0,1,2, \ldots, n\}$

that satisfy the usual coherence conditions (see for instance [GJ99, Chapter I.1]).

Example 2.1. $\quad$ a) We can interpret each manifold as constant simplicial space with $X_{n}=M$ for all $n$ and all structure maps to be the identity.

b) Given a Lie groupoid $\mathcal{G}^{6}:=\left(G_{1} \underset{\mathrm{t}}{\stackrel{\mathrm{s}}{\rightrightarrows}} G_{0}\right)$, we complete it to a simplicial manifold BG., with

$$
\mathbf{B} \mathcal{G}_{n}=G_{1} \times_{\mathbf{s}, G_{0}, \mathbf{t}} G_{1} \times_{\mathbf{s}, G_{0}, \mathbf{t}} \cdots \times_{\mathbf{s}, G_{0}, \mathbf{t}} G_{1}, \quad\left(n \text { copies of } G_{1}\right),
$$

for $n \geq 1$ and $\mathbf{B} G_{0}=G_{0}$. The face maps, for $n \geq 2$, are given by

$$
d_{k}^{n}\left(g_{1}, \ldots, g_{n}\right)= \begin{cases}\left(g_{2}, \ldots, g_{n}\right) & k=0 \\ \left(g_{1}, \ldots, g_{k} g_{k+1}, \ldots, g_{n}\right) & 0<k<n \\ \left(g_{1}, \ldots, g_{n-1}\right) & k=n\end{cases}
$$

and the degeneracy maps, for $n \geq 1$, by

$$
s_{k}^{n}\left(g_{1}, \ldots, g_{n}\right)= \begin{cases}\left(1_{\mathbf{t}\left(g_{1}\right)}, g_{1} \ldots, g_{n}\right) & k=0 \\ \left(g_{1}, \ldots, g_{k}, 1_{\mathbf{t}\left(g_{k+1}\right)}, g_{k+1}, \ldots, g_{n}\right) & 0<k<n \\ \left(g_{1}, \ldots, g_{n}, 1_{\mathbf{s}\left(g_{n}\right)}\right) & k=n .\end{cases}
$$

Moreover, $d_{0}^{1}(g)=\mathbf{s}(g), d_{1}^{1}(g)=\mathbf{t}(g), s_{0}^{0}(x)=e(x)$, where $e: G_{0} \rightarrow G_{1}$ is the identity embedding. This construction is known as the nerve of the Lie groupoid

\footnotetext{
${ }^{6}$ For a Lie groupoid we require source and target map to be surjective submersions in the sense of Appendix B
} 
$\mathcal{G}$. We call it $\mathbf{B} \mathcal{G}$. because its geometric realization is the classifying space of $\mathcal{G}$ [Seg68]. If $\mathcal{G}=(G \rightrightarrows *)$, then we also denote $\mathbf{B} \mathcal{G} \bullet$ by $\mathbf{B} G_{\bullet}$.

For a simplicial manifold $X_{\bullet}$, a sheaf $\mathcal{F}^{\bullet}$ on $X_{\bullet}$ consists of sheaves $\mathcal{F}^{n}$ on $X_{n}$ for all $n$ and morphisms $F^{\bullet}(\alpha): X_{\bullet}(\alpha)^{*} \mathcal{F}^{n} \rightarrow \mathcal{F}^{m}$ for each $\alpha:[n] \rightarrow[m]$ such that $F^{\bullet}(\alpha \circ \beta)=$ $F^{\bullet}(\alpha) \circ F^{\bullet}(\beta)$ [Del74, $\left.\S 5.1 .6\right]$. Alternatively these morphisms can also be described by morphisms $D_{k}^{n}:\left(d_{k}^{n}\right)^{*} \mathcal{F}^{n-1} \rightarrow \mathcal{F}^{n}$ and $S_{k}^{n}:\left(s_{k}^{n}\right)^{*} \mathcal{F}^{n+1} \rightarrow \mathcal{F}^{n}$ satisfying the corresponding compatibility conditions. Likewise, we define morphisms of sheaves as in [Del74, $\S 5.1 .6]$. This then leads to the notion of a (bounded below) complex of sheaves on $X$.

$$
\mathcal{F}_{*}^{\bullet}=\left(\left(\mathcal{F}_{n}^{\bullet}\right)_{n \in \mathbb{N}_{0}}, \mathcal{F}_{n}^{\bullet} \stackrel{d_{n}}{\longrightarrow} \mathcal{F}_{n+1}^{\bullet}\right)
$$

(see also [Gom05, §3.2]).

A covering $\mathcal{U}$ of a simplicial manifold $X$. consists of a simplicial set $I^{\bullet}$ and a covering $\left(U_{i}^{(n)}\right)_{i \in I^{(n)}}$ of $X_{n}$ such that $X_{\bullet}(\alpha)\left(U_{i}^{(m)}\right) \subseteq U_{I^{\bullet}(\alpha)(i)}^{(n)}$ for each $\alpha:[n] \rightarrow[m]$. One can demand less structure for a covering of a simplicial space, see [SP11] or [WW13]. We demand all this structure for making the normalization arguments later on work. In particular, a covering induces another simplicial space $\mathcal{U}_{\bullet}$ with $\mathcal{U}_{n}:=\coprod_{i \in I^{n}} U_{i}^{(n)}$ and the inclusions induce a simplicial map $\mathcal{U}_{\bullet} \rightarrow X_{\bullet}$. The following lemma shows that one can always extend coverings of $X_{n}$ to coverings of $X_{\bullet}$.

Lemma 2.2. If $X_{\bullet}$ is a simplicial manifold and $\left(U_{j}\right)_{j \in J}$ is a covering of $X_{m}$, then there exists a covering $\mathcal{U}$ of $X_{\bullet}$ such that $\left(U_{i \in I^{(m)}}^{(m)}\right)$ is a refinement of $\left(U_{j}\right)_{j \in J}$.

Proof. We denote $\Delta(m, n):=\operatorname{Hom}_{\Delta}([m],[n])$ (note that this is a finite set). We first observe that $J$ determines a simplicial set $I^{\bullet}$ with $I^{(n)}:=J^{\Delta(m, n)}$ and with $\alpha:[n] \rightarrow\left[n^{\prime}\right]$ getting mapped to

$$
\alpha^{\Delta}: J^{\Delta\left(m, n^{\prime}\right)} \rightarrow J^{\Delta(m, n)}, \quad \alpha^{\Delta}\left(\left(j_{f}\right)_{f \in \Delta\left(m, n^{\prime}\right)}\right)_{g}=j_{\alpha \circ g}
$$

Now for each $f \in \Delta(m, n)$ we pull back the covering $\left(U_{j}\right)_{j \in J}$ of $X_{m}$ to a covering of $X_{n}$ and take as a covering of $X_{n}$ the coarsest common refinement $\left(U_{i}^{(n)}\right)_{i \in I^{(n)}}$ with

$$
U_{i}^{(n)}:=X_{\bullet}\left(f_{0}\right)^{-1}\left(U_{j_{f_{0}}}\right) \cap \ldots \cap X_{\bullet}\left(f_{s}\right)^{-1}\left(U_{j_{f_{s}}}\right)
$$

(where $i=\left(j_{f_{0}}, \ldots, j_{f_{s}}\right)$ with $\left.s=|\Delta(m, n)|\right)$ of all such coverings. To check that this is indeed a covering we have to show that for each $\alpha:\left[n^{\prime}\right] \rightarrow[n]$ and each $i=\left(j_{f}\right)_{f \in \Delta([m],[n])}$ we have

$$
\begin{aligned}
& X_{\bullet}(\alpha)\left(U_{i}^{(n)}\right) \subseteq U_{\alpha^{\Delta}(i)}^{\left(n^{\prime}\right)} \\
\Leftrightarrow & X_{\bullet}(\alpha)\left(X_{\bullet}\left(f_{0}\right)^{-1}\left(U_{j_{f_{0}}}\right) \cap \cdots \cap X_{\bullet}\left(f_{s}\right)^{-1}\left(U_{j_{f_{s}}}\right)\right) \subseteq X_{\bullet}(f)^{-1}\left(U_{j_{\alpha \circ f}}\right) \forall f \in \Delta\left(m, n^{\prime}\right) \\
\Leftrightarrow & X_{\bullet}(\alpha \circ f)\left(X_{\bullet}\left(f_{0}\right)^{-1}\left(U_{j_{f_{0}}}\right) \cap \cdots \cap X_{\bullet}\left(f_{s}\right)^{-1}\left(U_{j_{f_{s}}}\right)\right) \subseteq U_{j_{\alpha \circ f}} \forall f \in \Delta\left(m, n^{\prime}\right) .
\end{aligned}
$$


The latter is true since for each $f \in \Delta\left(m, n^{\prime}\right)$ we have that $\alpha \circ f=f_{i}$ for some $i$. To complete the proof we observe that the canonical map $I^{(m)} \rightarrow J,\left(j_{f}\right)_{f \in \Delta(m, m)} \mapsto j_{\operatorname{id}_{[m]}}$ induces the corresponding refinement.

Let now $\mathcal{U}$ be a covering of $X_{\bullet}$. Then we set

$$
\check{C}^{p, q, r}:=\prod_{i_{0}, \ldots, i_{q} \in I^{(p)}} \mathcal{F}_{r}^{p}\left(U_{i_{0}}^{(p)} \cap \ldots \cap U_{i_{q}}^{(p)}\right) .
$$

We have $\delta_{\mathrm{gp}}\left(:=d_{1,0,0}\right): \check{C}^{p-1, q, r} \rightarrow \check{C}^{p, q, r}$ defined by

$$
\left(\delta_{\mathrm{gp}} f\right)_{i_{0}, \ldots, i_{q}}=\sum_{k=0}^{p}(-1)^{k+p} D_{k}^{p}(\underbrace{f_{d_{k}^{p}\left(i_{0}\right), \ldots, d_{k}^{p}\left(i_{q}\right)} \circ d_{k}^{p}}_{\in\left(d_{k}^{p}\right)^{*} \mathcal{F}^{p-1}})
$$

and $\check{\delta}\left(:=d_{0,1,0}\right): \check{C}^{p, q, r} \rightarrow \check{C}^{p, q+1, r}$ defined by the $\check{C}$ ech differential

$$
(\check{\delta} f)_{i_{0}, \ldots, i_{q+1}}(x)=\sum_{l=0}^{q+1}(-1)^{l} f_{i_{0}, \ldots, \widehat{i}_{l}, \ldots, i_{q+1}}(x) .
$$

There is another differential $d\left(:=d_{0,0,1}\right): \check{C}^{p, q, r} \rightarrow \check{C}^{p, q, r+1}$, induced by the differential $d_{r}: \mathcal{F}_{r} \rightarrow \mathcal{F}_{r+1}$ in the sheaf complex. Then $\left(\check{C}^{p, q, r}, \delta_{\mathrm{gp}}, \check{\delta}, d\right)$ is a triple complex and the total complex is $\check{C}^{N}:=\oplus_{N=p+q+r} \check{C}^{p, q, r}$ with the total differential $D_{3}=$ $\delta_{\mathrm{gp}}+(-1)^{p} \check{\delta}+(-1)^{p+q} d$.

Definition 2.3. The Čech hypercohomology $\check{H}_{\mathcal{U}}^{n}\left(X_{\bullet}, \mathcal{F}_{*}^{\bullet}\right)$ of the complex of sheaves $\mathcal{F}_{*}^{\bullet}$ on $X$. with respect to the covering $\mathcal{U}$ is the cohomology of the total complex of $\left(\check{C}^{p, q, r}, \delta_{\mathrm{gp}}, \check{\delta}, d\right)$. The group of $n$-cocycles of this triple complex is denoted $\check{Z}_{\mathcal{U}}^{n}\left(X_{\bullet}, \mathcal{F}_{*}^{\bullet}\right)$ and its elements are called differentiable cocycles.

On the other hand, one can define sheaf hypercohomology $H_{s h}^{n}\left(X_{\bullet}, \mathcal{F}_{*}^{\bullet}\right)$ to be the hyper derived functor of the functor

$$
\mathcal{F}_{*}^{\bullet} \mapsto H^{0}\left(\Gamma\left(\mathcal{F}_{*}^{\bullet}\right)\right)
$$

where the section functor $\Gamma$ for a sheaf $\mathcal{F}^{\bullet}$ on $X$ • is given by $\mathcal{F}^{\bullet} \mapsto \operatorname{ker}\left(D_{0}-D_{1}: \mathcal{F}^{0}\left(X_{0}\right) \rightarrow \mathcal{F}^{1}\left(X_{1}\right)\right)$. The hyper derived functor is then given by the usual construction using injective resolutions as in [Fri82, Chapter 2], see also [Del74, Con03]. The following proposition generalizes the case of the relation between Čech cohomology and sheaf cohomology. Recall that a covering $\mathcal{U}$ of $X$ • is called $\mathcal{F}$-acyclic if $H^{\geq 1}\left(U_{i_{0}}^{(n)} \cap \ldots \cap U_{i_{q}}^{(n)}, \mathcal{F}_{r}^{n}\right)=0$ for all finite subsets $\left\{i_{0}, \ldots, i_{q}\right\} \subseteq I^{(n)}$ and all $n, r$. The reasoning of [FHRZ08, $\S$ A.2] then carries over to show 
Proposition 2.4. In the above setup, there is a morphism of abelian groups

$$
\check{H}_{\mathcal{U}}^{n}\left(X_{\bullet}, \mathcal{F}_{*}^{\bullet}\right) \rightarrow H_{s h}^{n}\left(X_{\bullet}, \mathcal{F}_{*}^{\bullet}\right)
$$

In particular, if $\mathcal{U}$ is an $\mathcal{F}_{r}$-acyclic covering of $\mathcal{X}$ for each $r$, then $\check{H}_{\mathcal{U}}^{n}\left(\mathcal{X}, \mathcal{F}_{*}^{\bullet}\right) \cong H_{s h}^{n}\left(\mathcal{X}, \mathcal{F}_{*}^{\bullet}\right)$.

Thus the Čech hypercohomology of $\mathcal{F}_{*}^{\bullet}$, defined as the direct limit

$$
\check{H}^{n}\left(X_{\bullet}, \mathcal{F}_{*}^{\bullet}\right):=\lim _{\longrightarrow} \check{H}_{\mathcal{U}}^{n}\left(X_{\bullet}, \mathcal{F}_{*}^{\bullet}\right)
$$

is isomorphic to the sheaf cohomology $H_{s h}^{n}\left(X_{\bullet}, \mathcal{F}_{*}^{\bullet}\right)$ if each covering admits an $\mathcal{F}$-acyclic refinement. When the existence of acyclic coverings is not guaranteed, one needs to take the limit over all hyper-coverings but not only coverings of $X_{\bullet}$ as explained in [Fri82]. Then the same result holds. However, each covering is in particular a hyper-covering and all our constructions will yield cocycles on usual coverings. In addition, the equivalences of cocycles that we construct will also live on usual coverings. Thus our constructions will lead to well-defined classes in Čech cohomology and thus also in sheaf cohomology.

There is one additional condition on Čech cocycles, that simplifies computations a lot, which is the assumption that they are normalized. For this we consider

$$
\mathcal{F}_{r, 0}^{p}\left(U_{i_{0}}^{(p)} \cap \ldots \cap U_{i_{q}}^{(p)}\right):=\bigcap_{l=0}^{q} \operatorname{ker}\left(\left(\check{s}_{l}^{q}\right)^{*}\right),
$$

with

$\check{s}_{l}^{q}: U_{i_{0}}^{(p)} \cap \ldots \cap U_{i_{q}}^{(p)} \stackrel{\cong}{\rightarrow} U_{i_{0}}^{(p)} \cap \ldots \cap U_{i_{l}} \cap U_{i_{l}} \cap \ldots \cap U_{i_{q+1}}^{(p)}, \quad x_{i_{0}, \ldots, i_{q}} \mapsto x_{i_{0}, \ldots, i_{l-1}, i_{l}, i_{l}, i_{l+1}, \ldots, i_{q}}$

the standard Čech degeneracy maps and

$$
\check{C}_{0}^{p, q, r}:=\bigcap_{k=0}^{p-1} \operatorname{ker}\left(\sigma_{k}\right) \cap \prod_{i_{0}, \ldots, i_{q} \in I^{(p)}} \mathcal{F}_{r, 0}^{p}\left(U_{i_{0}}^{(p)} \cap \ldots \cap U_{i_{q}}^{(p)}\right)
$$

with

$$
\sigma_{k}: \check{C}^{p, q, r} \rightarrow \check{C}^{p-1, q, r}, \quad \sigma_{k}(f)_{i_{0}, \ldots, i_{q}}=S_{k}^{p-1}\left(f_{s_{k}^{p-1}\left(i_{0}\right), \ldots, s_{k}^{p-1}\left(i_{q}\right)} \circ s_{k}^{p-1}\right)
$$

the degeneracy map induced by the degeneracies $s_{k}^{p}: X_{p} \rightarrow X_{p+1}$ and $S_{k}^{p-1}:\left(s_{k}^{p-1}\right)^{*} \mathcal{F}^{p} \rightarrow \mathcal{F}^{p-1}$. It is clear that $\left(\check{C}_{0}^{p, q, r}, \delta_{\mathrm{gp}}, \check{\delta}, d\right)$ is a sub triple complex of $\left(\check{C}^{p, q, r}, \delta_{\mathrm{gp}}, \check{\delta}, d\right)$ and thus the cohomology of the associated total complex

$$
\check{H}_{0, \mathcal{U}}^{n}\left(X_{\bullet}, \mathcal{F}_{*}^{\bullet}\right)
$$

comes equipped with a natural morphism

$$
\check{H}_{0}^{n}\left(X_{\bullet}, \mathcal{F}_{*}^{\bullet}\right):=\lim _{\longrightarrow} \check{H}_{0, \mathcal{U}}^{n}\left(X_{\bullet}, \mathcal{F}_{*}^{\bullet}\right) \rightarrow \check{H}^{n}\left(X_{\bullet}, \mathcal{F}_{*}^{\bullet}\right)
$$


from the normalized to the ordinary Čech cohomology. The normalized Čech cohomology is what we will work with in this article. For its conceptual interpretation we will first show that it actually agrees with the non-normalized Čech cohomology and thus with sheaf cohomology in many interesting cases.

Proposition 2.5. Suppose that $\mathcal{A}=$

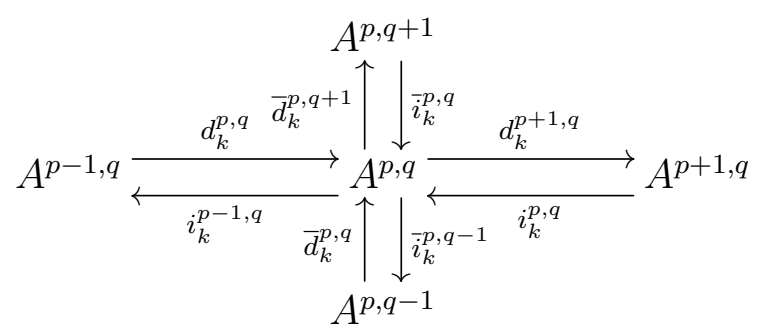

is a bi-cosimplicial abelian group. Then the normalized cochains

$$
A_{0}^{p, q}=\bigcap_{k=0}^{p-1} \operatorname{ker}\left(i_{k}^{p-1, q}\right) \cap \bigcap_{k=0}^{q-1} \operatorname{ker} \bar{i}_{k}^{p, q-1}
$$

form a sub bi-cosimplicial abelian group $\mathcal{A}_{0}$ and the inclusion of the associated total complexes $\operatorname{Tot}\left(\mathcal{A}_{0}\right) \rightarrow \operatorname{Tot}(\mathcal{A})$ induces an isomorphism in cohomology.

Proof. We first define the vertically normalized cochains $\mathcal{A}_{v}$ to be $A_{v}^{p, q}=\cap_{k=0}^{p-1} \operatorname{ker}\left(i_{k}^{p-1, q}\right)$, which is also a sub bi-cosimplicial abelian group of $\mathcal{A}$. Likewise, we define the horizontally normalized cochains $\mathcal{A}_{h}$ to be given by $A_{h}^{p, q}=\cap_{k=0}^{q-1} \operatorname{ker}\left(i_{k}^{p, q-1}\right)$. Observe that $\mathcal{A}_{0}=\left(\mathcal{A}_{v}\right)_{h}$. By the dual Dold-Kan correspondence [Wei94, Corollary 8.4.3] the cochain complex of a cosimplicial abelian group has the same cohomology as its normalized sub complex. Thus $\operatorname{Tot}\left(\mathcal{A}_{v}\right) \rightarrow \operatorname{Tot}(\mathcal{A})$ and $\operatorname{Tot}\left(\left(\mathcal{A}_{v}\right)_{h}\right) \rightarrow \operatorname{Tot}\left(\mathcal{A}_{v}\right)$ induce isomorphisms in cohomology.

Corollary 2.6. If $X_{\bullet}$ is a simplicial manifold and $\mathcal{F}_{*}^{\bullet}$ is a complex of sheaves on $X_{\bullet}$, then the canonical morphism

$$
\check{H}_{0}^{n}\left(X_{\bullet}, \mathcal{F}_{*}^{\bullet}\right) \rightarrow \check{H}^{n}\left(X_{\bullet}, \mathcal{F}_{*}^{\bullet}\right)
$$

is an isomorphism.

Remark 2.7. We spell out the definition of Čech hypercohomology from Definition 2.3 in the case that we are interested in for convenience and later reference. First note that for the constant simplicial manifold associated to a manifold $M$ (cf. Example 2.1) the Čech hypercohomology is what is also called the non-abelian cohomology $\check{H}^{2}(M, A \stackrel{\mu}{\rightarrow} B)$ with coefficients in the crossed module $\mu: A \rightarrow B$ (see for instance [NW11, Woc11b, BS07, Bar06, Bre94, Gir71, Ded60]). 
In this article we will mainly be interested in the simplicial manifold $\mathrm{B} G$ • the nerve of the infinite-dimensional Lie groupoid $G \rightrightarrows *^{7}$ with the 2-term complex of sheaves of germs of smooth functions with value in $A$ and $B$, where $A \stackrel{\mu}{\rightarrow} B$ is a morphism of abelian Lie groups.

Then our triple complex is

$\check{C}^{p, q,-1}=\prod_{i_{0}, \ldots, i_{q} \in I^{(p)}} C^{\infty}\left(U_{i_{0}}^{(p)} \cap \cdots \cap U_{i_{q}}^{(p)}, A\right) \quad$ and $\quad \check{C}^{p, q, 0}=\prod_{i_{0}, \ldots, i_{q} \in I^{(p)}} C^{\infty}\left(U_{i_{0}}^{(p)} \cap \cdots \cap U_{i_{q}}^{(p)}, B\right)$,

with $d=\mu_{*}$, i.e.,

$$
\left(\mu_{*} f\right)_{i_{0}, \ldots, i_{q}}(x)=\mu\left(f_{i_{0}, \ldots, i_{q}}(x)\right) .
$$

Then a 2-cocycle of the total differential in this triple complex is given by maps (we also write down the slightly more intuitive names of them that we will use in the geometric construction later on):

$$
\begin{aligned}
& \phi^{1,1,0}\left(=\gamma=\left(\gamma_{i, j}\right)\right): U_{i}^{(1)} \cap U_{j}^{(1)} \rightarrow B, \forall i, j \in I^{(1)} \\
& \phi^{1,2,-1}\left(=\eta=\left(\eta_{i, j, k}\right)\right): U_{i}^{(1)} \cap U_{j}^{(1)} \cap U_{k}^{(1)} \rightarrow A, \forall i, j, k \in I^{(1)} \\
& \phi^{2,0,0}\left(=F=\left(F_{i}\right)\right): U_{i}^{(2)} \rightarrow B, \forall i \in I^{(2)} \\
& \phi^{2,1,-1}\left(=\Phi=\left(\Phi_{i, j}\right)\right): U_{i}^{(2)} \cap U_{j}^{(2)} \rightarrow A, \forall i, j \in I^{(2)} \\
& \phi^{3,0,-1}\left(=\Theta=\left(\Theta_{i}\right)\right): U_{i}^{(3)} \rightarrow A, \forall i \in I^{(3)}
\end{aligned}
$$

such that (when $r=0$ )

$$
\begin{gathered}
d_{0,1,0}\left(\phi^{1,2,-1}\right)(=\check{\delta}(\eta))=0 \\
d_{1,0,0}\left(\phi^{1,2,-1}\right)+d_{0,1,0}\left(\phi^{2,1,-1}\right)\left(=\delta_{\mathrm{gp}}(\eta)+\check{\delta}(\Phi)\right)=0 \\
d_{1,0,0}\left(\phi^{2,1,-1}\right)-d_{0,1,0}\left(\phi^{3,0,-1}\right)\left(=\delta_{\mathrm{gp}}(\Phi)-\check{\delta}(\Theta)\right)=0 \\
d_{1,0,0}\left(\phi^{3,0,-1}\right)\left(=\delta_{\mathrm{gp}}(\Theta)\right)=0
\end{gathered}
$$

and (involving mixings of $r=0$ and $r=1$ )

$$
\begin{gathered}
-d_{0,1,0}\left(\phi^{1,1,0}\right)-d_{0,0,1}\left(\phi^{1,2,-1}\right)\left(=-\check{\delta}(\gamma)-\mu_{*}(\eta)\right)=0 \\
d_{1,0,0}\left(\phi^{1,1,0}\right)+d_{0,1,0}\left(\phi^{2,0,0}\right)-d_{0,0,1}\left(\phi^{2,1,-1}\right)\left(=\delta_{\mathrm{gp}}(\gamma)+\check{\delta}(F)-\mu_{*}(\Phi)\right)=0 \\
d_{1,0,0}\left(\phi^{2,0,0}\right)-d_{0,0,1}\left(\phi^{3,0,-1}\right)\left(=\delta_{\mathrm{gp}}(F)-\mu_{*}(\Theta)\right)=0
\end{gathered}
$$

Two differentiable 2-cocycles $\left(\phi^{1,1,0}, \phi^{1,2,-1}, \phi^{2,0,0}, \phi^{2,1,-1}, \phi^{3,0,-1}\right)$ and $\left(\widetilde{\phi}^{1,1,0}, \widetilde{\phi}^{1,2,-1}\right.$, $\left.\widetilde{\phi}^{2,0,0}, \widetilde{\phi}^{2,1,-1}, \widetilde{\phi}^{3,0,-1}\right)$ are called equivalent if they differ by a coboundary, that is, there

\footnotetext{
${ }^{7}$ If $\operatorname{dim}(G)<\infty$, then $\pi_{2}(G)=0$ and the integration procedure we consider here is covered by [Nee02].
} 
exists

$$
\begin{aligned}
& \psi^{1,0,0}\left(=\xi=\left(\xi_{i}\right)\right):\left(U^{\prime \prime}\right)_{i}^{(1)} \rightarrow B \\
& \psi^{1,1,-1}\left(=\rho=\left(\rho_{i, j}\right)\right):\left(U^{\prime \prime}\right)_{i}^{(1)} \cap\left(U^{\prime \prime}\right)_{j}^{(1)} \rightarrow A \\
& \psi^{2,0,-1}\left(=\sigma=\left(\sigma_{i}\right):\left(U^{\prime \prime}\right)_{i}^{(2)} \rightarrow A\right.
\end{aligned}
$$

such that

$$
\begin{aligned}
-d_{0,1,0}\left(\psi^{1,1,-1}\right) & =\phi^{1,2,-1}-\widetilde{\phi}^{1,2,-1}\left(=-\check{\delta}(\rho)=\eta-\eta^{\prime}\right) \\
d_{1,0,0}\left(\psi^{1,1,-1}\right)+d_{0,1,0}\left(\psi^{2,0,-1}\right) & =\phi^{2,1,-1}-\widetilde{\phi}^{2,1,-1}\left(=\delta_{\mathrm{gp}}(\rho)+\check{\delta}(\sigma)=\Phi-\Phi^{\prime}\right) \\
d_{1,0,0}\left(\psi^{2,0,-1}\right) & =\phi^{3,0,-1}-\widetilde{\phi}^{3,0,-1}\left(=\delta_{\mathrm{gp}}(\sigma)=\Theta-\Theta^{\prime}\right) \\
-d_{0,1,0}\left(\psi^{1,0,0}\right)+d_{0,0,1}\left(\psi^{1,1,-1}\right) & =\phi^{1,1,0}-\widetilde{\phi}^{1,1,0}\left(=-\check{\delta}(\xi)+\mu_{*}(\rho)=\gamma-\gamma^{\prime}\right) \\
d_{1,0,0}\left(\psi^{1,0,0}\right)+d_{0,0,1}\left(\psi^{2,0,-1}\right) & =\phi^{2,0,0}-\widetilde{\phi}^{2,0,0}\left(=\delta_{\mathrm{gp}}(\xi)+\mu_{*}(\sigma)=F-F^{\prime}\right)
\end{aligned}
$$

holds on a common refinement $\mathcal{U}^{\prime \prime}$ of the two simplicial covers $\left(U_{i}^{(n)}\right)_{i \in I^{(n)}}$ and $\left({U^{\prime}}_{i}^{(n)}\right)_{i \in\left(I^{\prime}\right)(n)}$.

\subsection{From differentiable hypercohomology to Lie 2-groups}

In this section we will describe how to construct Lie 2-groups form differentiable cocycles, similar to [SP11, Theorem 99].

We first introduce the concept of a group object in a bicategory and afterwards the corresponding notions of extensions and central extensions of Lie 2-groups. We will be brief on this, our main reference is [SP11].

Definition 2.8. Let C be a bicategory with finite products. A group object in C (or Cgroup, for brevity) consists of the following data:

- an object $G$ in C

- a list of 1-morphisms

$$
\begin{array}{ll}
m: G \times G \rightarrow G & \text { (the multiplication) } \\
u: * \rightarrow G & \text { (the unit) }
\end{array}
$$

such that

$$
\left(p r_{1}, m\right): G \times G \rightarrow G \times G
$$

is an equivalence in $\mathrm{C}$. 
- a list of invertible 2-morphisms

$$
\left.\left.\begin{array}{ll}
\begin{array}{l}
a \\
\ell: m \circ(m \times \mathrm{id}) \Rightarrow m \circ(\mathrm{id} \times m)
\end{array} & \text { (the associator }) \\
\ell: m \circ(u \times \mathrm{id}) \Rightarrow \mathrm{id} \\
r: m \circ(\mathrm{id} \times u) \Rightarrow \mathrm{id}
\end{array}\right\} \quad \text { (the left, resp. right unit constraint }\right)
$$

subject to the requirement that certain coherence conditions hold. A C-group is strict if all the 2-morphisms above are identity 2-morphisms.

A 1-morphism $G \rightarrow H$ of C-groups consists of a morphism $F: G \rightarrow H$ in $\mathrm{C}$ and invertible 2-morphisms $F_{2}: m_{H} \circ(F \times F) \Rightarrow F \circ m_{G}$ and $F_{0}: F \circ u_{G} \Rightarrow u_{H}$ satisfying the corresponding coherence conditions. Likewise, a 2-morphism between 1-morphisms of C-groups consists of a 2-morphism between the underlying 1-morphisms in C satisfying a certain coherence condition.

We refer to [SP11, Definition 41, 42, 43], [Blo08, §4.3] and [BL04, p. 37] (the latter in the case that $\mathrm{C}$ is actually a strict 2-category) for the various coherence conditions mentioned above.

Definition 2.9. Let sSt (respectively éSt) be the bicategory of (respectively étale) Lie groupoids, i.e., objects are (respectively étale) Lie groupoids, 1-morphisms are generalized morphisms and 2-morphisms are morphisms between generalized morphisms (see Appendix B for details). Then a group object in sSt is also called a Lie 2-group. The corresponding bicategory is denoted Lie2 - groups. A Lie 2-group is étale if it is further a group object in éSt.

Notice that our notion of a Lie 2-group is equivalent to the notion of Lie 2-group from [Get09, Hen08], defined by pointed simplicial manifolds satisfying Kan conditions $\operatorname{Kan}(n, j)$ for all $0 \leq j \leq n$ and $\operatorname{Kan!}(n, j)$ for all $0 \leq j \leq n \geq 3$. This has been proven in [Zhu09].

Definition 2.10. An abelian C-group in a bicategory $C$ with finite products is a group object $(G, m, u, a, l, r)$, together with an invertible 2-morphism $\beta: m \Rightarrow m \circ T$, where $T: G \times G \rightarrow G \times G$ is the canonical flip automorphism, such that the corresponding coherence conditions [SP11, Definition 47] are fulfilled.

A 1-morphism of abelian 2-groups consists of a 1-morphism of the underlying Cgroups making the diagram from [SP11, Definition 48] commute. A 2-homomorphism of abelian 2-groups consists of an arbitrary 2-morphism between 1-morphisms of abelian C-groups.

Example 2.11. a) If $G$ is an arbitrary Lie group, then the Lie groupoid with objects and morphisms equal to $G$ and structure maps equal to $\mathrm{id}_{G}$ gets a strict group object in sSt if we take the multiplication to be induced my the multiplication morphism 
on $G$ and the uni to be the inclusion of the unit element (and all 2-morphisms to be trivial). Since the inclusion Man $\rightarrow$ sSt preserves products this is just the image in sSt of the group object $G$ in Man. We will denote this Lie 2-group by $\underline{G}$.

b) If $\mu: A \rightarrow B$ is a morphism of abelian Lie groups, then we get a Lie groupoid $(A \times$ $B \rightrightarrows B)$ with $s(a, b)=b, t(a, b)=\mu(a) b, i_{b}=\left(e_{A}, b\right)$ and $\left(a^{\prime}, \mu(a) b\right) \circ(a, b)=$ $\left(a^{\prime} a, b\right)$. This inherits the structure of a strict group object in sSt from the group multiplication on $A \times B$ and $B$ (thus $m$ is a honest morphism of Lie groupoids), which is abelian (where we may choose $\beta$ to be the identity). We will denote this abelian Lie 2-group by $[A \stackrel{\mu}{\rightarrow} B]$.

Definition 2.12. An extension of $\underline{G}$ by $[A \stackrel{\mu}{\rightarrow} B]$ consists of

- a Lie 2-group $\widehat{G}$

- 1-morphisms $[A \stackrel{\mu}{\rightarrow} B] \stackrel{p}{\rightarrow} \widehat{G}$ and $\widehat{G} \stackrel{q}{\rightarrow} G$

such that their composition is equal ${ }^{8}$ to the canonical 1-morphism $[A \stackrel{\mu}{\rightarrow} B] \rightarrow * \rightarrow \underline{G}$ and that $\widehat{G} \rightarrow \underline{G}$ is a principal $[A \stackrel{\mu}{\rightarrow} B]$-2-bundle (cf. [SP11, NW11, Woc11b] $]^{9}$ ). Two extensions $[A \stackrel{\mu}{\rightarrow} B] \stackrel{p}{\rightarrow} \widehat{G} \stackrel{q}{\rightarrow} \underline{G}$ and $[A \stackrel{\mu}{\rightarrow} B] \stackrel{p^{\prime}}{\rightarrow} \widehat{G}^{\prime} \stackrel{q^{\prime}}{\rightarrow} \underline{G}$ are equivalent if there exist a 1-morphism $f: \widehat{G} \rightarrow \widehat{G}^{\prime}$ and a 2-morphisms $\lambda: f \circ p \Rightarrow p^{\prime}$

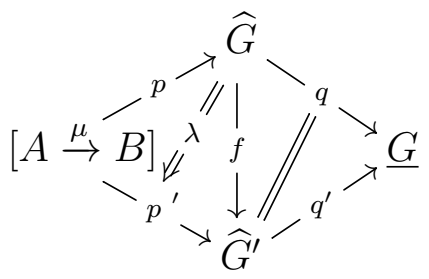

such that $q=q^{\prime} \circ f$. In this case we also call $(f, \lambda)$ an equivalence of central extensions.

Example 2.13. Suppose $G$ is a 1 -connected Lie group. The space

$$
P_{e} G:=\{\gamma \in C([0,1], G) \mid \gamma(0)=e\}
$$

of continuous pointed paths in $G$ is again a Lie group [GN14] with respect to the topology of uniform convergence and point-wise multiplication. Thus the evaluation map ev: $P_{e} G \rightarrow G$, $\gamma \mapsto \gamma(1)$ is a smooth group homomorphism and has a smooth section $\sigma: U \rightarrow P_{e} G$ on some identity neighborhood $U \subseteq G$. We extend this to a (in general non-continuous) section $\sigma: G \rightarrow P_{e} G$. It follows from the existence of a smooth local section that ev is a

\footnotetext{
${ }^{8}$ Note that equality is the only sensible thing here since $\underline{G}$ is a discrete groupoid.

${ }^{9}$ Unlike in [Woc11b], we do here allow arbitrary morphisms of smooth stacks as local trivializations, not only those which are represented by smooth functors, cf. [SP11, NW11].
} 
submersion [NSW13, Appendix B]. The kernel ker(ev) is the pointed (continuous) loop group $\Omega G$, which has the universal covering

$$
\pi_{2}(G) \rightarrow \widetilde{\Omega G} \rightarrow \Omega G
$$

Since continuous group automorphisms of $\Omega G$ lift in a unique way to group automorphisms of $\widetilde{\Omega G}$, we have that $P_{e} G$ acts by a lift of the conjugation action (from the right) on $\widetilde{\Omega G}$, which is smooth since $\pi_{2}(G)$ is discrete. Thus this action, along with the canonical map $\tau: \widetilde{\Omega G} \rightarrow P_{e} G$ is a smooth crossed module and thus determines a Lie 2-group $\Pi_{2}(G)$ [NSW13, Example 4.3], [Woc11b, Remark 2.4], [Por08, FB02, BS76]. It is of a quite simple nature, since the multiplication and inversion morphisms are represented by smooth functors on the action groupoid $\Gamma$ of the action of $\widetilde{\Omega G}$ on $P_{e} G$ induced by $\tau$.

Now $\Pi_{2}(G)$ comes along with a homomorphism of Lie 2-groups into $G$, induced by ev. Moreover, $\left[\pi_{2}(G) \rightarrow 0\right]$ embeds canonically into $\Pi_{2}(G)$ if we consider $\pi_{2}(G)$ as a subgroup of $\widetilde{\Omega G}$. We thus obtain a sequence of Lie 2 -groups

$$
\left[\pi_{2}(G) \rightarrow 0\right] \rightarrow \Pi_{2}(G) \rightarrow \underline{G} .
$$

That this is in particular an extension we have to check that $\Pi_{2}(G) \rightarrow \underline{G}$ is a principal 2-bundle. For this it suffices to observe that over $U_{g}:=g \cdot U$ we have the smooth section $\sigma_{g}(x)=\sigma(g) \cdot \sigma\left(g^{-1} \cdot x\right)$ of ev and that this induces a smooth functor

$$
\left[\pi_{2}(G) \rightarrow 0\right] \times\left.\underline{U_{g}} \rightarrow \Pi_{2}(G)\right|_{U_{g}}, \quad\left(a, \mathrm{id}_{x}\right) \mapsto\left(a, \sigma_{g}(x)\right) .
$$

The latter can easily be shown to be a weak equivalence.

Lemma 2.14. [SP11, Lemma 82] Let $[A \stackrel{\mu}{\rightarrow} B] \rightarrow \widehat{G} \rightarrow \underline{G}$ be an extension. Then there exists a 1-morphism $\underline{G} \rightarrow A u t([A \stackrel{\mu}{\rightarrow} B])$ of 2-groups ${ }^{10}$, unique up to unique 2-morphism.

Definition 2.15. An extension $[A \stackrel{\mu}{\rightarrow} B] \rightarrow \widehat{G} \rightarrow \underline{G}$ is central if the 1-morphism from the preceding Lemma is 2-equivalent to the trivial one.

Remark 2.16. If $A$ is a Lie group and $G$ a Lie group, then we define a Lie monoid extension of $G$ by $A$ to be a principal $A$-bundle $\widehat{G} \rightarrow G$ which is a Lie monoid such that $\widehat{G} \rightarrow G$ is a homomorphism of monoids, as well as the inclusion $A \rightarrow \widehat{G}, a \mapsto e_{\widehat{G}}$. $a$.

We will now see that $\widehat{G} \rightarrow G$ is already an extension of Lie groups, i.e., that $p r_{1} \times$ $m: \widehat{G} \times \widehat{G} \rightarrow \widehat{G} \times \widehat{G}$ is a diffeomorphism. In fact, consider the factorization of $\mathrm{pr} \times m_{\widehat{G}}=$

${ }^{10} 2$-groups are understood as group objects in the 2-category of categories. 
$p \circ \beta$ through the canonical maps to and from the pull-back:

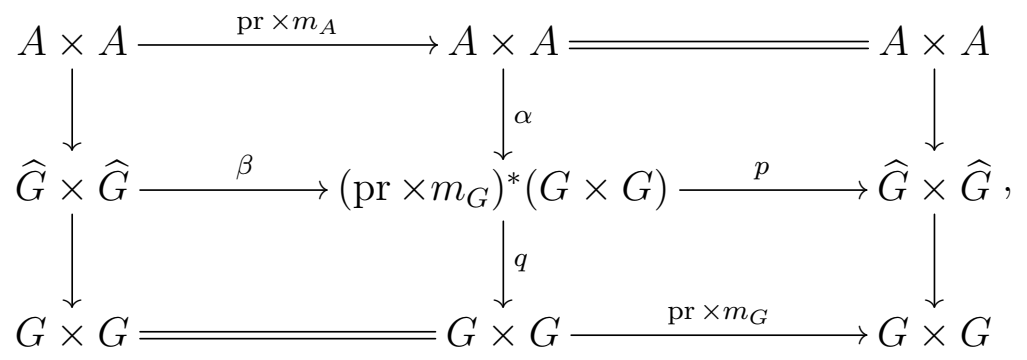

where $p$ and $q$ are the canonical maps and $\alpha$ and $\beta$ are induced maps into the pull-back. Since $\mathrm{pr} \times m_{G}$ is a diffeomorphism and the pull-back is functorial it follows that $p$ is a diffeomorphism. Since pr $\times m_{A}$ is an isomorphism of Lie groups we also have that $\beta$ is invertible.

The remainder of this section will be devoted to the proof of the following Theorem.

Theorem 2.17. Given a morphism $A \stackrel{\mu}{\rightarrow} B$ of abelian Lie groups and an arbitrary Lie group $G$ we have a well-defined map

$$
\check{H}^{3}\left(\mathbf{B} G_{\bullet}, A \stackrel{\mu}{\rightarrow} B\right) \rightarrow \operatorname{Ext}(\underline{G},[A \stackrel{\mu}{\rightarrow} B]),
$$

where $\operatorname{Ext}(\underline{G},[A \stackrel{\mu}{\rightarrow} B])$ denotes the equivalence classes of central extensions of $\underline{G}$ by $[A \stackrel{\mu}{\rightarrow} B]$.

The proof of this theorem will be finished in the end of Section 2.3. We first warm up with the following construction which also gives a geometric interpretation of the Čech cohomology of a constant simplicial manifold. Note that we will throughout assume that the occurring Čech cocycles are normalized, which is justified by Corollary 2.6.

\subsubsection{The principal bundle structure}

For a morphism $A \stackrel{\mu}{\rightarrow} B$ of abelian Lie groups and a manifold $M$, viewed as a constant simplicial manifold, let $(\eta, \gamma)$ be a 2-cocycle representing an element in the hypercohomology $\check{H}^{2}\left(M, A \stackrel{\mu}{\rightarrow} B\right.$ ) (cf. Remark 2.7), that is, there is an open cover $\left(U_{i}\right)_{i \in I}$ on $M$ with smooth maps

$$
\eta: U_{[2]} \rightarrow A, \quad \gamma: U_{[1]} \rightarrow B,
$$

satisfying $D_{3}(\eta, \gamma)=0$, i.e., $\mu \circ \eta=\check{\delta}(\gamma)$ and $\check{\delta}(\eta)=0$. Here, to simplify the notation, we refer for $q \geq 0$ to $U_{[q]}$ as the disjoint union

$$
U_{[q]}:=\coprod_{i_{0}, \ldots, i_{q} \in I} U_{i_{0}} \cap \cdots \cap U_{i_{q}}
$$


of $(q+1)$-fold intersections. The cocycle $(\gamma, \eta)$ defines a principal 2-bundle $\mathcal{P}_{(\gamma, \eta)} \rightarrow M$ (cf. [NW11, Woc11b]). It is presented by a Lie groupoid denoted by $U_{[1]} \times_{(\eta, \gamma)} B \times A \rightrightarrows$ $U_{[0]} \times B$. The structure maps are given by

$\mathbf{t}\left(x_{i j}, b, a\right)=\left(x_{i}, b\right), \quad \mathbf{s}\left(x_{i j}, b, a\right)=\left(x_{j}, b+\mu(a)+\gamma_{i j}(x)\right), \quad \forall\left(x_{i j}, b, a\right) \in U_{i j} \times B \times A$,

and

$$
\begin{gathered}
\left(x_{i j}, b, a\right) \cdot\left(x_{j k}, b^{\prime}, a^{\prime}\right)=\left(x_{i k}, b, a+a^{\prime}-\eta_{i j k}(x)\right), \\
e\left(x_{i}, b\right)=\left(x_{i i}, b, 0\right), \\
\left(x_{i j}, b, a\right)^{-1}=\left(x_{j i}, b+\mu(a)+\gamma_{i j}(x),-a\right) .
\end{gathered}
$$

Then $D_{3}(\eta, \gamma)=0$ implies that (2.22) is compatible with the source and target maps and is associative.

For the description of bundle morphisms in local coordinates it is often necessary to change the open covers describing a principal 2-bundle. Unlike in the case of principal bundles, different choice of $\eta, \gamma$ representing the same class in $H^{2}(M, A \stackrel{\mu}{\rightarrow} B)$ do not give isomorphic principal 2-bundles, but only essentially equivalent groupoids with isomorphic principal bundle structure:

Theorem 2.18. ([Woc11b, Theorem 2.22]) Given a strict 2-group $A \stackrel{\mu}{\rightarrow} B$, the equivalence classes of principal $[A \rightarrow B]$-2-bundles $\mathcal{P} \rightarrow M$ are classified by $\check{H}^{2}(M, A \stackrel{\mu}{\rightarrow} B)$.

\subsubsection{The Lie 2-group structure}

Now starting from a cocycle $\phi \in \check{Z}_{U}^{3}(\mathbf{B} G \bullet, A \stackrel{\mu}{\rightarrow} B)$ of a given simplicial covering $\left(U_{i}^{(n)}\right)_{i \in I^{(n)}}$, we set off to construct the groupoid which provides the base space for $\widehat{G}$ in the central extension $[A \stackrel{\mu}{\rightarrow} B] \rightarrow \widehat{G} \rightarrow \underline{G}$.

First of all, we need to construct a $A \stackrel{\mu}{\rightarrow} B$ principal 2-bundle over $G$. There is a homomorphism

$\check{Z}_{\mathcal{U}}^{3}\left(\mathbf{B} G_{\bullet}, A \stackrel{\mu}{\rightarrow} B\right) \stackrel{\tau}{\rightarrow} \check{Z}_{\mathcal{U}}^{2}(G, A \stackrel{\mu}{\rightarrow} B), \quad \tau\left(\phi^{1,1,0}, \phi^{1,2,-1}, \phi^{2,1,-1}, \phi^{2,0,0}, \phi^{3,0,-1}\right)=\left(\phi^{1,1,0}, \phi^{1,2,-1}\right)$

inducing on the level of cohomology classes the edge homomorphism

$$
\check{H}^{3}\left(\mathbf{B} G_{\bullet}, A \stackrel{\mu}{\rightarrow} B\right) \rightarrow \check{H}^{2}(G, A \stackrel{\mu}{\rightarrow} B)
$$

(note that $\check{H}_{\mathcal{U}}^{2}(G, A \stackrel{\mu}{\rightarrow} B)$ is by definition the cohomology of the double complex $\check{C}^{1, q, r}$ for $p=1$ constant from (2.7)).

The 2-cocycle $\left(\phi^{1,1,0}, \phi^{1,2,-1}\right)$ gives us the desired principal 2-bundle via the construction in Section 2.2.1. We call this Lie groupoid $\Gamma[\phi]$ and it will serve as the underlying 
Lie groupoid of our Lie 2-group. Notice that $\left(U_{i}^{(1)}\right)_{i \in I^{(1)}}$ is a covering of $G$, thus by the above discussion we have

$$
\Gamma[\phi]_{0}=U_{[0]}^{(1)} \times B, \quad \Gamma[\phi]_{1}=U_{[1]}^{(1)} \times B \times A .
$$

Here the lower indices denote disjoint union of intersections (see (2.21)). For this section we will switch back to the notation for cocycles that we used in Section 2.1. Then in this notation the groupoid multiplication on $\Gamma[\phi]$ is given by

$$
\left(u_{0}, u_{1}, b, a\right) \cdot\left(u_{1}, u_{2}, b^{\prime}, a^{\prime}\right)=\left(u_{0}, u_{2}, b, a+a^{\prime}-\phi^{1,2,-1}\left(u_{0}, u_{1}, u_{2}\right)\right)
$$

where we have also identified the intersection $U_{i_{0}} \cap U_{i_{1}}$ with the pull-back $U_{i_{0}} \times_{G} U_{i_{1}}$.

We recall that $\mathrm{B} G$, the nerve of $(G \rightrightarrows *)$, is a simplicial manifold as established in Example 2.1. We use $d_{2}, d_{0}: \mathbf{B} G_{2}=G \times G \rightarrow \mathbf{B} G_{1}=G$ to pullback the 2-cocycle $\left(\phi^{(1,1,0)}, \phi^{(1,2,-1)}\right)$ on $G$. Then we obtain two such 2 -cocycles $d_{2}^{*}\left(\phi^{(1,1,0)}, \phi^{(1,2,-1)}\right)$ and $d_{0}^{*}\left(\phi^{(1,1,0)}, \phi^{(1,2,-1)}\right)$ on $G \times G$. The above construction gives us a $(A \stackrel{\mu}{\rightarrow} B)^{\times 2}$ principal 2-bundle on $G \times G$, whose underlining Lie groupoid, denoted by $\Gamma^{2}[\phi]$, is given by

$$
U_{[1]}^{(2)} \times B^{\times 2} \times A^{\times 2} \rightrightarrows U_{[0]}^{(2)} \times B^{\times 2}
$$

Moreover, there is a Lie groupoid morphism $\left(d_{0}, d_{2}\right): \Gamma^{2}[\phi] \rightarrow \Gamma[\phi] \times \Gamma[\phi]$ defined on $U_{[1]}^{(2)} \times B^{\times 2} \times A^{\times 2} \rightarrow U_{[1]}^{(1)} \times B \times A \times U_{[1]}^{(1)} \times B \times A$ by

$$
\left(v_{0}, v_{1}, b_{0}, b_{1}, a_{0}, a_{1}\right) \mapsto\left(d_{0}\left(v_{0}\right), d_{0}\left(v_{1}\right), b_{0}, a_{0}\right) \times\left(d_{2}\left(v_{0}\right), d_{2}\left(v_{1}\right), b_{1}, a_{1}\right) .
$$

We will throughout define a Lie groupoid morphism on the space of arrows since this determines the morphism on the objects uniquely. Notice that $d_{0}^{*} \Gamma[\phi] \times d_{2}^{*} \Gamma[\phi]$ is given by

$$
{U^{\prime}}_{[1]}^{(2)} \times A^{\times 2} \times B^{\times 2} \rightrightarrows{U^{\prime}}_{[0]}^{(2)} \times B^{\times 2},
$$

where $\left(U_{i_{0}, i_{1}}^{\prime(2)}:=U_{i_{0}} \times U_{i_{1}}\right)_{\left(i_{0}, i_{1}\right) \in I^{(1)} \times I^{(1)}}$ is the product open covering of $G \times G$. Note that this is exactly the same as the coarsest common refinement of the pull-back covers $d_{0}^{*}\left(U_{i}^{(1)}\right)_{i \in I^{(1)}}$ and $d_{2}^{*}\left(U_{i}^{(1)}\right)_{i \in I^{(1)}}$, thus it contains $U^{(2)}$ as a subcovering. By [Woc11b, Corollary 2.23], the groupoid morphism (2.24) as the composition of the inclusion $\Gamma^{2}[\phi] \rightarrow d_{0}^{*} \Gamma[\phi] \times$ $d_{2}^{*} \Gamma[\phi]$ and the isomorphism $d_{0}^{*} \Gamma[\phi] \times d_{2}^{*} \Gamma[\phi] \cong \Gamma[\phi]^{\times 2}$ is a weak equivalence. Thus $\Gamma^{2}[\phi]$ and $\Gamma[\phi]^{\times 2}$ are Morita equivalent. Similarly, we use $d_{0} d_{0}, d_{2} d_{0}, d_{2} d_{2}: \mathbf{B} G_{3} \rightarrow \mathbf{B} G_{1}$ to pullback the 2 -cocycle $\left(\phi^{(1,1,0)}, \phi^{(1,2,-1)}\right)$. This gives us a Lie groupoid $\Gamma^{3}[\phi]$ which is a $(A \stackrel{\mu}{\rightarrow} B)^{\times 3}$ principal 2-bundle on $G \times G \times G$. Moreover the map $\left(d_{2} d_{2}, d_{2} d_{0}, d_{0} d_{0}\right)$ gives arise to a Morita equivalence between $\Gamma^{3}[\phi]$ the product of three copies of $\Gamma[\phi]$.

We now define the multiplication morphism $m: \Gamma^{2}[\phi] \rightarrow \Gamma[\phi]$ by

$$
\left(v_{0}, v_{1}, b_{0}, b_{1}, a_{0}, a_{1}\right) \mapsto\left(d_{1}\left(v_{0}\right), d_{1}\left(v_{1}\right), b_{0}+b_{1}+\phi^{2,0,0}\left(v_{0}\right), a_{0}+a_{1}+\phi^{2,1,-1}\left(v_{0}, v_{1}\right)\right)
$$


Since the morphism $\Gamma^{2}[\phi] \rightarrow \Gamma[\phi] \times \Gamma[\phi]$ is a weak equivalence, the span

$$
\Gamma[\phi] \times \Gamma[\phi] \leftarrow \Gamma^{2}[\phi] \stackrel{m}{\rightarrow} \Gamma[\phi]
$$

defines a generalized morphism $\tilde{m}: \Gamma[\phi] \times \Gamma[\phi] \rightarrow \Gamma[\phi]$. That (2.25) is indeed a Lie groupoid morphism follows from (2.13) and (2.9) as follows. Keep in mind that in $\Gamma^{2}[\phi]$ we have

$$
\begin{aligned}
& \mathbf{t}\left(v_{0}, v_{1}, b_{0}, b_{1}, a_{0}, a_{1}\right)=\left(v_{0}, b_{0}, b_{1}\right), \\
& \mathbf{s}\left(v_{0}, v_{1}, b_{0}, b_{1}, a_{0}, a_{1}\right)=\left(v_{1}, b_{0}+\mu\left(a_{0}\right)+\phi^{1,1,0}\left(d_{0}\left(v_{0}\right), d_{0}\left(v_{1}\right)\right), b_{1}+\mu\left(a_{1}\right)+\phi^{1,1,0}\left(d_{2}\left(v_{0}\right), d_{2}\left(v_{1}\right)\right)\right), \\
& \left(v_{0}, v_{1}, b_{0}, b_{1}, a_{0}, a_{1}\right) \cdot\left(v_{1}, v_{2}, b_{0}^{\prime}, b_{1}^{\prime}, a_{0}^{\prime}, a_{1}^{\prime}\right) \\
& =\left(v_{0}, v_{2}, b_{0}, b_{1}, a_{0}+a_{0}^{\prime}-\phi^{1,2,-1}\left(d_{2}\left(v_{0}\right), d_{2}\left(v_{1}\right), d_{2}\left(v_{2}\right)\right), a_{1}+a_{1}^{\prime}-\phi^{1,2,-1}\left(d_{0}\left(v_{0}\right), d_{0}\left(v_{1}\right), d_{0}\left(v_{2}\right)\right)\right) .
\end{aligned}
$$

Then $m \circ \mathbf{t}=$ to $m$ holds by definition and we have

$$
\begin{aligned}
& m \circ \mathbf{s}\left(v_{0}, v_{1}, b_{0}, b_{1}, a_{0}, a_{1}\right) \\
= & \left(d_{1}\left(v_{1}\right), b_{0}+\mu\left(a_{0}\right)+\phi^{1,1,0}\left(d_{2}\left(v_{0}\right), d_{2}\left(v_{1}\right)\right)+b_{1}+\mu\left(a_{1}\right)+\phi^{1,1,0}\left(d_{0}\left(v_{0}\right), d_{0}\left(v_{1}\right)\right)+\phi^{2,0,0}\left(v_{1}\right)\right)
\end{aligned}
$$$$
\operatorname{som}\left(v_{0}, v_{1}, b_{0}, b_{1}, a_{0}, a_{1}\right)=\left(d_{1}\left(v_{1}\right), b_{0}+b_{1}+\phi^{2,0,0}\left(v_{0}\right)+\mu\left(a_{0}+a_{1}\right)+d_{0,0,1}\left(\phi^{2,1,-1}\left(v_{0}, v_{1}\right)\right)+\phi^{1,1,0}\left(d_{1}\left(v_{0}\right), d_{1}\left(v_{1}\right)\right)\right) \text {. }
$$

Thus $m \circ \mathbf{s}=\mathbf{s} \circ m$ is equivalent to (2.13). Similarly, we have on one hand

$$
\begin{aligned}
& m\left(\left(v_{0}, v_{1}, b_{0}, b_{1}, a_{0}, a_{1}\right) \cdot\left(v_{1}, v_{2}, b_{0}^{\prime}, b_{1}^{\prime}, a_{0}^{\prime}, a_{1}^{\prime}\right)\right) \\
= & m\left(v_{0}, v_{2}, b_{0}, b_{1}, a_{0}+a_{0}^{\prime}-\phi^{1,2,-1}\left(d_{2}\left(v_{0}\right), d_{2}\left(v_{1}\right), d_{2}\left(v_{2}\right)\right), a_{1}+a_{1}^{\prime}-\phi^{1,2,-1}\left(d_{0}\left(v_{0}\right), d_{0}\left(v_{1}\right), d_{0}\left(v_{2}\right)\right)\right) \\
= & \left(d_{1}\left(v_{0}\right), d_{1}\left(v_{2}\right), b_{0}+b_{1}+\phi^{2,0,0}\left(v_{0}\right),\right. \\
& \left.a_{0}+a_{0}^{\prime}-\phi^{1,2,-1}\left(d_{2}\left(v_{0}\right), d_{2}\left(v_{1}\right), d_{2}\left(v_{2}\right)\right)+a_{1}+a_{1}^{\prime}-\phi^{1,2,-1}\left(d_{0}\left(v_{0}\right), d_{0}\left(v_{1}\right), d_{0}\left(v_{2}\right)\right)+\phi^{2,1,-1}\left(v_{0}, v_{2}\right)\right),
\end{aligned}
$$

and on the other hand

$$
\begin{aligned}
& m\left(v_{0}, v_{1}, b_{0}, b_{1}, a_{0}, a_{1}\right) \cdot m\left(v_{1}, v_{2}, b_{0}^{\prime}, b_{1}^{\prime}, a_{0}^{\prime}, a_{1}^{\prime}\right) \\
= & \left(d_{1}\left(v_{0}\right), d_{1}\left(v_{1}\right), b_{0}+b_{1}+\phi^{2,0,0}\left(v_{0}\right),\right. \\
& \left.a_{0}+a_{1}+\phi^{2,1,-1}\left(v_{0}, v_{1}\right)\right) \cdot\left(d_{1}\left(v_{1}\right), d_{1}\left(v_{2}\right), b_{0}^{\prime}+b_{1}^{\prime}+\phi^{2,0,0}\left(v_{1}\right), a_{0}^{\prime}+a_{1}^{\prime}+\phi^{2,1,-1}\left(v_{1}, v_{2}\right)\right) \\
= & \left(d_{1}\left(v_{0}\right), d_{1}\left(v_{2}\right), b_{0}+b_{1}+\phi^{2,0,0}\left(v_{0}\right),\right. \\
& \left.a_{0}+a_{1}+\phi^{2,1,-1}\left(v_{0}, v_{1}\right)+a_{0}^{\prime}+a_{1}^{\prime}+\phi^{2,1,-1}\left(v_{1}, v_{2}\right)-\phi^{1,2,-1}\left(d_{1}\left(v_{0}\right), d_{1}\left(v_{1}\right), d_{1}\left(v_{2}\right)\right)\right) .
\end{aligned}
$$

Thus $m$ and · commute iff (2.9) holds.

Like the multiplication morphism, the associator will not be defined on $\Gamma[\phi]^{\times 3}$, but on the Morita equivalent Lie groupoid $\Gamma^{3}[\phi]$. There are two Lie groupoid morphisms $f_{1}, f_{2}: \Gamma^{3}[\phi] \rightarrow \Gamma[\phi]$ defined on $U_{[1]}^{(3)} \times B^{\times 3} \times A^{\times 3} \rightarrow\left(U_{[1]}^{(1)} \times B \times A\right)^{\times 3}$ by

$f_{1}:\left(w_{0}, w_{1}, b_{0}, b_{1}, b_{2}, a_{0}, a_{1}, a_{2}\right) \mapsto\left(d_{1}\left(d_{2}\left(w_{0}\right)\right), d_{1}\left(d_{2}\left(w_{1}\right)\right), b_{0}+b_{1}+b_{2}+d_{2}{ }^{*} \phi^{2,0,0}\left(w_{0}\right)+d_{0}{ }^{*} \phi^{2,0,0}\left(w_{0}\right)\right.$,

$$
\left.a_{0}+a_{1}+a_{2}+d_{2}{ }^{*} \phi^{2,1,-1}\left(w_{0}, w_{1}\right)+d_{0}{ }^{*} \phi^{2,1,-1}\left(w_{0}, w_{1}\right)\right)
$$

$f_{2}:\left(w_{0}, w_{1}, b_{0}, b_{1}, b_{2}, a_{0}, a_{1}, a_{2}\right) \mapsto\left(d_{1}\left(d_{1}\left(w_{0}\right)\right), d_{1}\left(d_{1}\left(w_{1}\right)\right), b_{0}+b_{1}+b_{2}+d_{1}{ }^{*} \phi^{2,0,0}\left(w_{0}\right)+d_{3}{ }^{*} \phi^{2,0,0}\left(w_{0}\right)\right.$,

$$
\left.a_{0}+a_{1}+a_{2}+d_{1}{ }^{*} \phi^{2,1,-1}\left(w_{0}, w_{1}\right)+d_{3}{ }^{*} \phi^{2,1,-1}\left(w_{0}, w_{1}\right)\right) .
$$


As before, $f_{1}$ and $f_{2}$ are Lie groupoid morphisms by (2.13) and (2.9) and since $d_{1} \circ d_{1}=$ $d_{1} \circ d_{2}$. Note that $f_{1}$ is the same as $m \circ(\mathrm{id} \times m)$, restricted from $(\mathrm{id} \times m)^{*} \Gamma^{2}[\phi]$ to $\Gamma^{3}[\phi]$ and that $f_{2}$ is the same as $m \circ(m \times \mathrm{id})$, restricted from $(m \times \mathrm{id})^{*} \Gamma^{2}[\phi]$ to $\Gamma^{3}[\phi]$.

There is a smooth natural transformation $\alpha: f_{1} \Leftarrow f_{2}$, which is a map $\alpha: \Gamma^{3}[\phi]_{0} \rightarrow \Gamma[\phi]_{1}=$ $U_{[1]}^{(2)} \times B \times A$ defined by

$\left(w_{0}, b_{0}, b_{1}, b_{2}\right) \mapsto\left(d_{1} d_{2}\left(w_{0}\right), d_{1} d_{2}\left(w_{0}\right), b_{0}+b_{1}+b_{2}+d_{2}{ }^{*} \phi^{2,0,0}\left(w_{0}\right)+d_{0}{ }^{*} \phi^{2,0,0}\left(w_{0}\right), \phi^{3,0,-1}\left(w_{0}\right)\right)$

To verify this, we only need to show that

$$
f_{1}(\gamma) \cdot \alpha(\mathbf{s}(\gamma))=\alpha(\mathbf{t}(\gamma)) \cdot f_{2}(\gamma)
$$

for $\gamma \in \Gamma^{3}[\phi]_{1}$ (source-target matching is equivalent to (2.13) and (2.14) and $d_{1} d_{1}=$ $\left.d_{1} d_{2}\right)$. Take $\gamma=\left(w_{0}, w_{1}, b_{0}, b_{1}, b_{2}, a_{0}, a_{1}, a_{2}\right)$, then

$$
\begin{aligned}
\mathbf{s}(\gamma)= & \left(w_{1}, b_{0}+\mu\left(a_{0}\right)+\phi^{1,1,0}\left(d_{0}\left(d_{0}\left(w_{0}\right)\right), d_{0}\left(d_{0}\left(w_{1}\right)\right)\right), b_{1}+\mu\left(a_{1}\right)+\phi^{1,1,0}\left(d_{2}\left(d_{0}\left(w_{0}\right)\right), d_{2}\left(d_{0}\left(w_{1}\right)\right),\right.\right. \\
& \left.b_{2}+\mu\left(a_{2}\right)+\phi^{1,1,0}\left(d_{2}\left(d_{2}\left(w_{0}\right)\right), d_{2}\left(d_{2}\left(w_{1}\right)\right)\right)\right)
\end{aligned}
$$

and $\mathbf{t}(\gamma)=\left(w_{0}, b_{0}, b_{1}, b_{2}\right)$. Then

$$
\begin{aligned}
f_{1}(\gamma) \cdot \alpha(\mathbf{s}(\gamma))= & \left(d_{1} d_{2}\left(w_{0}\right), d_{1} d_{2}\left(w_{1}\right), b_{0}+b_{1}+b_{2}+d_{2}{ }^{*} \phi^{2,0,0}\left(w_{0}\right)+d_{0}{ }^{*} \phi^{2,0,0}\left(w_{0}\right),\right. \\
& \left.a_{0}+a_{1}+a_{2}+d_{2}{ }^{*} \phi^{2,1,-1}\left(w_{0}, w_{1}\right)+d_{0}{ }^{*} \phi^{2,1,-1}\left(w_{0}, w_{1}\right)+\phi^{3,0,-1}\left(w_{1}\right)\right),
\end{aligned}
$$

and

$$
\begin{aligned}
\alpha(\mathbf{t}(\gamma)) \cdot f_{2}(\gamma) & =\left(d_{1}\left(d_{2}\left(w_{0}\right)\right), d_{1}\left(d_{1}\left(w_{1}\right)\right), b_{0}+b_{1}+b_{2}+d_{2}{ }^{*} \phi^{2,0,0}\left(w_{0}\right)+d_{0}{ }^{*} \phi^{2,0,0}\left(w_{0}\right), \phi^{3,0,-1}\left(w_{0}\right)\right. \\
& \left.+a_{0}+a_{1}+a_{2}+d_{1}{ }^{*} \phi^{2,1,-1}\left(w_{0}, w_{1}\right)+d_{3}{ }^{*} \phi^{2,1,-1}\left(w_{0}, w_{1}\right)\right)
\end{aligned}
$$

Thus (2.27) is equivalent to (2.10). In the end, the same argument as before shows that

$$
d_{1,0,0} \phi^{3,0,-1}=0
$$

is equivalent to the coherence condition that the associator $\alpha$ has to satisfy (see also [SP11, Theorem 99]).

For the unit we choose some $i \in I^{(1)}$ such that the identity $e$ of $G$ is in $U_{i}^{(1)}$. Then the unit of $\widehat{G}$ is given by the groupoid morphism, uniquely determined by

$$
u: * \rightarrow \Gamma[\phi], \quad * \mapsto(e, 0) \in U_{i} \times B .
$$

Then the composition $m \circ(\mathrm{id} \times u)$ is defined as a smooth functor on the Lie groupoid $s_{1}^{*} \Gamma^{2}[\phi]$, where

$$
s_{1}: G \times * \cong G \rightarrow G \times G
$$

is the embedding into the first factor. Since $\left(s_{1}^{-1}\left(U_{i}^{(2)}\right)\right)_{i \in I^{(2)}}$ is a refinement of $\left(d_{2}\left(U_{i}^{(2)}\right)\right)_{i \in I^{(2)}}$ and since $\left(d_{2}\left(U_{i}^{(2)}\right)\right)_{i \in I^{(2)}}$ is a refinement of $\left(U_{i}^{(1)}\right)_{i \in I^{(1)}}$ by assumption, the natural inclusion $s_{1}^{*} \Gamma^{2}[\phi] \hookrightarrow \Gamma[\phi]$ is a weak equivalence. It thus suffices to check that

$$
s_{1}^{*} \Gamma^{2}[\phi] \rightarrow \Gamma^{2}[\phi] \stackrel{m}{\rightarrow} \Gamma[\phi]
$$


is also equal to the inclusion $s_{1}^{*} \Gamma^{2}[\phi] \hookrightarrow \Gamma[\phi]$. An arbitrary morphism in $s_{1}^{*} \Gamma^{2}[\phi]$ now is of the form $\left(v_{0}, v_{1}, b_{0}, 0, a_{0}, 0\right)$, where $v_{0}=s_{1}\left(u_{0}\right)$ and $v_{1}=s_{1}\left(u_{1}\right)$. Thus the simplicial identities and our normalization conditions imply that

$m\left(v_{0}, v_{1}, b_{0}, 0, a_{0}, 0\right)=\left(d_{1}\left(v_{0}\right), d_{1}\left(v_{1}\right), b_{0}+\phi^{2,0,1}\left(v_{0}\right), a_{0}+\phi^{2,1,0}\left(v_{0}, v_{1}\right)\right)=\left(v_{0}, v_{1}, b_{0}, a_{0}\right)$.

This shows that the 2-morphism $m \circ(\mathrm{id} \times u) \Rightarrow$ id can actually be taken to be represented by the identity natural transformation on the inclusion $s_{1}^{*} \Gamma^{2}[\phi] \hookrightarrow \Gamma[\phi]$. The same argument shows that $u$ is also a strict right unit. A categorification of the argument in Remark 2.16 now shows that (2.20) holds for $\Gamma[\phi]$. Thus $\Gamma[\phi]$, together with $m, u$ and $\alpha$ is indeed a Lie 2-group, denoted $\widehat{G}_{\phi}$.

Having fixed the choice of $i \in I^{(1)}$ with $e \in U_{i}$, there is a canonical morphism of Lie groupoids $(A \times B \rightrightarrows B) \rightarrow \Gamma[\phi]$, given by

$$
(a, b) \mapsto\left(e_{i}, e_{i}, a, b\right) \in U_{i} \cap U_{i} \times B \times A .
$$

(where we again have identified $U_{i} \times_{G} U_{i}$ with $U_{i} \cap U_{i} \subseteq G$ ) one easily checks that this defines a morphism of the associated Lie 2-groups $[A \stackrel{\mu}{\rightarrow} B] \stackrel{p}{\rightarrow} \widehat{G}_{\phi}$ with trivial $F_{2}$ and $F_{0}$. Likewise, the morphism $\Gamma[\phi] \rightarrow \underline{G}$, given by

$$
\left(u_{0}, u_{1}, b, a\right) \mapsto u_{0}
$$

gives a homomorphism of Lie 2-groups $\widehat{G}_{\phi} \stackrel{q}{\rightarrow} \underline{G}$. From this it is obvious that the composition $[A \stackrel{\mu}{\rightarrow} B] \rightarrow \widehat{G}_{\phi} \rightarrow \underline{G}$ is the trivial morphism $[A \stackrel{\mu}{\rightarrow} B] \rightarrow * \rightarrow \underline{G}$. We thus have an extension

$$
[A \stackrel{\mu}{\rightarrow} B] \rightarrow \widehat{G}_{\phi} \rightarrow \underline{G} .
$$

That this is in fact a central extension follows from the fact that we considered the action of $\underline{G}$ on $[A \stackrel{\mu}{\rightarrow} B]$ as trivial (cf. [SP11, Lemma 84]). We thus arrive at the following

Proposition 2.19. For $\phi \in \check{Z}_{U}^{3}\left(\mathbf{B} G_{\bullet}, A \stackrel{\mu}{\rightarrow} B\right)$, the Lie 2-group $\widehat{G}_{\phi}$, together with the morphisms $[A \stackrel{\mu}{\rightarrow} B] \stackrel{p}{\rightarrow} \widehat{G}_{\phi}, \widehat{G}_{\phi} \stackrel{q}{\rightarrow} \underline{G}$ is a central extension of Lie 2-groups.

\subsection{Cohomologous cocycles}

Now suppose that we have two cohomologous 3-cocycles $\phi$ and $\widetilde{\phi}$ satisfying Equations (2.18), (2.19), (2.15), (2.16) and (2.17) with a 2-cochain $\psi$. Since different covers lead to weak equivalences we may as well assume that $\phi$ and $\widetilde{\phi}$ live on the same cover $U$. In this case the 1-morphism $f: \widehat{G}_{\phi} \rightarrow \widehat{G}_{\widetilde{\phi}}$ is given by the smooth functor

$$
F: \Gamma[\phi] \rightarrow \Gamma[\widetilde{\phi}], \quad\left(u_{0}, u_{1}, b, a\right) \mapsto\left(u_{0}, u_{1}, b+\psi^{1,0,0}\left(u_{0}\right), a+\psi^{1,1,-1}\left(u_{0}, u_{1}\right)\right),
$$

the smooth natural transformation $F_{2}: m_{G_{\phi}} \circ(F \times F) \Rightarrow F \circ m_{G_{\tilde{\phi}}}$,

$$
\left(v_{0}, b_{0}, b_{1}\right) \mapsto\left(d_{1}\left(v_{0}\right), d_{1}\left(v_{0}\right), b_{0}+b_{1}+\phi^{2,0,0}\left(v_{0}\right)+\psi^{1,0,0}\left(d_{1}\left(v_{0}\right)\right), \psi^{2,0,-1}\left(v_{0}\right)\right)
$$


and the smooth natural transformation $F_{0}: F \circ u \Rightarrow u^{\prime}, * \mapsto\left(e_{i}, e_{\tilde{i}}, 0,0\right)$.

Indeed, (2.18) implies that $F$ preserves source and target, and (2.15) implies that $F$ preserves the groupoid multiplication. Thus $F$ is a groupoid morphism. Moreover, (2.19) and (2.16) imply source-target matching for $F_{2}$. That $F_{2}$ satisfies the coherence condition is then implied by (2.17). The equivalence between the central extensions is then completed by the smooth natural transformation

$$
\lambda: F \circ p \Rightarrow p^{\prime}, \quad(a, b) \mapsto\left(e_{i}, e_{\tilde{i}}, b, a\right)
$$

\section{Geometric cocycle constructions}

This section describes a geometric way for constructing differentiable cocycles on $G$ from Lie algebra cocycles on $\mathfrak{g}$ and is the heart of the paper.

\subsection{Locally smooth cocycles}

The continuous second Lie algebra cohomology $H_{c}^{2}(\mathfrak{g}, \mathfrak{z})$ classifies topologically split central extensions

$$
\mathfrak{z} \rightarrow \widehat{\mathfrak{g}} \rightarrow \mathfrak{g}
$$

of $\mathfrak{g}$ by $\mathfrak{z}$. Given a 2-cocycle $\omega$ representing a class $[\omega] \in H^{2}(\mathfrak{g}, \mathfrak{z})$, we first review the construction from [Woc11a] of a locally smooth cocycle $(\widetilde{F}, \widetilde{\Theta})$ integrating $\omega$.

Definition 3.1. Let $G$ be an arbitrary Lie group and $A$ an abelian Lie group. Then we set

$$
C_{\text {loc }}^{n}(G, A):=\left\{f: G^{n} \rightarrow A \mid f \text { is smooth on some identity neighborhood }\right\} .
$$

On $C_{\text {loc }}^{n}(G, A)$ we have the usual group differential

$$
\begin{aligned}
\mathrm{d}_{\mathrm{gp}}: C_{\mathrm{loc}}^{n}(G, A) \rightarrow C_{\mathrm{loc}}^{n+1}(G, A), \quad \mathrm{d}_{\mathrm{gp}} f\left(g_{0}, \ldots g_{n}\right)= \\
\quad g_{0} . f\left(g_{1}, \ldots, g_{n}\right)-\sum_{i=0}^{n-1}(-1)^{i} f\left(g_{0}, \ldots, g_{i} g_{i+1}, \ldots, g_{n}\right)-(-1)^{n} f\left(g_{0}, \ldots, g_{n-1}\right) .
\end{aligned}
$$

The corresponding cohomology groups will be denoted by $H_{\mathrm{loc}}^{n}(G, A)$. If $A \stackrel{\mu}{\rightarrow} B$ is a morphism of abelian Lie groups, then an $(A \stackrel{\mu}{\rightarrow} B)$-valued locally smooth group cocycle on $G$ (shortly called locally smooth cocycle if the setting is understood) consists of two maps $F \in C_{\mathrm{loc}}^{2}(G, B)$ and $\Theta \in C_{\mathrm{loc}}^{3}(G, A)$ such that

$$
\mathrm{d}_{\mathrm{gp}} F=\mu \circ \Theta \quad \text { and } \quad \mathrm{d}_{\mathrm{gp}} \Theta=0 .
$$


Now $\widetilde{\Theta}$ is constructed as follows. For each $g, h \in G$, let $\widetilde{\alpha}(g): \Delta^{(1)} \rightarrow G$ be smooth with $\widetilde{\alpha}(g)(0)=e$ and $\widetilde{\alpha}(g)(1)=g$ and $\widetilde{\beta}(g, h): \Delta^{(2)} \rightarrow G$ be smooth with

$$
g . \widetilde{\alpha}(h)-\widetilde{\alpha}(g h)+\widetilde{\alpha}(g)=\partial_{\operatorname{sing}} \widetilde{\beta}(g, h),
$$

where $\partial_{\text {sing }}$ denotes the differential of singular chains. These maps exist since we assume $G$ to be 1-connected. In addition, we may choose these maps so that

$$
G \ni g \mapsto \widetilde{\alpha}(g) \in C_{*}^{\infty}\left(\Delta^{(1)}, G\right) \quad \text { and } \quad G^{2} \ni(g, h) \mapsto \widetilde{\beta}(g, h) \in C_{*}^{\infty}\left(\Delta^{(2)}, G\right)
$$

are smooth on an identity neighborhood $U$. In fact, if $\varphi: P \rightarrow \widetilde{P} \subseteq \mathfrak{g}$ is a chart for $G$ with $\varphi(e)=0$ and $\widetilde{P}$ convex and if we set $\widetilde{g}:=\varphi(g)$ and $\widetilde{g} \star \widetilde{h}:=\widetilde{g h}$, then we set

$$
\begin{aligned}
\widetilde{\alpha}(g)(t) & :=\varphi^{-1}(t \cdot \widetilde{g}) \\
\widetilde{\beta}(g, h)(s, t) & :=\varphi^{-1}(t(\widetilde{g} \star s \widetilde{h})+s(\widetilde{g} \star(1-t) \widetilde{h})) .
\end{aligned}
$$

for $g, h \in U$ and $U \subseteq P$ open with $U^{2} \subseteq P$ and $\varphi(U)$ convex (cf. [Woc11a, Lemma 1.7]). Since the maps

$U \times[0,1] \ni(g, t) \mapsto \widetilde{\alpha}(g)(t) \in G \quad$ and $\quad U \times U \times \Delta^{(2)} \ni(g, h,(s, t)) \mapsto \widetilde{\beta}(g, h)(s, t) \in G$

are smooth, the maps $g \mapsto \widetilde{\alpha}(g)$ and $(g, h) \mapsto \widetilde{\beta}(g, h)$ are smooth on $U$ and $U \times U$ respectively [GN14]. In addition, we fix some $V \subseteq U$ open with $e \in V, V^{2} \subseteq U$ and $V=V^{-1}$.

Lemma 3.2. ([Wocl la, Lemmas 1.5,1.6 and 1.7]) For $g, h, k \in G$ we have that

$$
\widetilde{\Theta}(g, h, k):=g \cdot \widetilde{\beta}(h, k)-\widetilde{\beta}(g h, k)+\widetilde{\beta}(g, h k)-\widetilde{\beta}(g, h)
$$

is a closed singular 2-chain on $G$ and thus defines an element of $H_{2}(G) \cong \pi_{2}(G)$. Moreover, the map $(g, h, k) \mapsto[\widetilde{\Theta}(g, h, k)]$ is a $\left(\pi_{2}(G) \rightarrow 0\right)$-valued locally smooth group cocycle.

From the building blocks of $\widetilde{\Theta}$, we can also construct a $\left(\pi_{2}(G) \stackrel{\text { per }_{\omega}}{\longrightarrow} \mathfrak{z}\right)$-valued cocycle as follows. We set

$$
\widetilde{F}(g, h):=\int_{\widetilde{\beta}(g, h)} \omega^{l}=\operatorname{per}_{\omega}(\widetilde{\beta}(g, h)) .
$$

Since $(g, h) \mapsto \widetilde{\beta}(g, h)$ is smooth on $V$ and since integration along a fixed integrand defines a smooth map $C^{\infty}\left(\Delta^{(2)}, G\right) \rightarrow \mathfrak{z}$ (see A), it follows that $\widetilde{F}$ is smooth on $V \times V$. From the definition, it follows directly that

$$
\widetilde{F}(h, k)-\widetilde{F}(g h, k)+\widetilde{F}(g, h k)-\widetilde{F}(g, h)=\int_{g . \widetilde{\beta}(h, k)-\widetilde{\beta}(g h, k)+\widetilde{\beta}(g, h k)-\widetilde{\beta}(g, h)} \omega^{l}=\operatorname{per}_{\omega}(\widetilde{\Theta}(g, h, k))
$$


(where we used the fact that $\omega^{l}$ is left-invariant) and thus $(\widetilde{F}, \widetilde{\Theta})$ is a $\left(\pi_{2}(G) \stackrel{\text { per }_{\omega}}{\longrightarrow} \mathfrak{z}\right)$ valued locally smooth cocycle on $G$. It has the property that it integrates $\omega$ (in an appropriate sense, cf. [Woc11a, Theorem 1.11]) and it is universal with this property [Woc11a, Corollary 1.18].

Eventually, we now come to the point how the cocycle $(\widetilde{F}, \widetilde{\Theta})$ is related to the integration procedure from [Nee02].

Proposition 3.3. If $\mathrm{im}\left(\operatorname{per}_{\omega}\right) \subseteq \mathfrak{z}$ is discrete and $q: \mathfrak{z} \rightarrow Z:=\mathfrak{z} / \operatorname{im}\left(\operatorname{per}_{\omega}\right)$ is the quotient map, then $f:=q \circ \widetilde{F}$ defines a locally smooth $(0 \rightarrow Z)$-valued cocycle. It integrates $\omega$ in the sense that $D([f])=[\omega]$, where

$$
D: H_{\mathrm{loc}}^{2}\left(G, \mathfrak{z} / \operatorname{per}_{\omega}\left(\pi_{2}(G)\right)\right) \rightarrow H_{c}^{2}(\mathfrak{g}, \mathfrak{z})
$$

is the differentiation homomorphism from [Nee02, Section 4].

Proof. Equation (3.5) shows that $\left(\mathrm{d}_{\mathrm{gp}} f\right)(g, h, k)$ vanishes in $Z$ and since $\widetilde{F}$ is smooth in a neighborhood of $(e, e)$, the same is true for $f=q \circ \widetilde{F}$. Since $f$ coincides with the cocycle constructed in [Nee02, Section 6] it integrates $\omega$ by [Nee02, Corollary 6.3].

Since (equivalence classes of) $(0 \rightarrow Z)$-valued locally smooth group cocycles are the same thing as central extensions of $G$ by $Z$ [Nee02], the previous proposition answers the integrability question for $\omega$ in the case of discrete $\operatorname{im}\left(\operatorname{per}_{\omega}\right)$.

\subsection{Differentiable cocycles}

The locally smooth cocycle from the previous section lacked the global smoothness properties of the group structure. In the case of ordinary groups, the locally smooth group cocycles induced a smooth structure on the whole group extension, turning it into an extension of Lie group. This procedure made heavy used of the associativity of the group multiplication and thus does not seem to work for higher groups any more.

In this section, we shall now enhance the construction from the previous section in an ad-hoc manner to a globally smooth object associated to the Lie algebra cocycle $\omega$, namely a differentiable cocycle with respect to an equivariant cover of $G$.

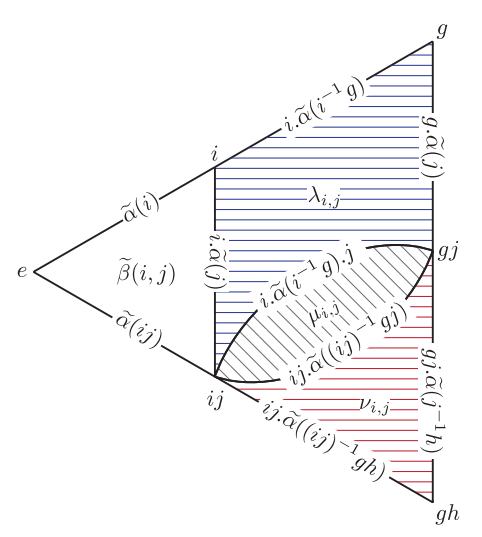

Figure 1: Construction of $\alpha_{i, j}$ (the hatched areas depend smoothly on $(g, h)$ and the blank areas are constant) 
We will now describe how to obtain the Čech cocycle describing the underlying 2-bundle from $(\widetilde{F}, \widetilde{\Theta})($ cf. [Woc11a, Remark 7.2]) The cocycle $(\widetilde{F}, \widetilde{\Theta})$ has the property that $\widetilde{F}$ is smooth on $U \times U$ and $\widetilde{\Theta}$ is smooth on $U \times U \times U$ for some identity neighborhood $U \subseteq G$. Let $V \subseteq U$ be open such that $e \in V, V=V^{-1}$ and $V^{2} \subseteq U$. From $V$ we obtain the open cover $\left(V_{i}\right)_{i \in G}$ when setting

$$
V_{i}:=i \cdot V
$$

We associate to $(\widetilde{F}, \widetilde{\Theta})$ (see (3.1), (3.4) and (3.3)) the cocycle

$$
\gamma_{i, j}: V_{i} \cap V_{j} \rightarrow \mathfrak{z}, \quad g \mapsto-\widetilde{F}\left(j, j^{-1} g\right)+\widetilde{F}\left(i, i^{-1} g\right)-\operatorname{per}_{\omega}\left(\widetilde{\Theta}\left(i, i^{-1} j, j^{-1} g\right)\right)
$$

$\eta_{i, j, l}: V_{i} \cap V_{j} \cap V_{l} \rightarrow \pi_{2}(G), \quad g \mapsto-\widetilde{\Theta}\left(j, j^{-1} l, l^{-1} g\right)+\widetilde{\Theta}\left(i, i^{-1} l, l^{-1} g\right)-\widetilde{\Theta}\left(i, i^{-1} j, j^{-1} g\right)$,

see Section 3.4 for an interpretation of this assignment.

From this definition, one immediately checks that $\gamma_{i, j}$ and $\eta_{i, j, l}$ satisfy (2.8) and (2.12) (recall that $\widetilde{F}$ and $\widetilde{\Theta}$ vanish whenever one of its arguments is $e$ ). That $\gamma_{i, j}$ depends smoothly on $g$ follows from

$$
\widetilde{F}\left(i, i^{-1} g\right)-\widetilde{F}\left(j, j^{-1} g\right)-\operatorname{per}_{\omega}\left(\widetilde{\Theta}\left(i, i^{-1} j, j^{-1} g\right)\right)=\widetilde{F}\left(i, i^{-1} j\right)-i \cdot \widetilde{F}\left(i^{-1} j, j^{-1} g\right)
$$

for $j^{-1} g \in V \subseteq U$ if $g$ in $V_{j}$ and if $V_{i} \cap V_{j} \neq \emptyset$, then $i^{-1} j \in V^{2} \subseteq U$. Similarly, one sees that $\eta_{i, j, l}$ depends smoothly on $g$ from

$$
\widetilde{\Theta}\left(i, i^{-1} j, j^{-1} g\right)-\widetilde{\Theta}\left(i, i^{-1} l, l^{-1} g\right)+\widetilde{\Theta}\left(j, j^{-1} l, l^{-1} g\right)=\widetilde{\Theta}\left(i, i^{-1} j, j^{-1} l\right)+i \cdot \widetilde{\Theta}\left(i^{-1} j, j^{-1} l, l^{-1} g\right)
$$

The construction from Sec-

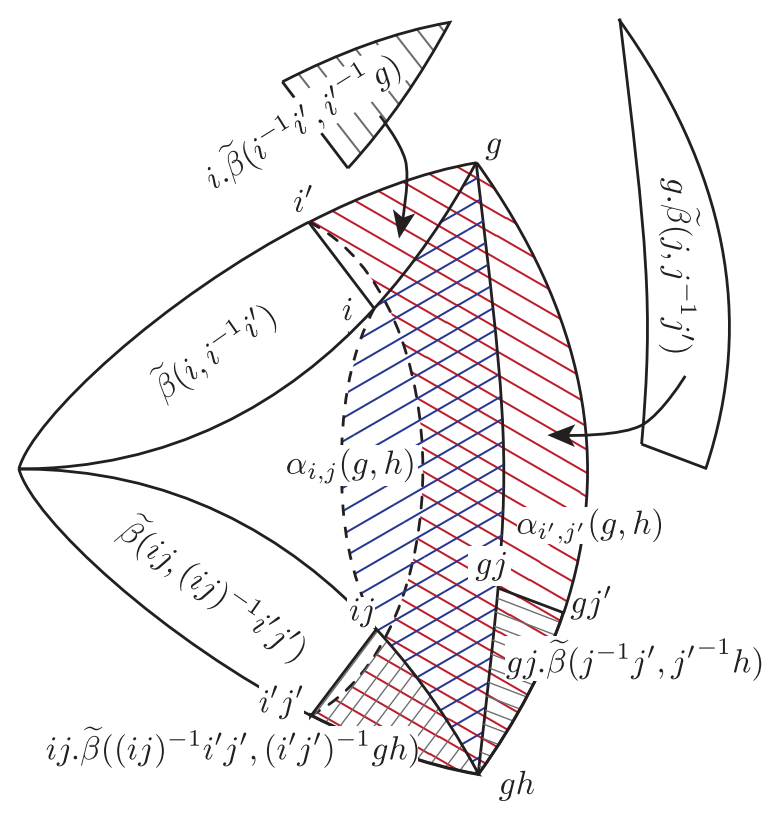

Figure 2: Construction of $\Phi_{(i, j),\left(i^{\prime}, j^{\prime}\right)}$ tion 2.2.1 now gives a Lie $\operatorname{groupoid} c=\left(V_{i}, \gamma_{i, j}, \eta_{i, j, l}\right)_{i, j, k \in G}$. We call this Lie groupoid from now on $\mathcal{G}_{\omega}$. We will justify this notation later on when showing that $\mathcal{G}_{\omega}$ does up to equivalence not depend on all the choices that we made).

This Lie groupoid will be shown to carry the structure of a Lie-2 group that integrates $\omega$ in the general case, regardless of the question whether $\operatorname{per}_{\omega}\left(\pi_{2}(G)\right)$ is discrete or not. 
In what follows, we stick to the notation introduced in Section 3.1. What we choose in addition is for each $j \in G$ some open identity neighborhood $W_{j} \subseteq V$ with the property that $j^{-1} W_{j} j \subseteq V$ and that $\varphi\left(W_{j}\right) \subseteq \mathfrak{g}$ is convex. In the case that $G=C^{\infty}(M, K)$ (for $M$ a compact manifold) or in the case that $G=C(X, K)$ (for $X$ a compact space) and $K$ a Lie group with compact Lie algebra, we may without loss of generality assume that $W_{j}=V$. In fact, in these cases there exists convex Ad-invariant zero neighborhoods in $\mathfrak{g}$ on which the exponential map restricts to a diffeomorphism. This yields an equivariant chart $\varphi$ with respect to the conjugation action on $G$ and the adjoint action on $\mathfrak{g}$.

Having fixed these choices we set

$$
V_{i, j}:=\left\{(g, h) \in G \times G: i^{-1} g \in W_{j}, j^{-1} h \in V,(i j)^{-1} g h \in V\right\} .
$$

Since $(g, h) \in V_{g, h}$, we clearly have an open cover of $G \times G$ and because the indexing set is $G \times G$, we have canonical maps $p_{1}, p_{2}, p_{3}: G \times G \rightarrow G$ satisfying $p_{a}\left(V_{i, j}\right) \subseteq V_{p_{a}(i, j)}$ for $a=1,2,3$.

Lemma 3.4. Let $C_{p w}^{\infty}(\Sigma, G)$ denote the space of piece-wise smooth maps from the simplicial complex $\Sigma$ in Figure 3 to $G$ (cf. Remark A.7). On $V_{i, j}$ there exists a smooth map $\alpha_{i, j}: V_{i, j} \rightarrow C_{p w}^{\infty}(\Sigma, G)$ such that

$$
\partial_{\text {sing }} \alpha_{i, j}(g, h)=\widetilde{\alpha}(i)+i . \widetilde{\alpha}\left(i^{-1} g\right)+g . \widetilde{\alpha}(j)+g j . \widetilde{\alpha}\left(j^{-1} h\right)-i j . \widetilde{\alpha}\left((i j)^{-1} g h\right)-\widetilde{\alpha}(i j)
$$

(see Figure 1).

Proof. We first observe that

$$
V_{i, j} \times[0,1]^{2} \ni((g, h),(s, t)) \mapsto i \cdot \widetilde{\alpha}\left(i^{-1} g\right)(s) \cdot \widetilde{\alpha}(j)(1-t) \in G
$$

defines a smooth map (since $i^{-1} g \in V$ if $\left.(g, h) \in V_{i, j}\right)$ and thus a smooth map $\lambda_{i, j}: V_{i, j} \rightarrow C^{\infty}\left([0,1]^{2}, G\right)$. Moreover, we may choose an orientation on $[0,1]^{2}$ such that $\left.\lambda_{i, j}(g, h)\right|_{\partial[0,1]^{2}}$ is the piecewise smooth path

$$
i . \widetilde{\alpha}\left(i^{-1} g\right) * g . \widetilde{\alpha}(j) * \overline{i . \widetilde{\alpha}\left(i^{-1} g\right) \cdot j} * \overline{i . \widetilde{\alpha}(j)}
$$

(here, $*$ means concatenation of paths and ${ }^{-}$means orientation reversion). Choosing an appropriate triangulation of $[0,1]^{2}$ then gives a map $\lambda_{i, j}: V_{i, j} \rightarrow C_{p w}^{\infty}(\Sigma, G)$ with

$$
\partial_{\text {sing }} \lambda_{i, j}(g, h)=i . \widetilde{\alpha}\left(i^{-1} g\right)+g . \widetilde{\alpha}(j)-i . \widetilde{\alpha}\left(i^{-1} g\right) \cdot j-i . \widetilde{\alpha}(j)
$$

Next, we consider the map

$$
V_{i, j} \times[0,1]^{2} \ni((g, h),(s, t)) \mapsto i j . \widetilde{\alpha}\left(\left(j^{-1} \cdot \widetilde{\alpha}\left(i^{-1} g\right)(s) \cdot j\right)\right)(t) \in G .
$$


Since $\varphi\left(W_{j}\right) \subseteq \mathfrak{g}$ is convex and since $i^{-1} g \in W_{j} \subseteq V$ it follows from the construction of $\widetilde{\alpha}\left(i^{-1} g\right)$ in (3.2) that $j^{-1} \widetilde{\alpha}\left(i^{-1} g\right)(s) j \in j^{-1} W_{j} j \subseteq V$ for all $s \in[0,1]$ and thus (3.11) defines a smooth function. This in turn restricts to a piecewise smooth function $V_{i, j} \rightarrow C^{\infty}([0,1], G)$ on the boundary $\partial[0,1]^{2}$,

$$
i . \widetilde{\alpha}\left(i^{-1} g\right) \cdot j * \overline{i j . \widetilde{\alpha}\left((i j)^{-1} g j\right)} * \overline{c_{i j}} * c_{i j}
$$

(where $c_{i j}$ denotes the constant path at $i j \in G$ ). Choosing the same triangulation of $[0,1]^{2}$ as above this yields a smooth map $\mu_{i, j}: V_{i, j} \rightarrow C_{p w}^{\infty}(\Sigma, G)$ with

$$
\partial_{\text {sing }} \mu_{i, j}(g, h)=i . \widetilde{\alpha}\left(i^{-1} g\right) \cdot j-i j \cdot \widetilde{\alpha}\left((i j)^{-1} g j\right) \text {. }
$$

Since $\left.\widetilde{\beta}\right|_{V \times V}$ is smooth, it follows that

$$
\nu_{i, j}: V_{i, j} \rightarrow C_{p w}^{\infty}(\Sigma, G), \quad(g, h) \mapsto i j . \widetilde{\beta}\left((i j)^{-1} g j, j^{-1} h\right)
$$

is smooth with

$$
\left.\partial_{\text {sing }} \nu_{i, j}(g, h)=i j . \widetilde{\alpha}\left((i j)^{-1} g j\right)+g j . \widetilde{\alpha}\left(j^{-1} h\right)-i j \cdot \widetilde{\alpha}\left((i j)^{-1} g h\right)\right)
$$

All together, we have that $\widetilde{\alpha}(i, j):=\widetilde{\beta}(i, j)+\lambda_{i, j}+\mu_{i, j}+\nu_{i, j}$ has the desired properties (where we interpret $\widetilde{\beta}(i, j) \in C_{p w}^{\infty}(\Sigma, G)$ as a constant map).

Lemma 3.5. If $\alpha_{i, j}: V_{i, j} \rightarrow C_{p w}^{\infty}(\Sigma, G)$ is smooth and satisfies (3.10), then

$$
\begin{aligned}
\Phi_{(i, j),\left(i^{\prime}, j^{\prime}\right)}: V_{i, j} \cap V_{i^{\prime}, j^{\prime}} \rightarrow\left\langle C_{p w}^{\infty}(\Sigma, G)\right\rangle_{\mathbb{Z}}, \quad(g, h) & \mapsto \alpha_{i, j}(g, h)-\alpha_{i^{\prime}, j^{\prime}}(g, h) \\
& -\widetilde{\beta}\left(i, i^{-1} i^{\prime}\right)+i \cdot \widetilde{\beta}\left(i^{-1} i^{\prime}, i^{\prime-1} g\right) \\
& -g \cdot \widetilde{\beta}\left(j, j^{-1} j^{\prime}\right)+g j \cdot \widetilde{\beta}\left(j^{-1} j^{\prime}, j^{\prime-1} h\right) \\
& +\widetilde{\beta}\left(i j,(i j)^{-1} i^{\prime} j^{\prime}\right)-i j \cdot \widetilde{\beta}\left((i j)^{-1} i^{\prime} j^{\prime},\left(i^{\prime} j^{\prime}\right)^{-1} g h\right)
\end{aligned}
$$

(see Figure 2) actually takes values in the singular 2-cycles $Z_{2}(G)$ and determines a smooth (aka locally constant) map to $H_{2}(G) \cong \pi_{2}(G)$. We denote this map $V_{i, j} \cap V_{i^{\prime}, j^{\prime}} \rightarrow \pi_{2}(G)$ also by $\Phi_{(i, j),\left(i^{\prime}, j^{\prime}\right)}$ for simplicity.

Proof. The smoothness of $\Phi_{(i, j),\left(i^{\prime}, j^{\prime}\right)}$ follows from the smoothness of $\alpha_{i, j},\left.\beta\right|_{U \times U}$ and from the fact that $i^{-1} i^{\prime} \in V^{2} \subseteq U$ if $V_{i, j} \cap V_{i^{\prime}, j^{\prime}} \neq \emptyset$. Now (3.1) and (3.10) imply

$$
\begin{aligned}
& \partial_{\operatorname{sing}} \Phi_{(i, j),\left(i^{\prime}, j^{\prime}\right)}(g, h) \\
= & \widetilde{\alpha}(i)+i \cdot \widetilde{\alpha}\left(i^{-1} g\right)+g \cdot \widetilde{\alpha}(j)+g j \cdot \widetilde{\alpha}\left(j^{-1} h\right)-i j \cdot \widetilde{\alpha}\left((i j)^{-1} g h\right)-\widetilde{\alpha}(i j) \\
- & \widetilde{\alpha}\left(i^{\prime}\right)-i^{\prime} \cdot \widetilde{\alpha}\left(i^{\prime-1} g\right)-g \cdot \widetilde{\alpha}\left(j^{\prime}\right)-g j^{\prime} \cdot \widetilde{\alpha}\left(j^{\prime-1} h\right)+i^{\prime} j^{\prime} \cdot \widetilde{\alpha}\left(\left(i^{\prime} j^{\prime}\right)^{-1} g h\right)+\widetilde{\alpha}\left(i^{\prime} j^{\prime}\right) \\
- & \left(\widetilde{\alpha}(i)+i \cdot \widetilde{\alpha}\left(i^{-1} i^{\prime}\right)-\widetilde{\alpha}\left(i^{\prime}\right)\right)+i \cdot\left(\widetilde{\alpha}\left(i^{-1} i^{\prime}\right)+i^{-1} i^{\prime} \cdot \widetilde{\alpha}\left(i^{\prime-1} g\right)-\widetilde{\alpha}\left(i^{-1} g\right)\right) \\
- & . g\left(\widetilde{\alpha}(j)+j \cdot \widetilde{\alpha}\left(j^{-1} j^{\prime}\right)-\widetilde{\alpha}\left(j^{\prime}\right)\right)+g j \cdot\left(\widetilde{\alpha}\left(j^{-1} j^{\prime}\right)+j^{-1} j^{\prime} \cdot \widetilde{\alpha}\left(j^{\prime-1} h\right)-\widetilde{\alpha}\left(j^{-1} h\right)\right) \\
+ & \left(\widetilde{\alpha}(i j)+i j \cdot \widetilde{\alpha}\left((i j)^{-1} i^{\prime} j^{\prime}\right)-\widetilde{\alpha}\left(i^{\prime} j^{\prime}\right)\right) \\
- & i j \cdot\left(\widetilde{\alpha}\left((i j)^{-1} i^{\prime} j^{\prime}\right)+(i j)^{-1} i^{\prime} j^{\prime} \cdot \widetilde{\alpha}\left(i^{\prime} j^{\prime-1} g h\right)-\widetilde{\alpha}\left((i j)^{-1} g h\right)\right)=0,
\end{aligned}
$$

and the claim follows. 
The maps $\alpha_{i, j}: V_{i, j} \rightarrow C_{p w}^{\infty}(\Sigma, G)$ (composed with the integration map $C_{p w}^{\infty}(\Sigma, G) \rightarrow \mathfrak{z}$ ) and $\Phi_{(i, j),\left(i^{\prime}, j^{\prime}\right)}: V_{i, j} \cap V_{i^{\prime}, j^{\prime}} \rightarrow \pi_{2}(G)$ will yield multiplication morphisms on the groupoid $\mathcal{G}_{\omega}$. What we need in addition in order to turn $\mathcal{G}_{\omega}$ into a Lie 2-group is a 2-morphism yielding the associator. This will be furnished by the next construction. For this, we note that we have an open cover

$$
V_{i, j, l}:=\left\{(g, h, k) \in G^{\times 3}:(g, h) \in V_{i, j},(g, h k) \in V_{i, j l},(g h, k) \in V_{i j, l},(h, k) \in V_{j, l}\right\} .
$$

of $G \times G \times G$ and the canonical maps $p_{a}: G^{\times 3} \rightarrow G^{\times 2}$ (for $a=1,2,3,4$ ) satisfy $p_{a}\left(V_{i, j, l}\right) \subseteq V_{p(i, j, l)}$.

Lemma 3.6. If $\alpha_{i, j}: V_{i, j} \rightarrow C_{p w}^{\infty}(\Sigma, G)$ is smooth and satisfies (3.10) then for each $(i, j, l) \in G^{3}$, the map

$\Theta_{i, j, l}: V_{i, j, l} \rightarrow\left\langle C_{p w}^{\infty}(\Sigma, G)\right\rangle_{\mathbb{Z}}, \quad(g, h, k) \mapsto-g . \alpha_{j, l}(h, k)+\alpha_{i j, l}(g h, k)-\alpha_{i, j l}(g, h k)+\alpha_{i, j}(g, h)$

takes values in the singular 2-chains $Z_{2}(G)$ and determines a smooth (aka constant) map to $H_{2}(G) \cong \pi_{2}(G)$.

Proof. From (3.10) we get

$$
\begin{aligned}
\partial_{\text {sing }} \Theta_{i, j l}(g, h, k)= & g \cdot\left(\widetilde{\alpha}(j)+j \cdot \widetilde{\alpha}\left(j^{-1} h\right)+h . \widetilde{\alpha}(l)+h l . \widetilde{\alpha}\left(l^{-1} k\right)-j l . \widetilde{\alpha}\left((j l)^{-1} h k\right)-\widetilde{\alpha}(j l)\right) \\
- & \widetilde{\alpha}(i j)+i j . \widetilde{\alpha}\left((i j)^{-1} g h\right)+g h . \widetilde{\alpha}(l)+g h l . \widetilde{\alpha}\left(l^{-1} k\right)-i j l . \widetilde{\alpha}\left((i j l)^{-1} g h k\right)-\widetilde{\alpha}(i j l) \\
& \left(\widetilde{\alpha}(i)+i . \widetilde{\alpha}\left(i^{-1} g\right)+g . \widetilde{\alpha}(j l)+g j l . \widetilde{\alpha}\left((j l)^{-1} h k\right)-i j l . \widetilde{\alpha}\left((i j l)^{-1} g h k\right)-\widetilde{\alpha}(i j l)\right) \\
- & \widetilde{\alpha}(i)+i . \widetilde{\alpha}\left(i^{-1} g\right)+g . \widetilde{\alpha}(j)+g j . \widetilde{\alpha}\left(j^{-1} h\right)-i j . \widetilde{\alpha}\left((i j)^{-1} g h\right)-\widetilde{\alpha}(i j)=0 .
\end{aligned}
$$

Then the claim follows from and the fact that $\alpha_{i, j}$ is smooth on $V_{i, j}$.

We now set

$$
F_{i, j}:=\operatorname{per}_{\omega} \circ \alpha_{i, j}\left(:=\int_{\alpha_{i, j}} \omega^{l}\right),
$$

which is a smooth map from $V_{i, j}$ to $\mathfrak{z}$ by the assumption on $\alpha_{i, j}$.

Lemma 3.7. There is a simplicial cover of $\mathbf{B} G$ • with $\mathcal{U}^{(1)}=\left\{V_{i}\right\}, \mathcal{U}^{(2)}=\left\{V_{i, j}\right\}$ and $\mathcal{U}^{(3)}=\left\{V_{i, j, l}\right\}$ with $V_{i}, V_{i, j}$ and $V_{i, j, l}$ defined in (3.6), (3.9) and (3.13) respectively. Moreover

$$
\left(\gamma_{i, j}, \eta_{i, j, l}, F_{i, j},-\Phi_{(i, j),\left(i^{\prime}, j^{\prime}\right)}, \Theta_{i, j, k}\right)
$$

as defined in (3.7), (3.8), (3.15), (3.12) and (3.14) constitutes a differentiable $\pi_{2}(G) \stackrel{\text { per }_{\omega}}{\longrightarrow}$ $\mathfrak{z}$-valued cocycle on $\mathrm{B} G$. with respect to this cover. 
Proof. A simplicial cover of $\mathbf{B} G_{\bullet}$ is induced as follows. As indexing simplicial set we take $I^{\bullet}$ with $I^{(n)}=G^{n}$ with the standard simplicial maps from Example 2.1. Then $\mathcal{U}^{(1)}$, $\mathcal{U}^{(2)}$ and $\mathcal{U}^{(3)}$ are covers of $\mathrm{B} G_{1}, \mathrm{~B} G_{2}$ and $\mathrm{B} G_{3}$ respectively, which are by construction compatible with all simplicial maps. We now define the cover of $\mathrm{B} G_{n}$ inductively from the one of $\mathrm{B} G_{n-1}$ by setting

$$
U_{g_{1}, \ldots, g_{n}}^{(n)}=\bigcap_{k=0}^{n}\left(d_{k}^{n}\right)^{-1}\left(U_{d_{k}^{n}\left(g_{1}, \ldots, g_{n}\right)}^{(n-1)}\right) .
$$

Then $d_{k}^{n}\left(U_{g_{1}, \ldots, g_{n}}^{(n)}\right) \subseteq U_{d_{k}^{n}\left(g_{1}, \ldots, g_{n}\right)}^{(n-1)}$ holds by definition and since $d_{k}^{n} \circ s_{k}^{n}=$ id we have $s_{k}^{n}\left(U_{g_{1}, \ldots, g_{n}}^{(n)}\right) \subseteq U_{s_{k}^{n}\left(g_{1}, \ldots, g_{n}\right)}^{n+1}$.

Plugging in the definitions we obtain immediately

$$
\gamma_{j, l}(x)-\gamma_{i, l}(x)+\gamma_{i, j}(x)+\operatorname{per}_{\omega}\left(\eta_{i, j, l}(x)\right)=0 \quad \text { for } \quad x \in V_{i} \cap V_{j} \cap V_{l} .
$$

Since $\alpha_{i, j}: V_{i, j} \rightarrow C_{p w}^{\infty}(\Sigma, G)$ satisfies (3.10), it follow from this and (3.7) that

$$
\operatorname{per}_{\omega}\left(\Phi_{(i, j),\left(i^{\prime}, j^{\prime}\right)}(g, h)\right)=F_{i, j}(g, h)-F_{i^{\prime}, j^{\prime}}(g, h)-\gamma_{i, i^{\prime}}(g)-\gamma_{j, j^{\prime}}(h)+\gamma_{i j, i^{\prime} j^{\prime}}(g h)
$$

for $(g, h) \in V_{i, j} \cap V_{i^{\prime}, j^{\prime}}$ (see (3.12) and Figure 2 for the definition of $\left.\Phi_{(i, j),\left(i^{\prime}, j^{\prime}\right)}\right)$. Finally, the maps $\Theta_{i, j, l}: V_{i, j, l} \rightarrow \pi_{2}(G)$ satisfy

$$
\operatorname{per}_{\omega}\left(\Theta_{i, j, l}(g, h, k)\right)+F_{j, l}(h, k)-F_{i j, l}(g h, k)+F_{i, j l}(g, h k)-F_{i, j}(g, h)=0
$$

by their very definition (3.14) and $F_{i j}=\operatorname{per}_{\omega} \circ \alpha_{i j}$.

In order to obtain a differentiable cocycle, we have to check some further properties. In fact, we obtain

$$
\eta_{j, k, m}(g)-\eta_{i, l, m}(g)+\eta_{i, j, m}(g)-\eta_{i, j, l}(g)=0
$$

for $g \in V_{i} \cap V_{j} \cap V_{l} \cap V_{m}$ by plugging in the definition of $\eta_{i, j, l}(g)$ from (3.8). To check the compatibility of $\Phi_{(i, j),\left(i^{\prime}, j^{\prime}\right)}$ with $\eta_{i, j, l}$ we observe that

$$
\begin{aligned}
& \Phi_{\left(i^{\prime}, j^{\prime}\right),\left(i^{\prime \prime}, j^{\prime \prime}\right)}(g, h)- \Phi_{(i, j),\left(i^{\prime \prime}, j^{\prime \prime}\right)}(g, h)+\Phi_{(i, j)\left(i^{\prime}, j^{\prime}\right)}(g, h)= \\
& \widetilde{\Theta}\left(i, i^{-1} i^{\prime}, i^{-1} i^{\prime \prime}\right)+\widetilde{\Theta}\left(i^{-1} i^{\prime}, i^{\prime-1} i^{\prime \prime}, i^{\prime \prime-1} g\right) \\
&+\widetilde{\Theta}\left(j, j^{-1} j^{\prime}, j^{-1} j^{\prime \prime}\right)+\widetilde{\Theta}\left(j^{-1} j^{\prime}, j^{\prime-1} j^{\prime \prime}, j^{\prime \prime-1} g\right) \\
&-\widetilde{\Theta}\left(i j,(i j)^{-1} i^{\prime} j^{\prime},\left(i^{\prime} j^{\prime}\right)^{-1} i^{\prime \prime} j^{\prime \prime}\right)-\widetilde{\Theta}\left((i j)^{-1} i^{\prime} j^{\prime},\left(i^{\prime} j^{\prime}\right)^{-1} i^{\prime \prime} j^{\prime \prime},\left(i^{\prime \prime} j^{\prime \prime}\right)^{-1} g\right)= \\
&-\eta_{i, i^{\prime}, i^{\prime \prime}}(g)-\eta_{j, j^{\prime}, j^{\prime \prime}}(h)+\eta_{i j, i^{\prime} j^{\prime}, i^{\prime \prime} j^{\prime \prime}}(g h),
\end{aligned}
$$

where the first equality follows from the definition of $\Phi_{(i, j),\left(i^{\prime}, j^{\prime}\right)}$ in (3.12) and of $\widetilde{\Theta}$ in (3.3) (note that $G$ acts trivially on $\pi_{2}(G)$ ) and the second equality follows from (3.8) and the cocycle identity for $\widetilde{\Theta}$. The compatibility of $\Theta_{i, j, l}$ and $\Phi_{(i, j),\left(i^{\prime}, j^{\prime}\right)}$ in turn reads

$$
\begin{aligned}
& \Theta_{i^{\prime}, j^{\prime}, l^{\prime}}(g, h, k)-\Theta_{i, j, l}(g, h, k)= \\
& \quad g . \Phi_{(j, l),\left(j^{\prime}, l^{\prime}\right)}(h, k)-\Phi_{(i j, l),\left(i^{\prime} j^{\prime}, l^{\prime}\right)}(g h, k)+\Phi_{(i, j l)\left(i^{\prime}, j^{\prime} l^{\prime}\right)}(g, h k)-\Phi_{(i, j),\left(i, j^{\prime}\right)}(g, h),
\end{aligned}
$$


which follows by plugging the definitions of $\Theta_{i, j, l}$ from (3.14) and of $\Phi_{(i, j),\left(i^{\prime}, j^{\prime}\right)}$ from (3.12). Finally, we have to check that $\Theta_{i, j, l}$ is closed with respect to $\delta_{\mathrm{gp}}$

$g . \Theta_{j, l, m}(h, k, o)-\Theta_{i j, l, m}(g h, k, o)+\Theta_{i, j l, m}(g, h k, o)-\Theta_{i, j, l m}(g, h, k o)+\Theta_{i, j, l}(g, h, k)=0$,

which follows from the simple fact that $\Theta_{i, j, k}$ is already the image of $\alpha_{i, j}$ under $\delta_{\mathrm{gp}}$.

\subsection{Dependency on choices}

In this section we shall briefly discuss the dependence of the construction from the previous section on the various choices that we made. If we first fix the Lie algebra 2-cocycle $\omega$, then we are left with

(1.a) the choice of $\widetilde{\alpha}: G \rightarrow C^{\infty}\left(\Delta^{(1)}, G\right)$

(1.b) the choice of $\widetilde{\beta}: G \times G \rightarrow C^{\infty}\left(\Delta^{(2)}, G\right)$

(1.c) the choice of the identity neighborhoods $U, V$

(1.d) the choice of the open cover $\left(V_{i, j}\right)_{(i, j) \in G \times G}$ of $G \times G$

(1.e) the choice of $\alpha_{i, j}: V_{i, j} \rightarrow C_{p w}^{\infty}(\Sigma, G)$.

Those choices were made in a way such that they satisfy

(2.a) $\widetilde{\alpha}$ is smooth on $U$ and $\widetilde{\beta}$ is smooth on $U \times U$

(2.b) $\partial_{\text {sing }} \widetilde{\beta}(g, h)=\widetilde{\alpha}(g)+g \cdot \widetilde{\alpha}(h)-\widetilde{\alpha}(g h)$

(2.c) $V=V^{-1}$ and $V^{2} \subseteq U$

(2.d) $\operatorname{pr}_{2}\left(V_{i, j}\right) \subseteq j \cdot V, V_{i, j} \cdot V_{i, j} \subseteq(i j) \cdot V, \operatorname{pr}_{1}\left(V_{i, j}\right) \subseteq i \cdot V$ and $(i, j) \in V_{i, j}$

(2.e) $\alpha_{i, j}: V_{i, j} \rightarrow C_{p w}^{\infty}(\Sigma, G)$ is smooth and satisfies (3.10), i.e.

$$
\partial_{\text {sing }} \alpha_{i, j}(g, h)=\widetilde{\alpha}(i)+i . \widetilde{\alpha}\left(i^{-1} g\right)+g . \widetilde{\alpha}(j)+g j . \widetilde{\alpha}\left(j^{-1} h\right)-i j \cdot \widetilde{\alpha}\left((i j)^{-1} g h\right)-\widetilde{\alpha}(i j) .
$$

Moreover, we constructed $\widetilde{\alpha}$ and $\widetilde{\beta}$ on an identity neighborhood with the aid of a chart $\varphi$ and $V_{i, j}$ with the aid of open identity neighborhoods $W_{j} \subseteq G$ for each $j \in G$.

Remark 3.8. All the remaining data of the differentiable cocycle $\left(\gamma_{i, j}, \eta_{i, j, l}, F_{i, j},-\Phi_{(i, j),\left(i^{\prime}, j^{\prime}\right)}, \Theta_{i, j, l}\right)$, including the cover of $\mathbf{B} G$ • were constructed from these choices (cf. (3.7), (3.8), (3.15), (3.12), (3.14) and (3.13)). If we have another collection

$$
\widetilde{\alpha}^{\prime}, \widetilde{\beta}^{\prime}, U^{\prime}, V^{\prime}, V_{i, j}^{\prime} \text { and } \alpha_{i, j}^{\prime}
$$


of the data (1.a)-(1.e) satisfying the conditions (2.a)-(2.e), then we obtain another differentiable cocycle $\left(\gamma_{i, j}^{\prime}, \eta_{i, j, l}^{\prime}, F_{i, j}^{\prime}, \Phi_{(i, j),\left(i^{\prime}, j^{\prime}\right)}^{\prime}, \Theta_{i, j, l}^{\prime}\right)$ by the aforementioned construction. We will argue now that these two differentiable cocycles differ by a coboundary. In particular, the choices of the chart $\varphi: P \rightarrow \varphi(P) \subseteq \mathfrak{g}$ and of the $W_{j}$ are also inessential. The coboundary can be constructed from the given data as follows.

Now $\partial_{\text {sing }}\left(\widetilde{\alpha}(g)-\widetilde{\alpha}^{\prime}(g)\right)=0$ (interpreting $\widetilde{\alpha}(g)-\widetilde{\alpha}^{\prime}(g)$ as a singular 1-chain) and since $G$ is assumed to be simply connected, there exists for each $g \in G$ a map $\widetilde{A}(g) \in$ $C\left(\Delta^{(2)}, G\right)$ such that

$$
\partial_{\text {sing }} \widetilde{A}(g)=\widetilde{\alpha}(g)-\widetilde{\alpha}^{\prime}(g) .
$$

Moreover, we may assume without loss of generality that $\widetilde{A}(g) \in C^{\infty}\left(\Delta^{(2)}, G\right)$ and that $g \mapsto \widetilde{A}(g)$ is smooth on some identity neighborhood $U^{\prime \prime} \subseteq U \cap U^{\prime}$. Let $V^{\prime \prime} \subseteq U^{\prime \prime}$ be open with $e \in V^{\prime \prime}=V^{\prime \prime-1} \subseteq V \cap V^{\prime}$. With this, we set

$$
\xi_{i}: V_{i}^{\prime \prime} \rightarrow \mathfrak{z}, \quad g \mapsto \int_{\widetilde{A}(i)} \omega^{l}+\int_{i . \widetilde{A}\left(i^{-1} g\right)} \omega^{l}
$$

with $V_{i}^{\prime \prime}:=i \cdot V^{\prime \prime}$ for $i \in G$,

$$
\begin{aligned}
\rho_{i, j}: V_{i}^{\prime \prime} \cap V_{j}^{\prime \prime} \rightarrow \pi_{2}(G), \quad g \mapsto \widetilde{\beta}\left(i, i^{-1} g\right)-i . \widetilde{\beta}\left(i^{-1} j, j^{-1} g\right)-\left(\widetilde{\beta^{\prime}}\left(i, i^{-1} g\right)-i \cdot \widetilde{\beta}^{\prime}\left(i^{-1} j, j^{-1} g\right)\right) \\
-\left(\widetilde{A}(i)+i . \widetilde{A}\left(i^{-1} g\right)\right)+\widetilde{A}(j)+j . \widetilde{A}\left(j^{-1} g\right)
\end{aligned}
$$

and

$$
\begin{aligned}
\sigma_{i, j}: V_{i, j}^{\prime \prime} \rightarrow \pi_{2}(G), \quad(g, h) \mapsto & \alpha_{i, j}(g, h)-\alpha_{i, j}^{\prime}(g, h)-\left(\widetilde{A}(i)+i . \widetilde{A}\left(i^{-1}\right)\right) \\
& -\left(\widetilde{A}(j)+j . \widetilde{A}\left(j^{-1} g\right)\right)+\widetilde{A}(i j)+i j . \widetilde{A}\left((i j)^{-1} g h\right)
\end{aligned}
$$

with

$$
V_{i, j}^{\prime \prime}:=\left\{(g, h) \in V_{i, j} \cap V_{i, j}^{\prime} \mid g \in i \cdot V^{\prime \prime}, h \in j \cdot V^{\prime \prime}, g h \in i j \cdot V^{\prime \prime}\right\}
$$

for $(i, j) \in G \times G$. One readily checks with (3.1), (3.16) and (3.10) that $\rho_{i, j}(g)$ and $\sigma_{i, j}(g, h)$ are in fact closed singular 2-chains on $G$ and thus define elements of $\pi_{2}(G)$. Moreover, it follows from the smoothness assumptions on $\widetilde{\beta}, \widetilde{\beta}, \widetilde{A}, \alpha_{i, j}$ and $\alpha_{i, j}^{\prime}$ that $\xi_{i}$, $\rho_{i, j}$ and $\sigma_{i, j}$ define smooth maps. Now, a lengthy but straight-forward calculation shows that

$$
\begin{aligned}
\check{\delta}(\xi)+\operatorname{per}_{\omega} \circ \rho & =\gamma-\gamma^{\prime} \\
\delta_{\mathrm{gp}}(\xi)+\operatorname{per}_{\omega} \circ \sigma & =F-F^{\prime} \\
\check{\delta}(\rho) & =\eta-\eta^{\prime} \\
\delta_{\mathrm{gp}}(\rho)+\check{\delta}(\sigma) & =-\left(\Phi-\Phi^{\prime}\right) \\
\delta_{\mathrm{gp}}(\sigma) & =\Theta-\Theta^{\prime}
\end{aligned}
$$


holds on the refinement

$$
\cdots \rightrightarrows\left(V_{i, j, l}^{\prime \prime}\right)_{(i, j, l) \in G \times G \times G} \rightrightarrows\left(V_{i, j}^{\prime \prime}\right)_{(i, j) \in G \times G} \rightrightarrows\left(V_{i}^{\prime \prime}\right)_{i \in G},
$$

where $V_{i, j, l}^{\prime \prime}$ is constructed from $V_{i, j}^{\prime \prime}$ as in (3.13).

Now let us fix all the data in (1.a)-(1.e), and take $\omega$ and $\omega^{\prime}$ representing the same class in $H^{2}(\mathfrak{g}, \mathfrak{z})$, i.e., $\omega(x, y)-\omega^{\prime}(x, y)=b([x, y])$ for $b: \mathfrak{g} \rightarrow \mathfrak{z}$ continuous and linear. This then results in two different differentiable cocycles $\phi=\left(\gamma_{i, j}, \eta_{i, j, l}, F_{i, j}, \Phi_{\left.(i, j), i^{\prime}, j^{\prime}\right)}, \Theta_{i, j, l}\right)$ and $\phi^{\prime}=\left(\gamma_{i, j}^{\prime}, \eta_{i, j, l}^{\prime}, F_{i, j}^{\prime}, \Phi_{(i, j),\left(i^{\prime}, j^{\prime}\right)}^{\prime}, \Theta_{i, j, l}^{\prime}\right)$. To see that they are equivalent we define

$$
\xi_{i}: i \cdot V \rightarrow \mathfrak{z}, \quad g \mapsto \int_{\widetilde{\alpha}(i)} b^{l}+\int_{i . \widetilde{\alpha}\left(i^{-1} g\right)} b^{l},
$$

where $b^{l}$ is the left-invariant 1 -from on $G$ with $b^{l}(e)=b$. Moreover, we set

$$
\rho_{i, j}: i \cdot V \cap j \cdot V \rightarrow \pi_{2}(G), \quad g \mapsto-\widetilde{\Theta}\left(i, i^{-1} j, j^{-1} g\right)
$$

and $\sigma=0$. Then a straight-forward computation shows that $\phi$ and $\phi^{\prime}$ differ by the coboundary $D_{3}(\rho, \xi, \sigma)$. In summary, we thus have the following

Proposition 3.9. The class in $\check{H}^{3}\left(\mathbf{B}_{\bullet}, A \stackrel{\mu}{\rightarrow} B\right)$ of the differentiable cocycle

$$
\left(\gamma_{i, j}, \eta_{i, j, l}, F_{i, j}, \Phi_{(i, j),\left(i^{\prime}, j^{\prime}\right)}, \Theta_{i, j, l}\right)
$$

in $\check{H}^{3}\left(\mathbf{B} G_{\bullet}, A \stackrel{\mu}{\rightarrow} B\right)$ from Lemma 3.7 does not depend on the choices that we made throughout Section 3.2.

\subsection{A bundle-theoretic interpretation of the main construction}

If $\tilde{f}: G \times G \rightarrow Z$ is a locally smooth group cocycle, then we obtain a central extension of $G$ by $Z$ as follows. We endow the set $Z \times G$ with the group structure $\widehat{\mu}_{\tilde{f}}((x, g),(y, h))=$ $(x+y+\widetilde{f}(g, h), g h)$ and denote the resulting group by $Z \times_{\widetilde{f}} G$ or shortly $\widehat{G}_{\tilde{f}}$. This turns

$$
Z \rightarrow \widehat{G}_{\widetilde{f}} \rightarrow G
$$

with the canonical maps into a central extension of groups. Let $\widetilde{f}$ be smooth on $U \times U$ for $U \subseteq G$ an open identity neighborhood and $V \subseteq U$ be open such that $e \in V, V=V^{-1}$ and $V \cdot V \subseteq U$. Since $V$ is open in $G, Z \times V$ generates $\widehat{G}_{\tilde{f}}$ and since $Z \times U$ carries a natural manifold structure, Theorem A.2 yields a Lie group structure on $\widehat{G}_{\tilde{f}}$. Clearly, (3.17) is then an exact sequence of Lie groups an since we have the smooth section $U \ni$ $x \mapsto(0, x) \in Z \times U$ it is a locally trivial principal bundle. 
Lemma 3.10. [Woc10, Proposition 2.3] For $i \in G$ we set $V_{i}:=i \cdot V$. Then the assignment

$$
(\tau \widetilde{f})_{i, j}: V_{i} \cap V_{j} \rightarrow Z, \quad g \mapsto \widetilde{f}\left(i, i^{-1} g\right)-\widetilde{f}\left(j, j^{-1} g\right)
$$

defines a smooth Čech cocycle on the open cover $\left(V_{i}\right)_{i \in G}$ of $G$. The locally trivial principal bundle (3.17) has this cocycle as classifying cocycle.

It will be important for an understanding of the construction in this paper to have a coordinate representation of the multiplication map $\widehat{\mu}_{\widetilde{f}}: \widehat{G}_{\widetilde{f}} \times \widehat{G}_{\widetilde{f}} \rightarrow \widehat{G}_{\widetilde{f}}$ in terms of these bundle coordinates.

Remark 3.11. The multiplication map makes the diagram

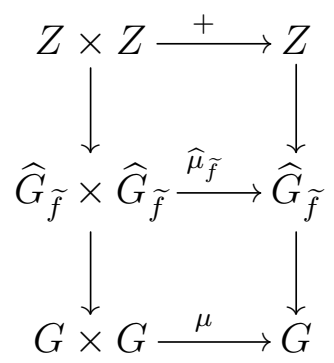

commute, so we obtain a description of $\widehat{\mu}_{\tilde{f}}$ on the open cover $W_{i, j}:=\left\{(g, h) \in V_{i} \times V_{j}\right.$ : $\left.g h \in V_{i j}\right\}$. Since the local trivializations of the bundle $\widehat{G}_{\tilde{f}}$ are given by

$$
Z \times V_{i} \rightarrow \widehat{G}_{\tilde{f}}, \quad(x, g) \mapsto\left(x+\widetilde{f}\left(i, i^{-1} g\right), g\right)
$$

(cf. [Woc10, Proposition 2.3]), one checks directly that the map

$f_{i, j}: W_{i, j} \rightarrow Z, \quad(g, h) \mapsto f_{i, j}(g, h):=\widetilde{f}\left(i, i^{-1} g\right)+\widetilde{f}\left(j, j^{-1} h\right)+\widetilde{f}(g, h)-\widetilde{f}\left(i j,(i j)^{-1} g h\right)$

is the coordinate representation of (the $Z$-component of) the multiplication map $\widehat{\mu}_{\tilde{f}}$. We obtain in this way a morphism

$$
\operatorname{Ext}(G, Z) \cong H_{\mathrm{loc}}^{2}(G, Z) \rightarrow \check{H}^{2}\left(\mathbf{B} G_{\bullet}, 0 \rightarrow Z\right) .
$$

This is an isomorphism, since each class $\phi=\left[\left(\gamma_{i, j}, F_{i}\right)\right]$ in $\check{H}^{2}\left(\mathbf{B} G_{\bullet}, 0 \rightarrow Z\right)$ determines a bundle via its underlying Čech cocycle $\gamma_{i, j}$ and a group structure thereon by the smooth maps $F_{i}$. These assignments are clearly inverse to each other.

From this description it does not follow that $f_{i, j}$ actually is smooth, we only know it because we can put in a whole lot of bundle theory (yielding this expression of the coordinate representation) and group theory (yielding the smoothness of the group multiplication in Theorem A.2). 
The crucial point of the construction in Section 3.2 was that there is an alternative expression for $f_{i, j}$ in the case that $f=q \circ \widetilde{F}$ is the locally smooth cocycle constructed in the case of discrete periods from a Lie algebra cocycle (cf. Proposition 3.3). Indeed, the expression $\int_{\bar{\alpha}_{i, j}} \omega^{l}$ with

$$
\bar{\alpha}_{i, j}(g, h)=\widetilde{\beta}\left(i, i^{-1} g\right)+g \cdot \widetilde{\beta}\left(j, j^{-1} h\right)+\widetilde{\beta}(g, h)-\widetilde{\beta}\left(i j,(i j)^{-1} g h\right)
$$

coincides on $V_{i, j}$ with $\int_{\alpha_{i, j}}$ since it follows immediately from (3.1) and (3.10) that the difference $\alpha_{i, j}(g, h)-\bar{\alpha}_{i, j}(g, h)$ is closed and thus

$$
\int_{\alpha_{i, j}(g, h)-\bar{\alpha}_{i, j}(g, h)} \omega^{l} \in \operatorname{im}\left(\operatorname{per}_{\omega}\right) .
$$

While the smoothness of $f_{i, j}=\int_{\bar{\alpha}_{i, j}} \omega^{l}$ is not immediate from its construction, the smoothness of $\int_{\alpha_{i, j}} \omega^{l}$ is so. This made the construction of the differentiable cocycle in Section 3.2 work.

\section{Lie's Third Theorem}

\subsection{Deriving Lie algebras from étale Lie 2-groups}

We now explain how to associate a Lie algebra to an étale Lie 2-group. Recall from Definition 2.9 that a Lie 2-group is a (weak) group object in the bicategory of smooth stacks (cf. also Appendix B).

Definition 4.1. A Lie groupoid is étale if all its structure maps are local diffeomorphisms. We call a Lie 2-group for which the underlying Lie groupoid is étale an étale Lie 2-group. We denote the full subcategory of étale Lie 2-groups in Lie2 - groups by Lie2 - groupsét.

The next short lemma is the key fact about étale Lie groupoids that will make the construction in the sequel work.

Lemma 4.2. Suppose that $\varphi: \mathcal{G} \rightarrow \mathcal{H}$ is a smooth functor between the Lie groupoids $\mathcal{G}$ and $\mathcal{H}$ and that $\alpha: G_{0} \rightarrow H_{1}$ is a smooth natural transformation with source $\varphi$. If $\mathcal{H}$ is étale and $\alpha(x)=\mathrm{id}\left(\varphi_{0}(x)\right)$ for some $x \in G_{0}$, then $\alpha=\mathrm{id} \circ \varphi_{0}$ on a whole neighborhood of $x$.

Proof. Let $V \subseteq H_{0}$ be open with $\varphi_{0}(x) \in V$ such that id $\left.\right|_{V}$ is a diffeomorphism. Since $\mathbf{s} \circ \mathbf{i d}=\mathrm{id}_{G_{0}}$ we have that $\mathbf{s}$ is the (two-sided) inverse of id on $\operatorname{id}(V)$, and thus it is in particular a right-sided inverse. For $y \in \varphi_{0}^{-1}(V)$ it thus follows from $\mathbf{s}(\alpha(y))=\varphi_{0}(y)$ that $\alpha(y)=\mathbf{i d}(\mathbf{s}(\alpha(y)))=\mathbf{i d}\left(\varphi_{0}(y)\right)$. 
The construction of the Lie algebra associated to the étale Lie 2-group $\mathcal{G}$ is along the lines of [TZ06, Section 5]. The multiplication morphism $m: \mathcal{G} \times \mathcal{G} \rightarrow \mathcal{G}$ (not to be confused with the composition in $\mathcal{G}$ ) is a generalized morphism (see Appendix B) is represented by another Lie groupoid $\mathcal{H}$, a weak equivalence $\mathcal{G} \times \mathcal{G} \stackrel{\Phi}{\leftarrow} \mathcal{H}$ and a smooth functor $\mathcal{H} \stackrel{m}{\rightarrow} \mathcal{G}$. Thus there exists an open neighborhood $U$ of $e \in G_{0}$ (where $e=u_{0}(*)$ ) and a smooth section

$$
\sigma: U \times U \rightarrow H_{0} \Phi_{0} \times_{s_{\mathcal{G}} \times s_{\mathcal{G}}}\left(G_{1} \times G_{1}\right)
$$

of $\left(t_{\mathcal{G}} \times t_{\mathcal{G}}\right) \circ \mathrm{pr}_{2}$. If $\sigma_{1}$ denotes its first component, then $m_{0}: U \times U \rightarrow G_{0},(x, y) \mapsto$ $m_{0}\left(\sigma_{1}(x, y)\right)$ is smooth and represents the restriction of the multiplication in $\mathcal{G}$, restricted to the full sub groupoid $\mathcal{U}:=\left(s_{\mathcal{G}}^{-1}(U) \cap t_{\mathcal{G}}^{-1}(U) \rightrightarrows U\right)$. Note that $m_{1}: G_{1} \times_{U} G_{1} \rightarrow G_{1}$ is uniquely determined by its property to be a smooth functor and the universal property of the pull-back in the definition of weak equivalence.

Now consider the 2-morphism $a: m \circ(m \times$ id $) \Rightarrow m \circ($ id $\times m)$, which is also represented by another Lie groupoid $\mathcal{K}$, a weak equivalence $\mathcal{G} \times \mathcal{G} \times \mathcal{G} \stackrel{\Psi}{\leftarrow} \mathcal{K}$ and a smooth natural transformation $a: K_{0} \rightarrow G_{1}$ between the induced smooth functors $m \circ(m \times$ id) $: \mathcal{K} \rightarrow \mathcal{G}$ and $m \circ($ id $\times m): \mathcal{K} \rightarrow \mathcal{G}$. Inside $U$ we take an open neighborhood $V$ of $e$ such that $m_{0}(V \times V) \subseteq U$ and that there exists a section

$$
\sigma^{\prime}: V \times V \times V \rightarrow K_{0 \Psi_{0}} \times_{s_{\mathcal{G}} \times s_{\mathcal{G}} \times s_{\mathcal{G}}}\left(G_{1} \times G_{1} \times G_{1}\right)
$$

of $\left(t_{\mathcal{G}} \times t_{\mathcal{G}} \times t_{\mathcal{G}}\right) \circ \operatorname{pr}_{2}$. Then $\alpha: V \times V \times V \rightarrow G_{1},(x, y, z) \mapsto a\left(\sigma_{1}(x, y, z)\right)$ defines a smooth natural transformation between $m \circ(m \times$ id $)$ and $m \circ($ id $\times m)$.

Now in general $m(e, e) \neq e$, but we can re-define $m$ to achieve equality here. To this end consider the 2-morphism $\ell: m \circ(u \times$ id $) \Rightarrow$ id, which is represented by another Lie groupoid $\mathcal{L}$, a weak equivalence $\mathcal{G} \stackrel{\Xi}{\leftarrow}$ and a smooth natural transformation $\ell: L_{0} \rightarrow G_{1}$ between the induced smooth functors $m \circ(u \times$ id $): \mathcal{L} \rightarrow \mathcal{G}$ and id: $\mathcal{L} \rightarrow \mathcal{G}$. After possibly shrinking $U$ we may assume that there exists a section $\sigma^{\prime \prime}: U \rightarrow L_{0 \Xi_{0}} \times_{s_{\mathcal{G}}} G_{1}$ of $t_{\mathcal{G}} \circ \mathrm{pr}_{2}$. Then $\lambda: U \rightarrow G_{1}, x \mapsto \ell\left(\sigma^{\prime \prime}(x)\right)$ defines a smooth natural transformation between $m \circ(u \times$ id $)$ and id. With the same procedure we derive $\rho$ from $r: m \circ($ id $\times u) \Rightarrow$ id. Now there exists a neighborhood $U^{\prime}$ of $m(e, e)$ and a section $\bar{\sigma}: U^{\prime} \rightarrow G_{1}$ of $s_{\mathcal{G}}$ with $\bar{\sigma}(m(e, e))=\lambda(e)$. We may assume that $m(U \times U) \subseteq U^{\prime}$ and thus define a smooth natural transformation $U \times U \rightarrow G_{1}$ with source $m$ by $(x, y) \mapsto \bar{\sigma}(m(x, y))$. We now re-define $m$ as the target of this natural transformation. Since $t_{\mathcal{G}}(\bar{\sigma}(m(e, e)))=e$ we thus have that $m(e, e)=e$ holds for the re-defined $m$. If we also use this natural transformation to re-define the other structure morphisms of $\mathcal{G}$, then this endows $\mathcal{U}$ with the structure of a "local Lie 2-group", where "local" means that all morphisms and 2-morphisms defining the group structure are only defined on the full subgroupoid of some neighborhood of $e$.

Since $m(e, e)=e$ holds we have that $\lambda(e)=\rho(e)$ is the identity in $e$ and then also $\alpha(e, e, e)$ due to the coherence of $\ell, r$ and $\alpha$. Now Lemma 4.2 implies that $\alpha(x, y, z)$ is the 
identity of $m(x, m(y, z))=m(m(x, y), z)$ on some neighborhood of $(e, e, e)$, which we may still assume to be $V$. Thus $(U, V, m, e)$ is a local Lie group in the sense of [Nee06, Definition II.10] (the requirement on the existence of inverses follows from requiring $\mathrm{pr}_{1} \times m$ to be invertible with a similar argument as above).

We now have to take care about the choices that we made above. Different choices will lead to a priori different local Lie groups $(U, V, m, e)$ and $\left(U^{\prime}, V^{\prime}, m^{\prime}, e^{\prime}\right)$, and we now argue that they actually agree. We first observe that we can achieve $e=e^{\prime}$ with the same method as above when ensuring $m(e, e)=e$. If we construct $m^{\prime}$ with the aid of a different weak equivalence $\mathcal{G} \times \mathcal{G} \leftarrow \mathcal{H}^{\prime}$, then the functors $m$ and $m^{\prime}$ are smoothly equivalent when restricted to the full subgroupoid

$$
\mathcal{U} \cap \mathcal{U}^{\prime}:=\left(t_{\mathcal{G}}^{-1}\left(U \cap U^{\prime}\right) \cap s_{\mathcal{G}}^{-1}\left(U \cap U^{\prime}\right) \rightrightarrows U \cap U^{\prime}\right) .
$$

Since $m(e, e)=m^{\prime}(e, e)=e$ it follows from the étaleness of $\mathcal{G}$ that this smooth natural transformation is actually the identity on some neighborhood of $e$. We thus see that $m^{\prime}=m$ on some neighborhood of $(e, e)$. Thus the germ of the local group is uniquely determined by $\mathcal{G}$, which in turn determines uniquely a Lie algebra $L(\mathcal{G})$.

Now the same argumentation may also be applied to morphisms to show that the assignment $\mathcal{G} \mapsto L(\mathcal{G})$ actually defines a functor

$$
L: \text { Lie2 - groupsét } \rightarrow \text { LieAlgebras, }
$$

called the Lie functor. It obviously has the property that if we pre-compose it with the fully faithful embedding LieGroups $\rightarrow$ Lie2 - groupsét, given by $G \mapsto \underline{G}$ (see Example 2.11), then it coincides with the ordinary Lie functor $L:$ LieGroups $\rightarrow$ LieAlgebras.

We now observe that the Lie functor is compatible with extensions.

Proposition 4.3. If $A \stackrel{\mu}{\rightarrow} B$ has discrete $A$, then any central extension $[A \stackrel{\mu}{\rightarrow} B] \rightarrow \widehat{G} \rightarrow \underline{G}$ is equivalent to one with étale $\widehat{G}$.

Proof. The Lie groupoid underlying the Lie 2-groups $A \times B \rightrightarrows B$ is étale, and so is the total space of the principal 2-bundle $\mathcal{P}_{(\gamma, \eta)} \rightarrow \underline{G}$, constructed as in Section 2.2.1 from a cocycle $(\gamma, \eta) \in \check{Z}_{\mathcal{U}}^{3}(G, A \stackrel{\mu}{\rightarrow} B)$. The morphism $\mathcal{P}_{(\gamma, \eta)} \rightarrow \widehat{G}$ is a weak equivalence [Woc11b, Proposition 2.19] and thus induces on $\mathcal{P}_{(\gamma, \eta)}$ the structure of a Lie 2-group such that

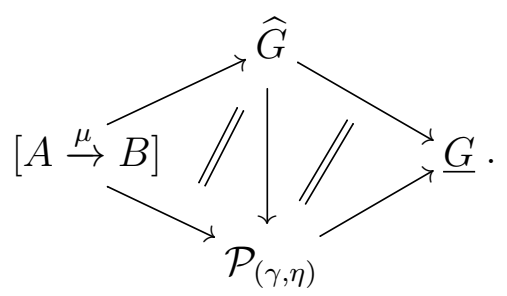

is an equivalence of central extensions. 
Corollary 4.4. If $A \stackrel{\mu}{\rightarrow} B$ has discrete $A$, then the functor (4.1) induces a morphism

$$
D: \operatorname{Ext}(\underline{G},[A \rightarrow B]) \rightarrow \operatorname{Ext}(\mathfrak{g}, L(B)),
$$

given by passing from a central extension $[A \rightarrow B] \rightarrow \widehat{G} \rightarrow \underline{G}$ to an equivalent one with étale $\widehat{G}$ and then applying $L$.

Proof. We first show that if $\widehat{G}$ is étale, then the sequence $L(B) \rightarrow L(\widehat{G}) \rightarrow \mathfrak{g}$ is in fact a central extension of topological Lie algebras. Since the action of $\underline{G}$ on $[A \stackrel{\mu}{\rightarrow} B]$ is trivial for any central extension $[A \stackrel{\mu}{\rightarrow} B] \rightarrow \widehat{G} \rightarrow \underline{G}$ (cf. [SP11, Lemma 84]), we only have to verify that the morphism $L(\widehat{G}) \rightarrow L(G)$ of topological Lie algebras has a continuous linear section. This in turn follows from the local triviality of the principal 2-bundle $\widehat{G} \rightarrow \underline{G}$, since it implies the existence of a smooth local section of the smooth functor $\left(P_{1} \rightrightarrows P_{0}\right) \rightarrow \underline{G}$ for any Lie groupoid $P_{1} \rightrightarrows P_{0}$ representing $\widehat{G}$.

The claim now follows if we can show that for each equivalence of central extensions

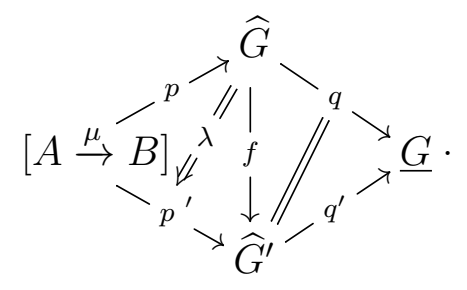

with étale $\widehat{G}$ and $\widehat{G}^{\prime}$ the resulting central extensions of Lie algebras $L(B) \rightarrow L(\widehat{G}) \rightarrow \mathfrak{g}$ and $L(B) \rightarrow L\left(\widehat{G}^{\prime}\right) \rightarrow \mathfrak{g}$ are equivalent. But this readily follows from the fact that $f$ is in particular a morphism of étale Lie 2-groups and $L(f)$ makes the diagram

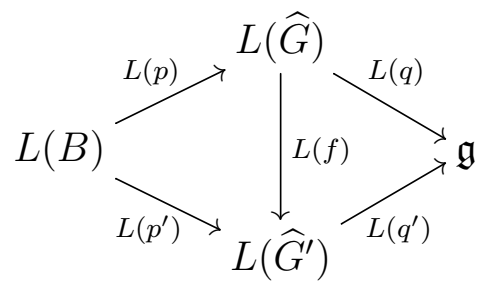

commute.

Remark 4.5. Note that there is also a morphism

$$
D: \check{H}^{2}\left(\mathbf{B} G_{\bullet}, \pi_{2}(G) \stackrel{\operatorname{per}_{\omega}}{\longrightarrow} \mathfrak{z}\right) \rightarrow H_{c}^{2}(\mathfrak{g}, \mathfrak{z})
$$

given by composing the morphism

$$
\check{H}^{2}\left(\mathbf{B} G_{\bullet}, \pi_{2}(G) \stackrel{\text { per }_{\omega}}{\longrightarrow} \mathfrak{z}\right) \rightarrow \operatorname{Ext}\left(\underline{G},\left[\pi_{2}(G) \stackrel{\text { per }_{\omega}}{\longrightarrow} \mathfrak{z}\right]\right)
$$

from Theorem 2.17 with

$$
D: \operatorname{Ext}\left(\underline{G},\left[\pi_{2}(G) \stackrel{\operatorname{per}_{\omega}}{\longrightarrow} \mathfrak{z}\right]\right) \rightarrow \operatorname{Ext}(\mathfrak{g}, \mathfrak{z}) \cong H_{c}^{2}(\mathfrak{g}, \mathfrak{z})
$$


from Corollary 4.4. This is clearly given on the cocycle level by

$$
D F_{i}(x, y):=d^{2} F_{i}(1,1)(x, y)-d^{2} F_{i}(1,1)(y, x)
$$

for some $F_{i}: U_{i}^{(2)} \rightarrow \mathfrak{z}$ with $(1,1) \in U_{i}^{(2)} \subseteq G \times G$ (cf. [Nee02, Lemma 4.6]).

\subsection{Lie's Third Theorem for locally exponential Lie algebras}

Central extensions of the Lie algebra $\mathfrak{g}$ by the abelian Lie algebra $\mathfrak{z}$ are classified by $H_{c}^{2}(\mathfrak{g}, \mathfrak{z})$. We now use this fact and the established integration procedure to give a criterion for a Lie algebra to come from an étale Lie 2-group. For this we first show how $[\omega] \in$ $H_{c}^{2}(\mathfrak{g}, \mathfrak{z})$ and the differentiable cocycle

$$
\phi:=\left(\gamma_{i, j}, \eta_{i, j, l}, F_{i, j}, \Phi_{(i, j),\left(i^{\prime}, j^{\prime}\right)}, \Theta_{i, j, l}\right)
$$

in $\check{H}^{3}\left(\mathbf{B} G_{\bullet}, \pi_{2}(G) \stackrel{\text { per }_{\omega}}{\longrightarrow} \mathfrak{z}\right)$ from Lemma 3.7 are related.

Lemma 4.6. If we apply the Lie functor to the central extension $\left[\pi_{2}(G) \stackrel{\mathrm{per}_{\omega}}{\rightarrow} \mathfrak{z}\right] \rightarrow G_{\phi} \rightarrow \underline{G}$, then this results in a central extension isomorphic to $\mathfrak{z} \oplus_{\omega} \mathfrak{g}$.

Proof. By construction we have to check that the local Lie group associated to $G_{\phi}$ has the Lie algebra $\mathfrak{z} \oplus_{\omega} \mathfrak{g}$. But by Remark 4.5 this is exactly the same argument as [Woc11a, Lemma 1.9] (cf. also [Nee02, Lemma 4.6]).

This immediately implies the following

Theorem 4.7. If $G$ is a simply connected Lie group with Lie algebra $\mathfrak{g}$, $\mathfrak{z}$ is a Mackeycomplete locally convex space and $\omega: \mathfrak{g} \times \mathfrak{g} \rightarrow \mathfrak{z}$ is a continuous Lie algebra cocycle. Then the differentiation homomorphism

$$
D: \operatorname{Ext}\left(\underline{G},\left[\pi_{2}(G) \stackrel{\operatorname{per}_{\omega}}{\longrightarrow} \mathfrak{z}\right]\right) \rightarrow \operatorname{Ext}(\mathfrak{g}, \mathfrak{z}) \cong H_{c}^{2}(\mathfrak{g}, \mathfrak{z})
$$

has $[\omega]$ in its image. Here $\underline{G}$ is the Lie group $G$ viewed as a Lie 2-group (see Example 2.11).

Remark 4.8. We now discuss the relation of the previous theorem to the results from [Nee02]. To this end, consider a morphism $A \stackrel{\mu}{\rightarrow} B$ of abelian Lie groups such that $\operatorname{ker}(\mu) \leq A$ is a closed Lie subgroup and $\operatorname{im} \mu \leq B$ is discrete. Then $\pi:=\operatorname{ker}(\mu)$ and $Z:=B / \operatorname{im}(\mu)$ carry natural Lie group structures and $[\pi \rightarrow 0] \times[0 \rightarrow Z]$ is equivalent to $[A \rightarrow B]$. Moreover, we have an induced sequence

$$
\check{H}^{2}\left(\mathbf{B} G_{\bullet}, \pi \rightarrow 0\right) \stackrel{\chi_{*}}{\rightarrow} \check{H}^{2}\left(\mathbf{B} G_{\bullet}, A \stackrel{\mu}{\rightarrow} B\right) \stackrel{\zeta_{*}}{\rightarrow} \check{H}^{2}\left(\mathbf{B} G_{\bullet}, 0 \rightarrow Z\right) \rightarrow 0
$$

of abelian groups, given by composing cocycles with the canonical morphisms $\chi:(\pi \rightarrow 0) \rightarrow(A \stackrel{\mu}{\rightarrow}$ $B)$ and $\zeta:(A \stackrel{\mu}{\rightarrow} B) \rightarrow(0 \rightarrow Z)$ of chain complexes. 
We claim that (4.2) is exact. It is clear that the sequence is of order two. If $\phi=$ $\left(\gamma_{i, j}, \eta_{i, j, k}, F_{i}, \Phi_{i, j}, \Theta_{i}\right)$ represents a class in $\operatorname{ker}\left(\zeta_{*}\right)$, then there exist $\xi_{i}: U_{i}^{(1)} \rightarrow B$ such that $\gamma_{i, j}+\check{\delta}(\xi)_{i, j}$ and $F_{i}-\delta_{\text {gp }}(\xi)_{i}$ take values in $\operatorname{im}(\mu)^{11}$. We thus may lift (after possibly refining the cover) $\gamma_{i, j}+\check{\delta}(\xi)_{i, j}$ to an $A$-valued cochain $\rho_{i, j}: U_{i}^{(1)} \cap U_{j}^{(1)} \rightarrow A$ and $F_{i}-$ $\delta_{\mathrm{gp}}(\xi)_{i}$ to an $A$-valued cochain $\sigma_{i}: U_{i}^{(2)} \rightarrow A$. Then $\eta^{\prime}:=\eta+\check{\delta}(\rho), \Phi^{\prime}:=\Phi-\delta_{\mathrm{gp}}(\rho)-\check{\delta}(\sigma)$ and $\Theta^{\prime}:=\Theta-\delta_{\mathrm{gp}}(\sigma)$ takes values in $\pi$ :

$$
\begin{aligned}
\mu \circ \eta^{\prime} & =(\mu \circ \eta)+(\mu \circ \check{\delta}(\rho))=(\mu \circ \eta)+\check{\delta}(\gamma)=0 \\
\mu \circ \Phi^{\prime} & =(\mu \circ \Phi)-\delta_{\mathrm{gp}}(\gamma+\check{\delta}(\xi))-\check{\delta}\left(F-\delta_{\mathrm{gp}}(\xi)\right)=(\mu \circ \Phi)-\delta_{\mathrm{gp}}(\gamma)-\check{\delta}(F)=0 \\
\mu \circ \Theta^{\prime} & =\mu \circ \Theta-\delta_{\mathrm{gp}}\left(F-\delta_{\mathrm{gp}}(\xi)\right)=\mu \circ \Theta-\delta_{\mathrm{gp}}(F)=0 .
\end{aligned}
$$

\section{Moreover,}

$$
\left(0, \eta^{\prime}, 0, \Phi^{\prime}, \Theta^{\prime}\right)=\phi+D_{3}(\xi, \rho, \sigma)
$$

is equivalent to $\phi$ and clearly contained in the image of $\chi_{*}$. This implies that (4.2) is exact in $\check{H}^{2}\left(\mathbf{B} G_{\bullet}, A \stackrel{\mu}{\rightarrow} B\right)$. Finally, the surjectivity of $\zeta_{*}$ follows from a lifting argument similar to the previous one (i.e., lift $Z$-valued cocycles to $B$-valued cochains and compensate the failure of these lifts to being cocycles by $A$-valued cocycles).

We now apply (4.2) to $\pi_{2}(G) \stackrel{\text { per }_{\omega}}{\longrightarrow} \mathfrak{z}$ in the case that $\operatorname{per}_{\omega}\left(\pi_{2}(G)\right) \leq \mathfrak{z}$ is discrete. The class of the integrating cocycle

$$
\phi:=\left(\gamma_{i, j}, \eta_{i, j, l}, F_{i, j}, \Phi_{(i, j),\left(i^{\prime}, j^{\prime}\right)}, \Theta_{i, j, l}\right)
$$

in $\check{H}^{3}\left(\mathbf{B} G_{\bullet}, \pi_{2}(G) \stackrel{\text { per }_{\omega}}{\longrightarrow} \mathfrak{z}\right)$ from Lemma 3.7 then gets mapped under $\zeta_{*}$ to a class in

$$
\check{H}^{2}\left(\mathbf{B} G_{\bullet}, 0 \rightarrow Z\right) \cong \operatorname{Ext}(G, Z)
$$

(see Remark 3.11). This is precisely the class of the integrating cocycle from [Nee02].

Remark 4.9. Since we assumed that $G$ is simply connected we have that the map

$$
D: \operatorname{Ext}(\underline{G}, \underline{Z}) \cong \check{H}^{2}\left(\mathbf{B} G_{\bullet}, 0 \rightarrow Z\right) \rightarrow H_{c}^{2}(\mathfrak{g}, \mathfrak{z}) \cong \operatorname{Ext}(\mathfrak{g}, \mathfrak{z})
$$

is injective for each $Z=\mathfrak{z} / \Gamma$ with $\Gamma \leq \mathfrak{z}$ discrete (see [Nee02, Theorem 7.12]). More precisely, the kernel of $D$ coincides with the image of

$$
\operatorname{Hom}\left(\pi_{1}(G), Z\right) \rightarrow \operatorname{Ext}(\underline{G}, \underline{Z}), \quad \varphi \mapsto(Z \times \widetilde{G}) / \pi_{1}(G)
$$

\footnotetext{
${ }^{11}$ Strictly speaking, we would have to choose a refinement of the cover $\left(U_{i}^{(1)}\right)_{i \in I_{1}}$ of $G$ and $\xi_{i}$ might only exist on this refinement. The same applies to each coboundary and each lift throughout the entire construction. Since the construction terminates after finitely many steps we may in the end choose a simplicial common refinement of all covers (cf. Lemma 2.2) that are involved and thus may work throughout with one fixed cover.
} 
(where $\widetilde{G} \rightarrow G$ is the simply connected cover of $G$ and $\pi_{1}(G)$ acts on $Z$ via $\varphi$ ). However, the map

$$
D: \check{H}^{3}\left(\mathbf{B} G_{\bullet}, \pi_{2}(G) \stackrel{\text { per }_{\omega}}{\longrightarrow} \mathfrak{z}\right) \rightarrow H_{c}^{2}(\mathfrak{g}, \mathfrak{z})
$$

is in general not injective. For instance, if $\mathfrak{z}=0$, then the differentiable cocycle

$$
\phi:=\left(0, \eta_{i, j, l}, 0, \Phi_{(i, j),\left(i^{\prime}, j^{\prime}\right)}, \Theta_{i, j, l}\right)
$$

in $\check{H}^{2}\left(\mathbf{B} G_{\bullet}, \pi_{2}(G) \stackrel{0}{\rightarrow} 0\right)$ from Lemma 3.7 is in general non-trivial, since it describes the 2-connected cover of $G$ (see Section 5).

In general, put $\pi:=\operatorname{ker}\left(\operatorname{per}_{\omega}\right)$. Then the canonical morphism $\chi:(\pi \rightarrow 0) \rightarrow\left(\pi_{2}(G) \stackrel{\text { per }_{\omega}}{\longrightarrow}\right.$ $\mathfrak{z})$ of chain complexes gives rise to a morphism

$$
\chi_{*}: \check{H}^{2}\left(\mathbf{B} G_{\bullet}, \pi \rightarrow 0\right) \rightarrow \check{H}^{2}\left(\mathbf{B} G_{\bullet}, \pi_{2}(G) \stackrel{\text { per }_{\omega}}{\longrightarrow} \mathfrak{z}\right),
$$

and we claim that the kernel of $D$ coincides with the subgroup $\chi_{*}\left(\check{H}^{2}\left(\mathbf{B} G_{\bullet}, \pi \rightarrow 0\right)\right)$. This is in general non-trivial, for instance if $\pi_{2}(G)$ is finitely generated. Then $\pi$ is a direct summand in $\pi_{2}(G)$ since $\mathfrak{z}$ is torsion free and thus each generator $a$ is either contained in $\operatorname{ker}(\pi)$ or $\operatorname{per}_{\omega}$ is injective on $\langle a\rangle$. If $\pi$ is a direct summand in $\pi_{2}(G)$, then $\chi_{*}$ is clearly injective.

In order to verify the claim, observe that if $D(\phi)=0$, then we can assume that the $\mathfrak{z}$-valued components of $\phi$ are locally constant. Consequently, $\phi$ can also be considered as a cocycle representing an element in $\check{H}^{2}\left(\mathbf{B} G_{\bullet}, \pi_{2}(G) \stackrel{\text { per }_{\omega}}{\longrightarrow} \mathfrak{z}^{\delta}\right)$, where $\mathfrak{z}^{\delta}$ denotes the abelian Lie group $\mathfrak{z}$ with the discrete topology. From Remark 4.8 we have the short exact sequence

$$
\chi_{*}\left(\check{H}^{2}\left(\mathbf{B} G_{\bullet}, \pi \rightarrow 0\right)\right) \hookrightarrow \check{H}^{2}\left(\mathbf{B} G_{\bullet}, \pi_{2}(G) \stackrel{\text { per }_{\omega}}{\rightarrow} \mathfrak{z}^{\delta}\right) \stackrel{\zeta_{*}}{\rightarrow} \check{H}^{2}\left(\mathbf{B} G_{\bullet}, 0 \rightarrow Z^{\delta}\right)
$$

with $Z^{\delta}:=\mathfrak{z}^{\delta} / \operatorname{per}_{\omega}\left(\pi_{2}(G)\right)$. From covering theory it follows that

$$
\check{H}^{2}\left(\mathbf{B} G_{\bullet}, 0 \rightarrow Z^{\delta}\right) \cong \operatorname{Ext}\left(G, Z^{\delta}\right) \cong \operatorname{Hom}\left(\pi_{1}(G), Z\right)
$$

and this vanishes for $G$ being simply connected. This shows the claim.

This now readily implies our generalization of Lie's Third Theorem.

Theorem 4.10. If $\mathfrak{g}$ is a locally convex locally exponential Lie algebra such that $\mathfrak{z}:=$ $\mathfrak{z}(\mathfrak{g}) \subseteq \mathfrak{g}$ is a complemented and Mackey-complete subspace, then there exists an étale Lie 2-group $G$ with $L(G) \cong \mathfrak{g}$.

Proof. Since $\mathfrak{g}$ is locally exponential, $\mathfrak{g}_{\text {ad }}:=\mathfrak{g} / \mathfrak{z}$ has $G_{\text {ad }} \subseteq \operatorname{Aut}(\mathfrak{g})$ as Lie group with $L\left(G_{\mathrm{ad}}\right)=\mathfrak{g}_{\mathrm{ad}}$ [Nee06, Theorem IV.3.8]. Since $\mathfrak{z}$ is complemented, we have that $\mathfrak{g} \cong$ $\mathfrak{z} \oplus_{\omega_{\text {ad }}} \mathfrak{g}_{\text {ad }}$ for the continuous cocycle $\omega_{\text {ad }}: \mathfrak{g}_{\text {ad }} \times \mathfrak{g}_{\text {ad }} \rightarrow \mathfrak{z}$, determined by $(x, y) \mapsto[x, y]_{1}$, 
where $[x, y]_{1}$ is the $\mathfrak{z}$-component with respect so some chosen topological isomorphism $\mathfrak{g} \cong \mathfrak{z} \times \mathfrak{g}_{\mathrm{ad}}$. Thus we may apply the preceding lemma to the central extension

$$
\mathfrak{z}(\mathfrak{g}) \rightarrow \mathfrak{g} \rightarrow \mathfrak{g}_{\mathrm{ad}},
$$

which shows the claim.

\section{An interpretation in terms of 2-connected covers}

The integration of the Lie algebra cocycle $\omega$ to a locally smooth group cocycle is obstructed by $\pi_{2}(G)$ (or more precisely by $\operatorname{per}_{\omega}\left(\pi_{2}(G)\right)$ ). In any case, the obstruction vanishes if $\pi_{2}(G)$ does (which is in particular the case for finite-dimensional Lie groups). For non-simply connected Lie groups one can always pass to the simply connected cover to resolve obstructions coming from non-trivial fundamental groups, but $\pi_{2}(G)$ cannot be ruled out in a similar fashion for the following reasons.

- The 2-connected "cover" $G^{\sharp} \rightarrow G$ exists as a topological group, but it might not have a Lie group structure.

- Even if $G^{\sharp}$ has a Lie group structure and $G^{\sharp} \rightarrow G$ is a submersion (for instance for certain loop groups or $U\left(\ell^{2}(\mathbb{C})\right) \rightarrow P U\left(\ell^{2}(\mathbb{C})\right)$ ), then $G^{\sharp}$ will in general not have a Lie algebra isomorphic to the one of $G$, since the kernel of $G^{\sharp} \rightarrow G$ cannot be discrete.

However, the central extension $\Pi_{2}(G) \rightarrow \underline{G}$ from Example 2.13 takes over the rôle of the 2-connected cover of $G$. This is made precise by the following theorem. Note that the underlying Lie groupoid of $\Pi_{2}(G)$ determines a simplicial manifold $\mathrm{B}_{2}(G)$. If we now assume that $G$ is metrizable, then each $\mathbf{B} \Pi_{2}(G)_{n}$ is so and thus $\mathbf{B} \Pi_{2}(G)$ • is in particular a simplicial group object in the category of compactly generated Hausdorff spaces. On these objects the (ordinary) geometric realization $\left|\Pi_{2}(G)\right|:=\left|\mathbf{B} \Pi_{2}(G) \bullet\right|$ is particularly wellbehaved. Under these requirements we can now show that the canonical map $\left|\Pi_{2}(G)\right| \stackrel{q}{\rightarrow}$ $G$, induced by ev: $P_{e} G \rightarrow G$, is a 2 -connected cover, i.e., is a fibration, $\pi_{2}\left(\left|\Pi_{2}(G)\right|\right)$ vanishes and $\pi_{i}(q)$ is an isomorphism for $i \neq 2$

Theorem 5.1. If $G$ is a metrizable 1-connected Lie group, then $\left|\Pi_{2}(G)\right| \stackrel{q}{\rightarrow} G$ is a 2connected cover of $G$. Moreover, the multiplication functor of $\Pi_{2}(G)$ induces on $\left|\Pi_{2}(G)\right|$ the structure of a group object in the category of compactly generated Hausdorff spaces and $q$ is a morphism thereof.

Proof. (cf. [BCSS07, Theorem 28]) From [NSW13, Proposition 4.9] it follows that $\left|\Pi_{2}(G)\right| \stackrel{q}{\rightarrow}$ $G$ is a fibration and that the fibre is a $K\left(\pi_{2}(G), 1\right)$. Note that the latter agrees with the geometric realization of

$$
\mathcal{K}:=\operatorname{ker}\left(\Pi_{2}(G) \rightarrow G\right)=(\widetilde{\Omega G} \rtimes \Omega G \rightrightarrows \Omega G) .
$$


Then the same argument as in [BCSS07, Theorem 28] now shows that the claim follows if we can show that for the canonical morphism $\underline{\Omega G} \rightarrow \mathcal{K}$ the induced morphism $\pi_{1}(|\underline{\Omega G}|) \rightarrow \pi_{1}(|\mathcal{K}|)$ is an isomorphism. If we consider the diagram

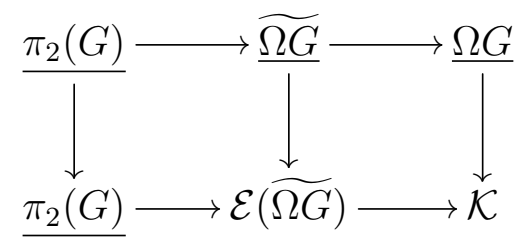

of Lie 2-groups (where $\mathcal{E}(\widetilde{\Omega G})$ is the pair groupoid of $\widetilde{\Omega G}$ ), then the top row is exact by construction and the bottom row is exact by [NSW13, Proposition 4.9]. Since $|\mathcal{E}(\widetilde{\Omega G})|$ is contractible by [Seg68, $\S 3$ ] and [NSW13, Lemma 4.4] and $\pi_{1}(\underline{\Omega G}) \rightarrow \pi_{0}(\underline{G})$ is an isomorphism by construction, the claim follows from the commutativity of

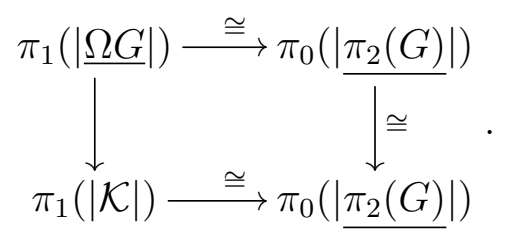

Thus $\Pi_{2}(G) \rightarrow \underline{G}$ may be interpreted as a 2-connected cover. Moreover, there is a smooth version $\Pi_{2}^{\infty}(G)$ of Example 2.13, where one replaces $P_{e} G$ with

$$
P_{e}^{\infty} G:=\left\{\gamma \in C^{\infty}([0,1], G) \mid \gamma(0)=e\right\}
$$

and $\Omega G$ with

$$
\operatorname{ker}(\mathrm{ev}) \cap P_{e}^{\infty} G:=\Omega^{\infty} G:=\left\{\gamma \in C^{\infty}([0,1], G) \mid \gamma(0)=e=\gamma(1)\right\}
$$

Since the inclusions $P_{e}^{\infty} G \hookrightarrow P_{e} G$ and $\Omega^{\infty} G \rightarrow \Omega G$ are homotopy equivalences it follows from [NSW13, Proposition 4.5] that $\left|\Pi_{2}^{\infty}(G)\right| \rightarrow G$ is also a 2-connected cover.

If we apply the construction from Section 3.2 to $\mathfrak{z}=0$ and thus the zero cocycle, then we obtain a differentiable cocycle $\phi_{0}$ and by Proposition 2.19 a central extension

$$
\left[\pi_{2}(G) \rightarrow 0\right] \rightarrow \widehat{G}_{\phi_{0}} \rightarrow \underline{G}
$$

As above, the geometric realization $\left|\widehat{G}_{\phi_{0}}\right| \rightarrow G$ is a 2-connected cover of $G$, but now only as an $A_{\infty}$-space since $\left|\widehat{G}_{\phi_{0}}\right|$ is in general not a group (cf. [SP11, §3.7]) ${ }^{12}$. Moreover, we

\footnotetext{
${ }^{12}$ This can be made precise by constructing an explicit morphism $\widehat{G}_{\phi_{0}} \rightarrow \Pi_{2}^{\infty}(G)$ with the aid of the maps $\widetilde{\alpha}$ and $\widetilde{\beta}$. However, while the construction in Section 3.2 relies on concatenations of paths and triangles, the construction of $\Pi_{2}^{\infty}(G)$ relies on point-wise multiplication. This is the reason why the morphism $\widehat{G}_{\phi_{0}} \rightarrow \Pi_{2}^{\infty}(G)$ would respect the composition (and also the group structure) only up to homotopy. Explicit formulae for this map would exceed its use.
} 
have a canonical morphism $\widehat{G}_{\phi} \rightarrow \widehat{G}_{\phi_{0}}$, induced by forgetting the $\mathfrak{z}$-component in each term. This then gives rise to a central extension

$$
\underline{\mathfrak{z}} \rightarrow \widehat{G}_{\phi} \rightarrow \widehat{G}_{\phi_{0}}
$$

(in the more general setting from [SP11, §3.6]), which can be seen as a central extension

of the 2-connected cover $\widehat{G}_{\phi_{0}}$ of $G$. In this light the construction from Section 3.2 looks as if we have first passed to the 2-connected cover $\widehat{G}_{\phi_{0}}$, where we then have solved the integration problem which is trivial due to the 2-connectedness of $\widehat{G}_{\phi_{0}}$.

\section{A Appendix: Differential calculus on locally convex spaces}

We provide some background material on locally convex Lie groups and their Lie algebras in this appendix. See also [Woc13] for more details on this.

Definition A.1. Let $X$ and $Y$ be locally convex spaces and $U \subseteq X$ be open. Then $f: U \rightarrow Y$ is differentiable or $C^{1}$ if it is continuous, for each $(x, v) \in U \times X$ the differential

$$
d f(x) \cdot v:=\lim _{h \rightarrow 0} \frac{f(x+h v)-f(x)}{h}
$$

exists and if the map $d f: U \times X \rightarrow Y$ is continuous. If $n>1$ we inductively define $f$ to be $C^{n}$ if it is $C^{1}$ and $d f$ is $C^{n-1}$ and to be $C^{\infty}$ or smooth if it is $C^{n}$ for all $n \in \mathbb{N}$. We denote the corresponding spaces of maps by $C^{n}(U, Y)$ and $C^{\infty}(U, Y)$.

A (locally convex) manifold is a topological Hausdorff space (without any further topological requirements) that is locally homeomorphic to open subsets of a locally convex space with smooth chart changes. A (locally convex) Lie group is a group which is a smooth Hausdorff manifold modeled on a locally convex space such that the group operations are smooth. A locally convex Lie algebra is a Lie algebra, whose underlying vector space is locally convex and whose Lie bracket is continuous.

The proof of the following theorem is standard, see for instance [Bou98, Proposition III.1.9.18].

Theorem A.2. Let $G$ be a group $U \subseteq G$ be a subset containing $e$ and let $U$ be endowed with a manifold structure. Moreover, assume that there exists an open neighborhood $V \subseteq U$ of e such that

i) $V^{-1}=V$ and $V \cdot V \subseteq U$,

ii) $V \times V \ni(g, h) \mapsto g h \in U$ is smooth,

iii) $V \ni g \mapsto g^{-1} \in V$ is smooth and 
iv) V generates $G$ as a group.

Then there exists a manifold structure on $G$ such that $V$ is open in $G$ and such that group multiplication and inversion is smooth. Moreover, for each other choice of $V$, satisfying the above conditions, the resulting smooth structures on $G$ coincide.

Definition A.3. Let $G$ be a locally convex Lie group. The group $G$ is said to have an exponential function if for each $x \in \mathfrak{g}$ the initial value problem

$$
\gamma(0)=e, \quad \gamma(t)^{-1} \cdot \gamma^{\prime}(t)=x
$$

has a solution $\gamma_{x} \in C^{\infty}(\mathbb{R}, G)$ and the function

$$
\exp _{G}: \mathfrak{g} \rightarrow G, \quad x \mapsto \gamma_{x}(1)
$$

is smooth. Furthermore, if there exists a zero neighborhood $W \subseteq \mathfrak{g}$ such that $\left.\exp _{G}\right|_{W}$ is a diffeomorphism onto some open identity neighborhood of $G$, then $G$ is said to be locally exponential.

The Fundamental Theorem of Calculus for locally convex spaces (cf. [Glö02a, Theorem 1.5]) yields that a locally convex Lie group $G$ can have at most one exponential function (cf. [Nee06, Lemma II.3.5]).

Typical examples of locally exponential Lie groups are Banach-Lie groups (by the existence of solutions of differential equations and the inverse mapping theorem, cf. [Lan99]) and groups of smooth and continuous mappings from compact manifolds into locally exponential groups ([Glö02b, §3.2], [Woc06]). However, diffeomorphism groups of compact manifolds are never locally exponential (cf. [Nee06, Example II.5.13]) and direct limit Lie groups not always (cf. [Glö05, Remark 4.7]). For a detailed treatment of locally exponential Lie groups and their structure theory we refer to [Nee06, Section IV].

Definition A.4. A locally convex Lie algebra $\mathfrak{g}$ is said to be locally exponential if there exists a circular convex open zero neighborhood $U \subseteq \mathfrak{g}$ and an open subset $D \subseteq U \times U$ on which there exists a smooth map

$$
m_{U}: D \rightarrow U, \quad(x, y) \mapsto x * y
$$

such that $\left(D, U, m_{U}, 0\right)$ is a local Lie group and such that the following holds.

i) For $x \in U$ and $|t|,|s|,|t+s| \leq 1$, we have $(t x, s x) \in D$ with $t x * s x=(t+s) x$.

ii) The second order term in the Taylor expansion of $m_{U}$ in 0 is $b(x, y)=\frac{1}{2}[x, y]$.

As above, one has that all Banach-Lie algebras are locally exponential, as well as all Lie algebras of locally exponential groups (cf. [Nee06, Example IV.2.4]).

Theorem A.5. ([Nee06, Theorem IV.3.8]) Let $\mathfrak{g}$ be a locally exponential Lie algebra. Then the adjoint group $G_{\mathrm{ad}} \leq \operatorname{Aut}(\mathfrak{g})$ carries the structure of a locally exponential Lie group whose Lie algebra is $\mathfrak{g}_{\mathrm{ad}}:=\mathfrak{g} / Z(\mathfrak{g})$. 
Recall that a locally convex space $X$ is said to be convenient or Mackey-complete if each element of $C^{\infty}([0,1], X)$ has an integral in $X$ (cf. [KM97, §I.2]). In particular, complete spaces are convenient [KM97, Theorem I.2.14]. In what follows we silently assume that $\mathfrak{z}$ is a Fréchet space. Then all claims follow from the results of [Woc06]. All what we claim also stays valid if $\mathfrak{z}$ is only assumed to be convenient, the more involved arguments for this case can be found in [KM97].

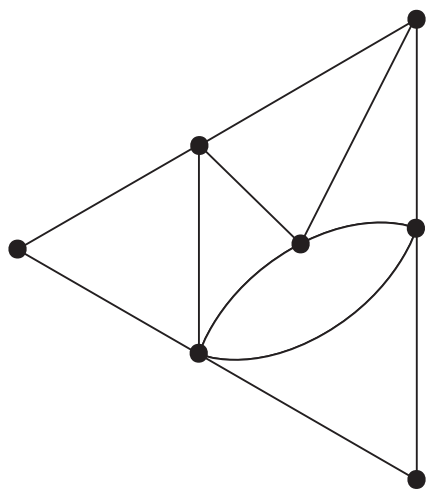

We treat $\Delta^{(n)} \subseteq \mathbb{R}^{n}$ as a manifold with corners as in [Woc06]. Thus a map $f: \Delta^{(n)} \rightarrow G$ is called Figure 3: The triangulation of $\Sigma$ $C^{1}$ if it is differentiable in the interior $\operatorname{int}\left(\Delta^{(n)}\right)$ and in each local chart of $G$ the differentials $(x, v) \mapsto d f(x) . v$ extend continuously to the boundary $\partial\left(\Delta^{(n)}\right)$. It is called $C^{k}$ if it is $C^{1}$ and the differential is $C^{k-1}$ and smooth if it is $C^{k}$ for each $k \in \mathbb{N}$. From this one defines a smooth singular $n$-chain to be a formal sum $f_{1}+\ldots+f_{n}$ of smooth maps $f_{i}: \Delta^{(n)} \rightarrow G$ and denotes by $\mathscr{C}_{n}(G)$ the abelian group of smooth $n$-chains. Since the coface maps $\Delta^{(n-1)} \rightarrow \Delta^{n}$ are smooth, $\partial_{\text {sing }}$ restricts to a boundary operator $\partial_{\text {sing }}: \mathscr{C}_{n}(G) \rightarrow \mathscr{C}_{n-1}(G)$.

If $f:[0,1]^{n} \rightarrow \mathfrak{z}$ is smooth, then

$$
\hat{f}_{n}:[0,1] \rightarrow C^{\infty}\left([0,1]^{n-1}, \mathfrak{z}\right), \quad s \mapsto\left(\left(x_{1}, \ldots, x_{n-1}\right) \mapsto f\left(x_{1}, \ldots, x_{n-1}, s\right)\right)
$$

is smooth. Since $C^{\infty}\left([0,1]^{n-1}, \mathfrak{z}\right)$ is again Fréchet (respectively convenient), it follows from [KM97, Theorem I.2.14] that $\int_{0}^{1} f d x_{n}:=\int_{0}^{1} \hat{f}_{n}$ exists in $\mathfrak{z}$. We thus may define the iterated integral

$$
\int_{[0,1]^{n}} f:=\int_{0}^{1} \ldots\left(\int_{0}^{1} f d x_{n}\right) \ldots d x_{1}
$$

If $\omega$ is a smooth $n$-form on $[0,1]^{n}$, then we set as usual

$$
\int_{[0,1]^{n}} \omega:=\int_{[0,1]^{n}} \omega\left(\partial_{x_{n}}, \ldots, \partial_{x_{1}}\right)
$$

For $q_{n}:[0,1]^{n} \rightarrow \Delta^{(n)}$ a smooth map that restricts to a diffeomorphism on the interior (e.g., take

$$
\left(x_{1}, \ldots, x_{n}\right) \mapsto\left(\left(1-x_{2}\right) \cdots\left(1-x_{n}\right) x_{1}, \ldots,\left(1-x_{n}\right) x_{n-1}, x_{n}\right)
$$

as such a map) and $\omega \in \Omega^{n}\left(\Delta^{(n)}, \mathfrak{z}\right)$, we then define

$$
\int_{\Delta^{(n)}} \omega:=\int_{[0,1]^{n}} q_{n}^{*} \omega .
$$


For $\sigma \in C^{\infty}\left(\Delta^{(n)}, G\right)$ and $\omega \in \Omega^{n}(G, \mathfrak{z})$ we define $\int_{\sigma} \omega:=\int_{\Delta^{(n)}} \sigma^{*} \omega$ and, moreover, for each $\sigma \in \mathscr{C}_{n}(G)$ by additive extension.

Proposition A.6. The map

$$
C^{\infty}\left(\Delta^{(n)}, G\right) \rightarrow \mathfrak{z}, \quad \sigma \mapsto \int_{\sigma} \omega
$$

defined in the previous remark is smooth, where we endow $C^{\infty}\left(\Delta^{(n)}, G\right)$ with the smooth structure from [Woc06].

Proof. Since an atlas on $C^{\infty}\left(\Delta^{(n)}, G\right)$ is given by $\gamma \mapsto \varphi \circ\left(\gamma^{-1} \cdot \gamma^{\prime}\right)$ for $\varphi: U \rightarrow \widetilde{U} \subseteq \mathfrak{g}$ a chart of $G$ and $\gamma^{\prime} \in \gamma \cdot C^{\infty}\left(\Delta^{(n)}, U\right)$, it clearly suffices to show that the map

$$
C^{\infty}\left(\Delta^{(n)}, \mathfrak{g}\right) \rightarrow \mathfrak{z}, \quad \sigma \mapsto \int_{\sigma} \widetilde{\omega}=\int \ldots \int\left(\left(\sigma \circ q_{n}\right)^{*} \widetilde{\omega}\right)\left(\partial_{x_{n}}, \ldots, \partial_{x_{i}}\right) d x_{n} \ldots d x_{1}
$$

is smooth for each $\widetilde{\omega} \in \Omega^{n}\left(\Delta^{(n)}, \mathfrak{g}\right)$. Since $C^{\infty}\left(\Delta^{(n)}, \mathfrak{g}\right) \ni \sigma \mapsto d \sigma \in C^{\infty}\left(T \Delta^{(n)}, \mathfrak{g}\right)$ is linear and continuous (by the definition of the topology on $C^{\infty}\left(\Delta^{(n)}, \mathfrak{g}\right)$ ), and since evaluation is smooth, $d \sigma\left(T q_{n}\left(\partial_{x_{i}}\right)\right) \in C^{\infty}\left([0,1]^{n}, \mathfrak{g}\right)$ depends smoothly on $\sigma$. By the definition of a smooth $n$-form (cf. [Nee06, Definition I.4.1]), $\omega$ is an element of $C^{\infty}(\mathfrak{g} \times$ $\left.\mathfrak{g}^{n}, \mathfrak{z}\right)$ and since push forward is a smooth map on mapping spaces by [Woc06, Corollary 29],

$$
\left.\widetilde{\omega}_{q_{n}(\cdot)}\left(d \sigma\left(T q_{n}\left(\partial_{x_{n}}\right)\right), \ldots, d \sigma\left(T q_{n}\left(\partial_{x_{n}}\right)\right)\right)\right) \in C^{\infty}\left([0,1]^{n}, \mathfrak{z}\right)
$$

depends smoothly on $\sigma$.

Now the integration map, sending a smooth curve to its integral, is continuous and linear, so in particular smooth. This implies the smoothness of (A.1).

Remark A.7. Suppose $\Sigma=\cup_{i \in I} \tau_{i}$ is a simplicial complex, where $\tau_{i}$ are homeomorphic images of $\Delta^{\left(n_{i}\right)}$ for some $n_{i} \in \mathbb{N}_{0}$. We call $\Sigma$ a $p$-complex, if $n_{i}=p$ for all $i \in I$ and finite if $I$ is finite. Unless mentioned otherwise, $\Sigma$ will always refer to the simplicial 2-complex from Figure 3.

A map $f: \Sigma \rightarrow G$ is called piece-wise smooth if it is continuous and $\left.f\right|_{\tau_{i}}$ is smooth for all $i \in I$. The space $C_{p w}^{\infty}(\Sigma, G)$ of piece-wise smooth maps is then also a Lie group with Lie algebra $C_{p w}^{\infty}(\Sigma, \mathfrak{g})$, the construction from [Woc06, $\left.\S 4\right]$ or [Glö02b, §3.2] carries over to this slightly more general situation. The integral of a $\mathfrak{z}$-valued $p$-form $\omega$ over a finite $p$ simplex is defined by additive extension. Since the restriction of piece-wise smooth maps to the simplices is smooth it follows from Proposition A.6 that

$$
C_{p w}^{\infty}(\Sigma, G) \rightarrow \mathfrak{z}, \quad \sigma \mapsto \sum_{i \in I} \int_{\left.\sigma\right|_{\tau_{i}}} \omega
$$

is also smooth. 


\section{B Appendix: Lie groupoids and the submersion Grothendieck pretopology on locally convex manifolds}

Our Lie group(oid)s are based on locally convex manifolds. More precisely they are group(oid) objects in the category of locally convex manifolds endowed with a Grothendieck pretopology that we will explain in this appendix. See also [Woc13] for more details on this.

Definition B.1. A singleton Grothendieck pretopology $\mathcal{T}$ on a category $\mathcal{C}$ is a collection of morphisms, called covers, subject the following three axioms:

1. Isomorphisms are covers.

2. The composition of two covers is a cover.

3. If $U \rightarrow X$ is a cover and $Y \rightarrow X$ is a morphism, then the pull-back $Y \times_{X} U$ is representable, and the natural morphism $Y \times_{X} U \rightarrow Y$ is a cover.

A smooth map $f: M \rightarrow N$ of manifolds is a submersion if for each $x \in M$ there exist charts around $x$ and $f(x)$ such that the coordinate representation of $f$ is a projection. In this case the pull-back of an arbitrary smooth map of a submersion is a manifold and the canonical map $Z \times{ }_{N} M \rightarrow Z$ is again a submersion. Thus the category of such manifolds $\mathcal{C}_{0}$ has a Grothendieck pretopology $\mathcal{T}_{0}$ whose covers are surjective submersions [NSW13, Appendix B].

A groupoid object in $(\mathcal{C}, \mathcal{T})$ is a groupoid $G_{1} \rightrightarrows G_{0}$ such that both $G_{1}, G_{0} \in \mathcal{C}$, all the structure maps are morphisms in $\mathcal{C}$, and the source and target maps $\mathbf{t}, \mathbf{s}$ are covers in $\mathcal{T}$. When $G_{0}$ is the terminal object $*$ in $\mathcal{C}$, a groupoid object is a group object. The theory of groupoids object, stacks, weak equivalence, and generalized morphisms are well known in various categories (see for example [BX11, Met03, MM03, Noo08] and references therein). Such a theory for higher groupoids for general $(\mathcal{C}, \mathcal{T})$ is partially developed in [Zhu09]. Our Lie group(oid) is a group(oid) object in $\left(\mathcal{C}_{0}, \mathcal{T}_{0}\right)$.

We briefly recall here for 1-groupoids to make our paper self-contained the following

Definition B.2. A morphism $f: Z \rightarrow X$ of groupoid objects in $(\mathcal{C}, \mathcal{T})$ is a weak equivalence (see [MM03] if

- the map $Z_{0} \times_{X_{0}} X_{1} \rightarrow X_{0}$ is a cover in $\mathcal{T}$;

- the natural map from $Z_{1}$ to the pull-back $Z_{0} \times_{X_{0}} X_{1} \times_{X_{0}} Z_{0}\left(=X_{1} \times_{X_{0} \times X_{0}} Z_{0} \times Z_{0}\right)$ is an isomorphism.

Definition B.3. A generalized morphism between two groupoid objects $X$ and $Y$ in $(\mathcal{C}, \mathcal{T})$ consists of a span of morphisms $X \stackrel{\sim}{\leftarrow} Z \rightarrow Y$, where the map $Z \stackrel{\sim}{\rightarrow} X$ is a weak equivalence. 
Weak equivalences are in general not invertible as morphisms of groupoid objects. One has to take the symmetric closure to come to an equivalence relation (due to the axioms of the pretopology we only have to take spans of weak equivalences instead of arbitrary zig-zags to achieve this).

Definition B.4. Two groupoid objects $X$ and $Y$ in $(\mathcal{C}, \mathcal{T})$ are Morita equivalent if there is another groupoid $Z$ in $(\mathcal{C}, \mathcal{T})$ and weak equivalences $X \stackrel{\sim}{\leftarrow} Z \stackrel{\sim}{\rightarrow} Y$.

A morphism between two generalized morphisms $X \stackrel{\sim}{\leftarrow} \rightarrow Y$ and $X \stackrel{\sim}{\leftarrow} Z^{\prime} \rightarrow Y$ is given by a third zig-zag $X \stackrel{\sim}{\leftarrow} Z^{\prime \prime} \rightarrow Y$ such that $Z^{\prime \prime}$ also provides a weak equivalence $Z \stackrel{\sim}{\leftarrow} Z^{\prime \prime} \stackrel{\sim}{\rightarrow} Z^{\prime}$ and all triangle diagrams commute up to 2-morphisms of groupoid objects:

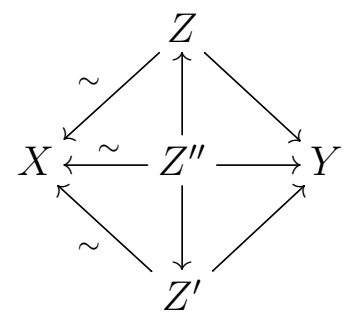

The bicategory of Lie groupoids we use in this article has Lie groupoids in $\left(\mathcal{C}_{0}, \mathcal{T}_{0}\right)$ as objects, generalized morphisms as 1-morphisms, and morphisms between generalized morphisms as 2-morphisms. Morita equivalent Lie groupoids one-to-one corresponds presentable stacks in various categories with Grothendieck pretopologies (see for example [BX11, Met03, Noo08, Pro96]). The same technique will apply here for our case of $\left(\mathcal{C}_{0}, \mathcal{T}_{0}\right)$. Thus our Lie 2-group is in some sense also a stacky Lie group in the sense of [Blo08].

Acknowledgments. The authors would like to thank the referees of this paper for helpful suggestions that lead to several improvements.

\section{References}

[Bar06] Bartels, T. Higher gauge theory I: 2-Bundles 2006. arXiv:math / 0410328

[BCSS07] Baez, J. C., Crans, A. S., Stevenson, D. and Schreiber, U. From loop groups to 2-groups. Homology, Homotopy Appl. 9 (2007)(2):101-135. arXiv:math / 0504123

[BL04] Baez, J. C. and Lauda, A. D. Higher-dimensional algebra. V. 2-groups. Theory Appl. Categ. 12 (2004):423-491 (electronic). arXiv:math. QA/ 0307200

[Blo08] Blohmann, C. Stacky Lie groups. Int. Math. Res. Not. IMRN (2008):Art. ID rnn 082, 51

[Bou98] Bourbaki, N. Lie groups and Lie algebras. Chapters 1-3. Elements of Mathematics (Berlin) (Springer-Verlag, Berlin, 1998). Translated from the French, Reprint of the 1989 English translation

[Bre90] Breen, L. Bitorseurs et cohomologie non abélienne. In The Grothendieck Festschrift, Vol. I, Progr. Math., vol. 86, pp. 401-476 (Birkhäuser Boston, Boston, MA, 1990) 
[Bre94] Breen, L. On the classification of 2-gerbes and 2-stacks. Astérisque 225 (1994):160

[Bry93] Brylinski, J.-L. Loop spaces, characteristic classes and geometric quantization, Progress in Mathematics, vol. 107 (Birkhäuser Boston Inc., Boston, MA, 1993)

[BS76] Brown, R. and Spencer, C. B. G-groupoids, crossed modules and the fundamental groupoid of a topological group. Nederl. Akad. Wetensch. Proc. Ser. A 79=Indag. Math. 38 (1976)(4):296302

[BS07] Baez, J. C. and Schreiber, U. Higher gauge theory. In Categories in algebra, geometry and mathematical physics, Contemp. Math., vol. 431, pp. 7-30 (Amer. Math. Soc., Providence, RI, 2007). (math/0504123), arXiv:math/0511710

[BX11] Behrend, K. and Xu, P. Differentiable stacks and gerbes. J. Symplectic Geom. 9 (2011)(3):285-341. URL http://projecteuclid.org/getRecord?id=euclid. jsg/1310388899. arXiv:math/0605694

[CF03] Crainic, M. and Fernandes, R. L. Integrability of Lie brackets. Ann. of Math. (2) 157 (2003)(2):575-620. doi:10.4007/annals.2003.157.575

[Con03] Conrad, B. Cohomological descent. preprint 2003. URL http://math. stanford. edu/ $\sim \mathrm{conrad/}$

[Ded60] Dedecker, P. Sur la cohomologie non abélienne. I. Canad. J. Math. 12 (1960):231-251

[Del74] Deligne, P. Théorie de Hodge. III. Inst. Hautes Études Sci. Publ. Math. 44 (1974):5-77

[DL66] Douady, A. and Lazard, M. Espaces fibrés en algèbres de Lie et en groupes. Invent. Math. 1 (1966):133-151

[Dus79] Duskin, J. Higher-dimensional torsors and the cohomology of topoi: the abelian theory. In Applications of sheaves (Proc. Res. Sympos. Appl. Sheaf Theory to Logic, Algebra and Anal., Univ. Durham, Durham, 1977), Lecture Notes in Math., vol. 753, pp. 255-279 (Springer, Berlin, 1979)

[EK64] van Est, W. T. and Korthagen, T. J. Non-enlargible Lie algebras. Nederl. Akad. Wetensch. Proc. Ser. A 67=Indag. Math. 26 (1964):15-31

[Est58] van Est, W. T. A group theoretic interpretation of area in the elementary geometries. Simon Stevin 32 (1958):29-38

[Est62a] van Est, W. T. Local and global groups. I. Nederl. Akad. Wetensch. Proc. Ser. A $65=$ Indag. Math. 24 (1962):391-408

[Est62b] van Est, W. T. Local and global groups. II. Nederl. Akad. Wetensch. Proc. Ser. A $65=$ Indag. Math. 24 (1962):409-425

[FB02] Forrester-Barker, M. Group Objects and Internal Categories 2002. arXiv: math/ 0212065

[FHRZ08] Felder, G., Henriques, A., Rossi, C. A. and Zhu, C. A gerbe for the elliptic gamma function. Duke Math. J. 141 (2008)(1):1-74. doi : $10.1215 /$ S $0012-7094-08-14111-0$

[Fri82] Friedlander, E. M. Étale homotopy of simplicial schemes, Annals of Mathematics Studies, vol. 104 (Princeton University Press, Princeton, N.J., 1982)

[Get09] Getzler, E. Lie theory for nilpotent $L_{\infty}$-algebras. Ann. of Math. (2) 170 (2009)(1):271-301. doi:10.4007/annals.2009.170.271

[Gir71] Giraud, J. Cohomologie non abélienne (Springer-Verlag, Berlin, 1971). Die Grundlehren der mathematischen Wissenschaften, Band 179

[GJ99] Goerss, P. G. and Jardine, J. F. Simplicial homotopy theory, Progress in Mathematics, vol. 174 (Birkhäuser Verlag, Basel, 1999) 
[Gle82] Glenn, P. G. Realization of cohomology classes in arbitrary exact categories. J. Pure Appl. Algebra 25 (1982)(1):33-105. doi:10.1016/0022-4049(82) 90094-9

[Glö02a] Glöckner, H. Infinite-dimensional Lie groups without completeness restrictions. In Geometry and Analysis on Finite- and Infinite-Dimensional Lie Groups (Bedlewo, 2000), Banach Center Publ., vol. 55, pp. 43-59 (Polish Acad. Sci., Warsaw, 2002)

[Glö02b] Glöckner, H. Lie group structures on quotient groups and universal complexifications for infinite-dimensional Lie groups. J. Funct. Anal. 194 (2002)(2):347-409. doi:10.1006/jfan.2002.3942

[Glö05] Glöckner, H. Fundamentals of direct limit Lie theory. Compos. Math. 141 (2005)(6):15511577

[GN14] Glöckner, H. and Neeb, K.-H. Infinite-dimensional Lie groups (Springer-Verlag, 2014)

[Gom05] Gomi, K. Equivariant smooth Deligne cohomology. Osaka J. Math. 42 (2005)(2):309-337. URL http://projecteuclid.org/getRecord?id=euclid.ojm/1153494380

[Hen08] Henriques, A. Integrating $L_{\infty}$-algebras. Compos. Math. 144 (2008)(4):1017-1045. arXiv:math/0603563

[KM97] Kriegl, A. and Michor, P. W. The Convenient Setting of Global Analysis, Mathematical Surveys and Monographs, vol. 53 (American Mathematical Society, Providence, RI, 1997)

[Lan99] Lang, S. Fundamentals of differential geometry, Graduate Texts in Mathematics, vol. 191 (Springer-Verlag, New York, 1999)

[LBv11] Li-Bland, D. and Ševera, P. Integration of Exact Courant Algebroids 2011. arXiv:1101.3996

[Met03] Metzler, D. Topological and Smooth Stacks 2003. arXiv:math/0306176

[MM03] Moerdijk, I. and Mrčun, J. Introduction to foliations and Lie groupoids, Cambridge Studies in Advanced Mathematics, vol. 91 (Cambridge University Press, Cambridge, 2003)

[MT10] Mehta, R. A. and Tang, X. From double Lie groupoids to local Lie 2-groupoids. Bulletin of the Brazilian Mathematical Society 42 (2011), no. 4, 651-681 2010. doi:10.1007/s00574-011-0033-4.arXiv:1012.4103

[Nee02] Neeb, K.-H. Central extensions of infinite-dimensional Lie groups. Ann. Inst. Fourier (Grenoble) 52 (2002)(5):1365-1442. URL http://annalif.ujf-grenoble.fr/Vol52/ E5253/E5253.html

[Nee06] Neeb, K.-H. Towards a Lie theory of locally convex groups. Jpn. J. Math. 1 (2006)(2):291-468

[Noo08] Noohi, B. Notes on 2-groupoids, 2-groups and crossed-modules. Homotopy, Homology, and Applications, 9, (2007), no. 1,75-106 2008. arXiv:math/ 0512106

[NSW13] Nikolaus, T., Sachse, C. and Wockel, C. A smooth model for the string group. Int. Math. Res. Not. IMRN (2013)(16):3678-3721. doi:10.1093/imrn/rns154. arXiv:1104.4288

[NW11] Nikolaus, T. and Waldorf, K. Four Equivalent Versions of Non-Abelian Gerbes 2011. arXiv:1103.4815

[Por08] Porst, S. S. Strict 2-Groups are Crossed Modules 2008. arXiv:0812.1464

[Pra68] Pradines, J. Troisième théorème de Lie les groupoïdes différentiables. C. R. Acad. Sci. Paris Sér. A-B 267 (1968):A21-A23

[Pro96] Pronk, D. A. Etendues and stacks as bicategories of fractions. Compositio Math. 102 (1996)(3):243-303. URL http://www.numdam.org/item?id=CM_1996_102_3_ 243_0 
[Seg68] Segal, G. Classifying spaces and spectral sequences. Inst. Hautes Études Sci. Publ. Math. 34 (1968):105-112

[Smi51a] Smith, P. A. The complex of a group relative to a set of generators. I. Ann. of Math. (2) 54 (1951):371-402

[Smi51b] Smith, P. A. The complex of a group relative to a set of generators. II. Ann. of Math. (2) 54 (1951):403-424

[SP11] Schommer-Pries, C. Central extensions of smooth 2-groups and a finite-dimensional string 2-group. Geom. Topol. 15 (2011):609-676. doi:10.2140/gt.2011.15.609. arXiv:0911.2483

[SZ11] Sheng, Y. and Zhu, C. Higher Extensions of Lie Algebroids and Application to Courant Algebroids 2011. arXiv:1103.5920

[TZ06] Tseng, H.-H. and Zhu, C. Integrating Lie algebroids via stacks. Compos. Math. 142 (2006)(1):251-270

[Wei94] Weibel, C. A. An introduction to homological algebra, Cambridge Studies in Advanced Mathematics, vol. 38 (Cambridge University Press, Cambridge, 1994)

[Woc06] Wockel, C. Smooth extensions and spaces of smooth and holomorphic mappings. J. Geom. Symmetry Phys. 5 (2006):118-126. arXiv: math/ 0511064

[Woc10] Wockel, C. Non-integral central extensions of loop groups. Contemp. Math. 519 (2010):203214. arXiv:0910.1937

[Woc11a] Wockel, C. Categorified central extensions, étale Lie 2-groups and Lie's Third Theorem for locally exponential Lie algebras. Adv. Math. 228 (2011):2218-2257. doi:10.1016/j.aim.2011.07.003.arXiv:0812.1673

[Woc11b] Wockel, C. Principal 2-bundles and their gauge 2-groups. Forum Math. 23 (2011):565-610. doi:10.1515/FORM.2011.020. arXiv:0803.3692

[Woc13] Wockel, C. Infinite-dimensional and higher structures in differential geometry. Lecture notes for a course given at the University of Hamburg 2013. URL http://www. math. uni-hamburg.de/home/wockel/teaching/higher_structures.html

[WW13] Wagemann, F. and Wockel, C. A Cocycle Model for Topological and Lie Group Cohomology. Trans. Amer. Math. Soc. (to appear, 32. pp) (2013). arXiv: 1110.3304

[Zhu09] Zhu, C. n-groupoids and stacky groupoids. Int. Math. Res. Not. IMRN 21 (2009):4087-4141. doi:10.1093/imrn/rnp080. arXiv:0801.2057 